Aus der Abteilung Mund-, Kiefer- und Gesichtschirurgie

(Prof. Dr. med. Dr. med. dent. H. Schliephake)

im Zentrum Zahn-, Mund- und Kieferheilkunde

der Medizinischen Fakultät der Universität Göttingen

\title{
Beurteilung des oberen Luftweges bei Patienten mit schlafbezogenen Atemstörungen im \\ Digitalen Volumentomographen (DVT)
}

\author{
INAUGURAL - DISSERTATION \\ zur Erlangung des Doktorgrades \\ für Zahnheilkunde \\ der Medizinischen Fakultät \\ der Georg-August-Universität Göttingen
}

vorgelegt von

Daniel Markiewicz

aus

Bielefeld

Göttingen 2011 
D e k a n:

I. Berichterstatter:

II. Berichterstatter:

Tag der mündlichen Prüfung:
Prof. Dr. med. C. Frömmel

Prof. Dr. med. Dr. med. dent. W. Engelke Prof. Dr. med. Engelke

10.7.2012 
1. Einleitung 1

1.1. Einführung in das Thema 1

1.2. Schlaf, Schlafarchitektur und Schlafstörungen 2

1.3. Anatomie des orofazialen Systems 4

1.4. Das Schlafapnoe-Syndrom 6

1.4.1. Definition 6

$\begin{array}{ll}\text { 1.4.2. Epidemiologie } & 7\end{array}$

1.4.3. Symptome und Folgen 8

1.4.4. Lokale pathophysiologische Zusammenhänge der Atemwege 10

$\begin{array}{ll}\text { 1.4.5. Diagnostik und Therapie } & 13\end{array}$

1.5. Bildgebendes Verfahren: Digitaler Volumentomograph (DVT) 17

1.6. Ziele der Arbeit 19

2. Material und Methode 20

2.1. Patientenkollektiv 20

$\begin{array}{ll}\text { 2.2. Fragebogenanalyse } & 20\end{array}$

2.3. Radiologische Befunderhebung 21

2.3.1. Gerät und Einstellung 21

$\begin{array}{ll}\text { 2.3.2. Analyse der Datensätze } & 23\end{array}$

2.4. Statistische Auswertung 29

2.5. Ethikkommissionsvotum 29

3. Ergebnisse 30

3.1. Ergebnisse der Patientenbefragung 30

3.2. Deskriptive Darstellung der Messungen 32

3.3. Korrelation von Fragebogenergebnissen und radiologischen 34 Messwerten

3.3.1. Alter 34

3.3.2. Body-Mass-Index (BMI) 35

3.3.3. Epworth Sleepiness Scale (ESS) 36

3.3.4. Lautstärke des Schnarchens 37

3.3.5. Häufigkeit des Schnarchens $\quad 39$ 
3.4. Betrachtung des orofazialen Systemzustandes in Relation zu radiologischen Messwerten

3.5. Fallbeispiele

4. Diskussion 51

4.1. Kritische Betrachtung der angewandten Methode 52

4.1.1. Aspekte der Patientenauswahl 52

4.1.2. Aspekte der Fragebogenauswertung 53

4.1.3. Technische Aspekte des DVT 54

4.1.4. Untersuchungsbedingungen $\quad 59$

4.1.5. Datenerhebung und -auswertung 60

4.2. Bedeutung der Messwerte als klinisch relevante Prognoseparameter 63

4.2.1. Korrelation von Messparametern und OSAS bzw. Schnarchen 63

4.2.2. Orofazialer Systemzustand und OSAS 73

$\begin{array}{ll}\text { 4.3. Schlussfolgerung } & 74\end{array}$

$\begin{array}{ll}\text { 5. Zusammenfassung } & 75\end{array}$

$\begin{array}{ll}\text { 6. Abkürzungsverzeichnis } & 77\end{array}$

$\begin{array}{ll}\text { 7. Anhang: Fragebogen } & 78\end{array}$

$\begin{array}{ll}\text { 8. Literaturverzeichnis } & 79\end{array}$ 


\begin{tabular}{|c|c|c|}
\hline Abb. 1: & S. 5 & Abb. 33: \\
\hline Abb. 2: & S. 18 & Abb. 34: \\
\hline Abb. 3: & S. 22 & Abb. 35: \\
\hline Abb. 4: & S. 24 & Abb. 36: \\
\hline Abb. 5: & S. 25 & \\
\hline Abb. 6: & S. 26 & \\
\hline Abb. 7: & S. 27 & \\
\hline Abb. 8: & S. 28 & \\
\hline Abb. 9: & S. 30 & \\
\hline Abb. 10: & S. 30 & \\
\hline Abb. 11: & S. 31 & \\
\hline Abb. 12: & S. 31 & \\
\hline Abb. 13: & S. 32 & \\
\hline Abb. 14: & S. 41 & \\
\hline Abb. 15: & S. 42 & \\
\hline Abb. 16: & S. 43 & \\
\hline Abb. 17: & S. 44 & \\
\hline Abb. 18: & S. 45 & \\
\hline Abb. 19: & S. 46 & \\
\hline Abb. 20: & S. 46 & \\
\hline Abb. 21: & S. 46 & \\
\hline Abb. 22: & S. 47 & \\
\hline Abb. 23: & S. 47 & \\
\hline Abb. 24: & S. 47 & \\
\hline Abb. 25: & S. 47 & \\
\hline Abb. 26: & S. 47 & \\
\hline Abb. 27: & S. 47 & \\
\hline Abb. 28: & S. 48 & \\
\hline Abb. 29: & S. 48 & \\
\hline Abb. 30: & S. 49 & \\
\hline Abb. 31: & S. 49 & \\
\hline Abb. 32: & S. 49 & \\
\hline
\end{tabular}




\section{Tabellenverzeichnis}

Tab. 1: $\quad$ S. 14

$\begin{array}{ll}\text { Tab. 2: } & \text { S. } 33\end{array}$

Tab. 3: $\quad$ S. 34

Tab. 4: $\quad$ S. 34

Tab. 5: $\quad$ S. 35

Tab. 6: S. 36

$\begin{array}{ll}\text { Tab. 7: } & \text { S. } 37\end{array}$

$\begin{array}{ll}\text { Tab. 8: } & \text { S. } 39\end{array}$

Tab. 9: $\quad$ S. 40

Tab. 10: $\quad$ S. 42

Tab. 11: $\quad$ S. 42

Tab. 12: $\quad$ S. 43

Tab. 13: $\quad$ S. 44

Tab. 14: $\quad$ S. 45

Tab. 15: $\quad$ S. 52

Tab. 16: S. 57

$\begin{array}{ll}\text { Tab. 17: } & \text { S. } 57\end{array}$ 


\section{Einleitung}

\subsection{Einführung in das Thema}

Schlafbezogene Atemregulationsstörungen haben in vergangenen Jahren zunehmend an Bedeutung gewonnen. Durch umfangreiche Forschungsarbeiten wurde deutlich, dass es sich hierbei um sehr komplexe Krankheitsbilder handelt, deren mögliche Komplikationen für den Allgemeinorganismus schwerwiegend sein können. Die internationale Klassifikation der Schlafstörungen (ICSD) fasst diese einzelnen Störungen systematisch zusammen und dient als Grundlage für die schlafmedizinische Diagnostik. Das obstruktive Schlafapnoesyndrom (OSAS), auf das im folgendem näher eingegangen werden soll, zählt zur Gruppe der intrinsischen Schlafstörungen.

Das obstruktive Schlafapnoesyndrom ist eine Krankheit, bei der trotz anhaltender Atemanstrengung der inspiratorischen Atemmuskulatur eine Unterbrechung des Atemstroms an Nase und Mund während des Schlafes auftritt (Schäfer 1996). Dadurch kommt es zum Auftreten von Apnoe- und Hypopnoephasen als Folge eines kompletten oder inkompletten Verschlusses der oberen Luftwege.

Die Symptome und Auswirkungen der OSAS sind vielfältig und haben Auswirkungen auf die Tagesbefindlichkeit, Leistungsfähigkeit, aber auch auf den psychosozialen und kardiovaskulären Bereich. Aus diesem Grund kommt dem Verständnis, der Diagnostik und der Therapie dieser Erkrankung eine besondere Bedeutung zu. Von Anfang an setzten Forscher und Kliniker eine große Zahl verschiedenster Untersuchungstechniken ein, um folgende Ziele zu erreichen: Unterschiede in der Anatomie der oberen Atemwege aufdecken, Ätiologie und Pathophysiologie der Erkrankung klären, sowie Patientenbetreuung und Behandlungserfolg verbessern. Mit statischen, bildgebenden Verfahren wie das laterale Fernröntgenseitenbild (Kephalometrie), Computertomographie (CT) und Magnetresonanztomographie (MRT) wurde vor allem versucht, anatomische Unterschiede zu detektieren.

Die vorliegende Arbeit beschäftigt sich vor allem mit der Bedeutung des Einsatzes von volumentomographischen Untersuchungen. Es soll herausgestellt werden, ob anatomische Strukturen und Veränderungen des Pharynx mit dem digitalen Volumentomographen (DVT) erkannt werden können und somit zur Diagnostik vom obstruktiven Schlafapnoesyndrom oder von Rhonchopathien dienen kann. 


\subsection{Schlaf, Schlafarchitektur und Schlafstörungen}

Schlaf ist definiert als ein schnell reversibler Zustand reduzierter Antwortbereitschaft auf Umgebungsreize und allgemein verminderter Interaktion mit der Umwelt. Schlaf existiert bei allen Säugetieren und füllt etwa $30 \%$ der Lebenszeit des Menschen aus (wovon im Mittel 25\% im Traumzustand verbracht werden). Eine wichtige Methode zur Analyse des menschlichen Schlafes ist die Elektroenzephalographie (EEG), deren Ergebnisse zur Einteilung verschiedener Schlafstadien führten (Silbernagel et al. 2005). Der Schlaf wird in 4 Schlafstadien eingeteilt.

Beim Übergang vom entspannten Wachzustand (mit geschlossenen Augen) in das Schlafstadium 1 verschwinden die sogenannten Alpha-Wellen im EEG. Die Klarheit des Bewusstseins wird zunehmend eingeschränkt. Viele Menschen erleben in diesem dösenden Übergangszustand zwischen Wachen und Schlafen optische, traumartige Eindrücke. Gleichzeitig beginnen sich die Augäpfel langsam hin- und herzubewegen. Bei manchen Schläfern zeigen sich beim Einschlafen auch feine Zuckungen der Augenlider. Es können aber auch heftige Zuckungen einzelner Gliedmaßen oder des ganzen Körpers auftreten, die wahrscheinlich durch eine Umstellung der motorischen Kontrollsysteme beim Einschlafen bedingt sind. Das Schlafstadium 1 ist noch ein instabiler Zustand, der leicht durch kurze Wachepisoden unterbrochen werden kann.

Der Beginn des nachfolgenden Schlafstadiums 2 ist daher als der eigentliche Zeitpunkt für den Schlafbeginn anzusehen, zumal hier zum ersten Mal Schlafspindeln und K-Komplexe im EEG auftreten. Die Zeitdauer zwischen Zubettgehen und dem Schlafstadium 2, also die Schlaflatenz, beträgt bei gesunden Erwachsenen etwa 10 bis 15 Minuten.

Normalerweise vertieft sich der Schlaf sukzessive aus den ersten beiden Schlafstadien in die Tiefschlafstadien 3 und 4. Die Weckschwelle für Reize erhöht sich entsprechend und erreicht ihren höchsten Wert nach etwa einer Stunde. Anschließend nimmt die Weckschwelle wieder ab. Schließlich geht der Tiefschlaf in den ersten REM (rapid eye movements)-Schlaf über, mit dem der komplette erste Schlafzyklus abgeschlossen wird. Der REM-Schlaf wird auch als paradoxer Schlaf bezeichnet, weil das EEG sich kaum vom Wachzustand unterscheidet, die Person aber regungslos mit geschlossenen Augen liegen bleibt. Es treten dabei sekundenlange Gruppen von 1-4 Hz schnellen Augenbewegungen auf. In dieser Zeit wird häufig aktivhandelnd und emotional geträumt, während in den übrigen Schlafphasen eher abstraktgedanklich geträumt wird (Schmidt et al. 2005). Die Schlafstadien 1 bis 4 werden als 
synchronisierter, langwelliger (,slow wave“) oder auch orthodoxer Schlaf bezeichnet (Silbernagel et al. 2005).

Im typischen Schlafverlauf einer Nacht werden die Perioden von synchronisiertem (Non REM (non-rapid eye movements)) und paradoxem (REM) Schlaf in regelmäßigen Zyklen etwa 5-7mal durchlaufen. Mit fortschreitender Schlafdauer werden die REM-Schlafphasen länger (maximale Dauer 30-50 Minuten), und die späteren Tiefschlafstadien des synchronisierten Schlafens (Stadien 3 und 4) werden nicht mehr erreicht. Spontanes, ungestörtes Aufwachen erfolgt meistens aus einer REM-Phase (obwohl die Schwelle für das Aufwecken durch äußere Reize während dieser Stadien hoch ist) und ist dann häufig mit der Erinnerung an ein Traumereignis verbunden (Silbernagel et al. 2005).

Die relativen Anteile von Wachen und Schlafen ebenso wie die Anteile von REM- und NonREM-Schlaf an der Gesamtschlafzeit machen eine charakteristische Altersentwicklung durch. Insgesamt sinkt im Laufe des Lebens nicht nur die Gesamtschlafzeit ab, sondern es wird auch der relative Anteil des Tiefschlafs erheblich kürzer (Schmidt et al. 2005).

Schlaf beeinflusst alle physiologischen Systeme wie das Herz-Kreislauf-System, die endokrine Funktion, die Thermoregulation, aber auch das neurologische System nachhaltig (Peter et al. 1995).

Schlafstörungen werden nach der ICSD (international classification of sleep disorders) der ASDA (American Sleep Disorders Association 1995) in 4 Gruppen unterteilt:

1. Dyssomnien:

a) intrinsische Schlafstörungen

b) extrinsische Schlafstörungen

c) Störungen des zirkadianen Schlafrhythmus

2. Parasomnien:

arousale Störungen, Störungen des Schlaf-Wachüberganges,

REM assoziierte Störungen, andere Störungen

3. Schlafstörungen bei körperlichen / psychischen / neurologischen Erkrankungen

4. andere Schlafstörungen.

Das obstruktive Schlafapnoesyndrom, auf das in der vorliegenden Arbeit näher eingegangen werden soll, zählt zu den intrinsischen Schlafstörungen und damit zu den Dyssomnien. 


\subsection{Anatomie des orofazialen Systems}

Das orofaziale System besteht aus dem Pharynx (Nasopharynx, Mesopharynx und Hypopharynx), sowie der Mundhöhle mit ihren Begrenzungen (Lippen, Zähnen, Gaumen, Kiefer und Zunge), der Nasenhöhle, der Trachea und dem Kehlkopf.

Die Trachea wird aus spangenförmigen Knorpelringen gebildet, die am Ende durch eine transversal verlaufende Muskulatur verspannt werden. Diese Bauweise ermöglicht es, einem sehr hohen negativen transmuralen Druck zu widerstehen, ohne zu kollabieren.

Der Trachea schließt sich der Larynx an, welcher neben der Nase eine der anatomischen Strukturen ist, die dem Luftstrom den höchsten Widerstand entgegensetzen. Dieser resultiert aus der Enge der Stimmritze, die von den Stimmbändern begrenzt wird.

Über dem Larynx beginnt mit dem Hypopharynx der Anteil der oberen Atemwege, der auf Grund seiner ganz unterschiedlichen Funktionen wiederum ein anderes Bauprinzip aufweisen muss. Luft, flüssige und feste Nahrung müssen gleichermaßen den Pharynx passieren können. Mit Hilfe dieser „elastisch-kollabierbaren Röhre“ (Hochban et al. 1995) und mit einer sehr gut koordinierten neuromuskulären Aktion ist es möglich, Nahrungsbestandteile in den Ösophagus zu befördern und nicht in die Trachea.

Der Hypopharynx wird anterior von der Epiglottis und dem Zungengrund gebildet. Die Epiglottis dient zum Verschluss des Kehlkopfeinganges. Sie ist nach anterior durch das Ligamentum hyoepiglotticum am Os hyoideum fixiert, wodurch der Hypopharynx offen gehalten wird. Das Os hyoideum ist ebenfalls durch eine Vielzahl von Ligamenten und von der infra- und suprahyoidalen Muskulatur in seiner Lage fixiert. Eine Kontraktion der suprahyoidalen Muskulatur führt $\mathrm{zu}$ einer Ventralverlagerung des Hyoides. Durch die Befestigung werden dann auch Zungengrund und Epiglottis nach anterior bewegt.

Posterior und lateral ist der Hypopharynx vom M. constrictor begrenzt, der sich weiter nach kranial erstreckt und dort den Oropharynx bildet. $\mathrm{Zu}$ den pharynxdilatierenden Muskeln gehören der M. genioglossus, der M. geniohyoideus, die Kaumuskulatur und der M. tensor veli palatini.

Anterior des Oropharynx befindet sich die Zunge. Sie wird aus den inneren und äußeren Zungenmuskeln gebildet.

Ist der Mund geschlossen, so füllt die Zunge die Mundhöhle nahezu völlig aus. Der weiche Gaumen lagert sich dem Zungenrücken an und bildet somit die anteriore Begrenzung des Nasopharynx. Dieser wird posterior durch die Wirbelsäule mit der paravertebralen Muskulatur gebildet sowie lateral vom Tonsillenbett, begrenzt vom vorderen und hinteren 
Gaumenbogen. Davor erstreckt sich die Nasenhöhle mit Septum und Nasenmuscheln. Auch dieser Teil stellt noch einmal eine Einengung für den Luftstrom dar.

Die kraniale Begrenzung der Mundhöhle bilden die knöchernen und weichen Strukturen des Gaumens. Nach lateral und ventral ist sie durch die Zähne mit dem Alveolarkamm und Wangen bzw. Lippen, nach kaudal durch die suprahyoidale Muskulatur begrenzt.

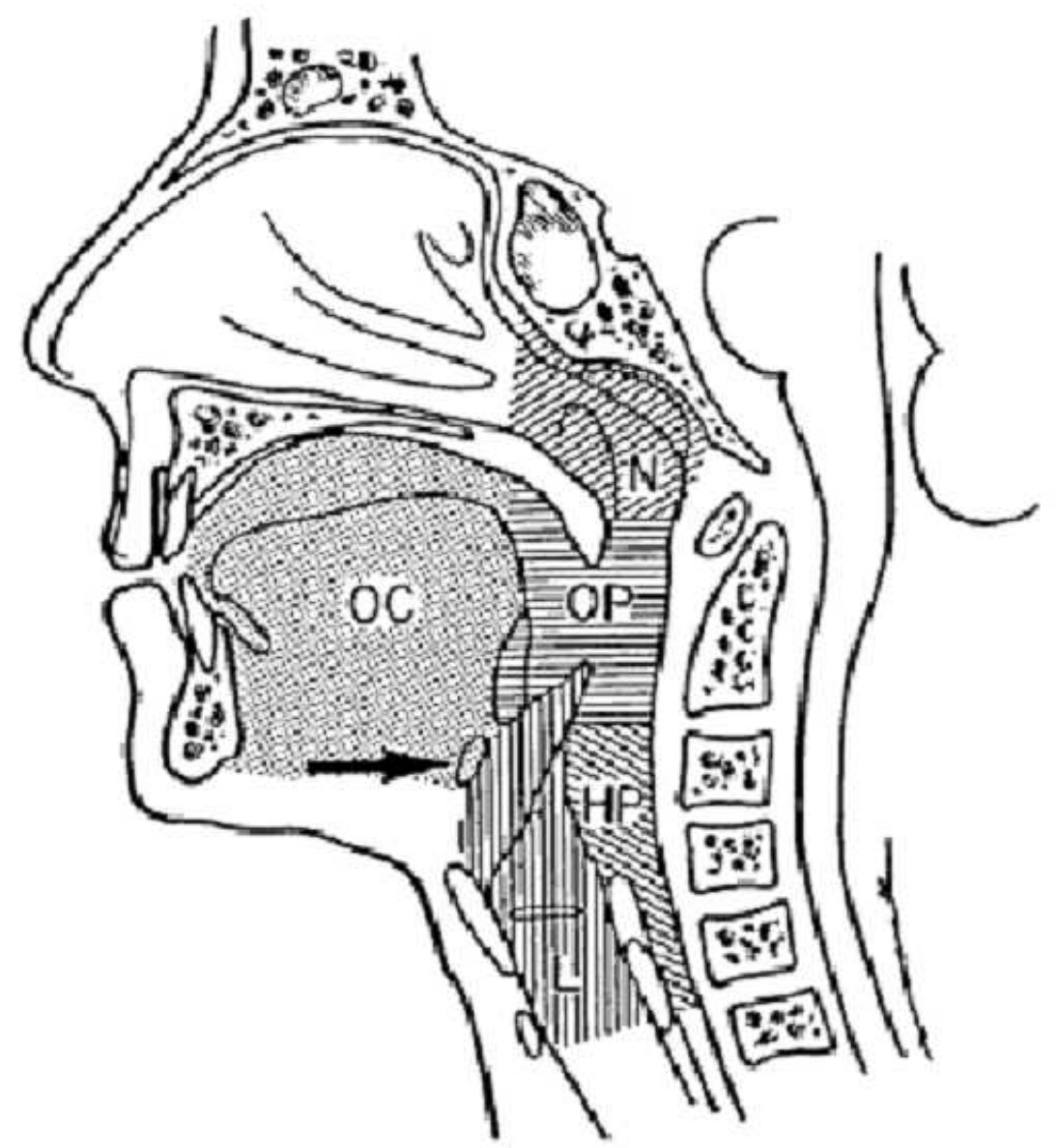

Abb. 1: Sagittales Schema des Pharynx

mit Einteilung entsprechend der anatomischen Lokalisation, N= Nasopharynx, OP= Oropharynx, HP= Hypopharynx, OC= Mundhöhle, L= Larynx, Pfeil= Os hyoideum. Entnommen aus Lomoschitz et al. (2000) Seite 603. 


\subsection{Das Schlafapnoe-Syndrom}

\subsubsection{Definition}

Charakteristisch für diese Art von intrinsischer Dyssomnie ist das rezidivierende Auftreten von Apnoe- und Hypopnoephasen, bedingt durch kompletten oder inkompletten Verschluss der oberen Luftwege im Bereich von Oro-, Naso- oder Hypopharynx (Guilleminault et al. 1976).

Die „klassische“ Definition des Schlafapnoesyndroms geht auf Guilleminault et al. (1978) zurück, wonach in einer siebenstündigen Nachtableitung mindestens 30 Apnoephasen von mehr als 10 Sekunden Phasendauer, sowohl im REM- als auch im Non-REM-Schlaf, auftreten müssen, um von einem Schlafapnoesyndrom sprechen zu können.

Hieraus entwickelt sich dann ein Apnoe-Hypopnoe-Index (AHI) von fünf Apnoen pro Stunde Schlafzeit $(\mathrm{AHI}=5)$ als Grenzwert, unterhalb dessen man noch von einem Normalbefund spricht (Guilleminault et al. 1993, Guilleminault et al. 1978). Nach Berry et al. (1984) und Gould et al. (1988) bewährt sich in der klinischen Praxis ein AHI-Grenzwert von 15 pro Stunde Schlaf. Andere Autoren legen die Grenze für OSAS bei mehr als 10 Apnoen pro Stunde fest (Schäfer und Lenders 1990). In der vorhandenen Literatur liegt der AHIGrenzwert zur Definition von OSAS-Patientengruppen zwischen einem AHI > 5 (Johal et al. (2007) und einem AHI > 25 (Fairburn et al. 2007) pro Stunde.

Unter einer Apnoephase versteht man das vollständige Sistieren des Atemflusses für mindestens 10 Sekunden. Neben den Apnoen werden bei den OSAS-Patienten auch oft sogenannte „Hypopnoen“ wahrgenommen. Eine Hypopnoe ist definiert als eine gleichfalls mindestens 10 Sekunden dauernde Abnahme der Ventilation um 50\%, verbunden mit einem Abfall der arteriellen Sauerstoffsättigung um mehr als 4\% (Schäfer und Lenders 1990, Haponik et al. 1983). Hierbei kommt der Atemfluss nicht völlig zum Erliegen, ist jedoch stark vermindert. Eine exakte Definition der Grenze zwischen noch Normalem und Pathologischem ist hier noch schwieriger, jedoch ist anerkannt, dass die pathophysiologische Wirkung nahezu gleich derer der Apnoe ist (Gould et al. 1988).

Schon Gastaut et al. (1966) nahmen eine Einteilung in zentrale, obstruktive und gemischte Apnoen vor. Die zentrale Form ist phänomenologisch durch das Erliegen von Nasen- und Mundatemflow sowie der Atembewegung im Thorax- und Abdominalbereich charakterisiert. Der zentrale Atemantrieb bleibt hierbei aus, bedingt durch die neurale Dysfunktion des 
Atemregulationszentrums und es kommt somit $\mathrm{zu}$ einem völligen Sistieren aller an der Atmung beteiligter Muskelgruppen (Strollo und Rogers 1996).

Von obstruktiver Apnoe wird im Fall einer zentralen Aktivierung ausschließlich des Zwerchfells und der unteren Thoraxmuskulatur bei gleichzeitiger Okklusion des Oropharynx gesprochen (Herold 2000, Andersson und Brättström 1991). Hierdurch kommt es zu erheblichen intrathorakalen Druckschwankungen (Gastaut et al. 1966).

Das gemischte Apnoemuster stellt eine Kombination aus zentraler und obstruktiver Form dar, wobei charakteristisch die zentrale Form der Auslöser ist, gefolgt von der obstruktiven Komponente (Strollo und Rogers 1996).

Bei einer sehr großen Zahl der erwachsenen Patienten mit einem Schlafapnoe-Syndrom nimmt die pharyngeale Obstruktion eine große, wenn nicht die Hauptrolle ein. Hierbei spricht man von einem ,,vorherrschend obstruktiven Schlafapnoesyndrom“. Quantitativ kann die Hauptzahl jedoch als phänomenologisch gemischt klassifiziert werden. Die rein zentrale Form kommt hingegen nur selten vor (Peter et al. 1991).

\subsubsection{Epidemiologie}

Zahlreiche epidemiologische Studien der vergangenen Jahre zeigen, dass die Schlafapnoe häufiger vorkommt als zuvor angenommen. Diese Untersuchungen lassen eine hohe Prävalenz dieser Erkrankung erkennen. In der Bundesrepublik Deutschland wird sie von Peter et al. (1990) auf 1 - 3\% geschätzt. Ähnliche Werte werden auch von anderen Autoren für weitere Länder angegeben (Young et al. 2002, Bixler et al. 2001, Gislason et al. 1988, Lavie 1983).

Einige neuere Untersuchungen geben eine höhere Prävalenz von bis zu 19\% der Männer und 15\% der Frauen an (Plywaczewski et al. 2007, Duran et al. 2001).

Der Altersgipfel liegt bei den Männern zwischen dem 40. - 49. Lebensjahr und bei den Frauen zwischen dem 50. - 60. Lebensjahr. Insgesamt sind die Männer jedoch häufiger betroffen als die Frauen (Young et al. 1993, Block et al. 1989). Nach Partinen und Telakivi (1992) ist die Prävalenz beim männlichen Geschlecht zwischen 40 und 65 Jahren am höchsten und kann $8,5 \%$ oder mehr betragen. Studien haben gezeigt, dass nach dem 65 . Lebensjahr die Prävalenz abnimmt (Young et al. 2002, Lugaresi et al. 1980).

Das Geschlechterverhältnis Mann zu Frau wird von verschiedenen Autoren zwischen 4:1 und 10:1 angegeben (Plywaczewski et al. 2007, Partinen und Telakivi 1992, Lavie 1983, Sackner et al. 1975). 
Lindberg und Gislason (2000) geben eine Prävalenz von 0,5 - 2\% bei Frauen vor der Menopause an. Bei den Frauen wird jedoch ein deutlicher Anstieg der OSAS-Inzidenz nach der Menopause beschrieben, besonders bei Frauen ohne Hormontherapie, was auf einen protektiven Effekt hormoneller Faktoren schließen lässt. Trotzdem bleibt die Anzahl der betroffenen Frauen geringer als bei den Männern (Lamberg 2007, Young et al. 2003, Jordan und McEvoy 2003, Bixler et al. 1998).

\subsubsection{Symptome und Folgen}

Das klinische Bild von OSAS beinhaltet 4 Hauptsymptome: starkes Schnarchen, erhöhte Tagesschläfrigkeit, häufige nächtliche Erweckungen mit Nykturie und morgendliche Asthenie (Kraftlosigkeit) mit oder ohne Kopfschmerzen (Baguet et al. 2009). Die Leitsymptome sind meist ein fremdanamnestisch berichtetes lautes und unregelmäßiges Schnarchen mit Atempausen (Noda et al. 1998 a).

Wissenschaftliche Untersuchungen der letzten Jahre haben kausale Verbindungen zwischen OSAS und arterieller Hypertonie (Baguet et al. 2009, Peppard et al. 2000), Insulinresistenz (Onat et al. 2007, Punjabi und Polotsky 2005, Coughlin et al. 2004, Punjabi et al. 2004, Ip et al. 2002) beschrieben. Es wird angenommen das OSAS aufgrund der kardiovaskulären Morbidität (Wolk et al. 2003, Shahar et al. 2001) mit einer erhöhten Mortalität einhergeht (Hader et al. 2006, Yaggi et al. 2005). Das Mortalitätsrisiko ist durch kardiorespiratorische Veränderungen, vor allem bei Patienten mit einem Apnoe-Index $>20$ pro Stunde und einer Sauerstoffentsättigung < $72 \%$ deutlich erhöht (Guilleminault et al. 1996). Herold (2000) gibt für unbehandelte Patienten dieser Risikogruppe eine 8-Jahremortalitätsrate von $40 \%$ an. Arzt et al. (2005) und Wessendorf et al. (2000) haben ein erhöhtes Auftreten von zerebrovaskulären Krankheiten bei OSAS-Patienten festgestellt. Außerdem treten Persönlichkeitsveränderungen, sexuelle Funktionsstörungen und verminderte Konzentration auf (Schäfer 1996).

Die sozioökonomische Bedeutung dieser Schlafstörung wird durch die mit ihr assoziierte Schläfrigkeit und dadurch verursachte Unfälle unterstrichen (Kingshott et al. 2004, Pack und Pien 2004). Nach Findley et al. (1988) haben Schlafapnoe-Patienten gegenüber Gesunden eine 7-fach höhere Unfallrate. Unbehandelte Schlafapnoe-Syndrome verursachen in den USA schätzungsweise bis zu 3,4 Milliarden US-Dollar zusätzliche medizinische Kosten (Kapur et al. 1999). Die Gesamtkosten für schlafbezogene Unfälle in den USA werden von Leger (1994) auf 43 bis 56 Milliarden US-Dollar geschätzt. Eine besondere Stellung nehmen 
Straßenverkehrsunfälle ein. Sie sind die häufigste Unfallursache mit Todesfolge. Neben Alkohol am Steuer stellen u.a. Müdigkeit bzw. Einschlafen während des Fahrens die Hauptursache dar. So sind $24 \%$ aller tödlichen Unfälle auf bayrischen Autobahnen im Jahre 1991 auf Einschlafen am Steuer zurückzuführen (Langwieder et al. 1994). Nach Aldrich (1989) beträgt die Inzidenz von schlafbezogenen Autounfällen wegen exzessiver Tagesschläfrigkeit 3-7\%. Demnach ist Einschlafen am Steuer ein wichtiger Faktor für das erhöhte Unfallrisiko von Schlafapnoe-Patienten.

Die vielfältigen Symptome und Spätfolgen der Schlafapnoe beruhen auf drei grundlegenden Folgen der Apnoe: der Hypoxämie, den starken intrathorakalen Unterdrücken und den auftretenden Weckreaktionen, den so genannten Arousals am Ende der Atempause.

Durch die bis zu 120 Sekunden langen Apnoephasen kann die arterielle Sauerstoffsättigung bis unter $30 \%$ der Normwerte absinken. Eine hierdurch mögliche hypoxische Schädigung von Herzmuskel- und Gehirnzellen durch diese ständig stattfindende Sauerstoffentsättigung stellt einen Pathomechanismus dar, der wenigstens zum Teil für die zahlreichen internistischen Folgeerkrankungen verantwortlich ist (Noda et al. 1998 b).

Die starken intrathorakalen Unterdrücke können ein Vielfaches des Normalwertes einnehmen. So werden von bis $\mathrm{zu}-150 \mathrm{~mm} \mathrm{H}_{2} \mathrm{O}$ erreicht, was dem Fünfzehnfachen des Normalwertes entspricht (Cirignotta und Lugaresi 1980). Die Folge ist eine Bradykardie mit pulmonaler Hypertonie während der Apnoe, die durch den vermehrten Blutzustrom zum Herzen und vagale Reflexe zustande kommt.

Auch die ständig auftretenden Arousals werden als Ursache für die arterielle Hypertonie bei Schlafapnoepatienten diskutiert (Shepard 1992).

Die genannten drei Faktoren müssen im Zusammenhang betrachtet werden, da sie sich in ihrem Pathomechanismus beeinflussen und zum Teil verstärken. So bewirken sie zusammen arterielle Blutdruckspitzen und kardiale Arrhythmien (Ingbar und Gee 1985).

Durch die Abnahme der Blutsauerstoffkonzentration kommt es zu einer Weckreaktion, ein so genanntes Arousal, das zur einer Tonisierung der Schlund- und Halsmuskulatur führt und die Atemwege wieder freigibt (Gleeson et al. 1990). Nach Soler und Tamm (1995) ist das Arousal die letzte respiratorische Reaktion auf die Apnoe, ohne die es zu schweren Hypoxämien oder sogar zum Tod kommen kann. Nach einigen tiefen Atemzügen kommt es zu einer Normalisierung der Blutgase. 
Eine weitere Folge des Arousals ist die Schlaffragmentierung, wodurch die normalen Leicht-, Tief- und REM-Schlafphasen nicht in physiologischer Form durchlaufen werden.

Nach einer ASDA-Definition (American Sleep Disorders Association 1992) ist ein Arousal jede abrupte Änderung der EEG-Frequenz, die Theta- $(4-7 \mathrm{~Hz})$, Alpha- (8-13 Hz) und /oder Frequenzen über $16 \mathrm{~Hz}$ umfasst, aber keine Spindeln einschließt. Des Weiteren muss der Patient vor dem Arousal 10 Sekunden schlafen unabhängig vom vorausgehenden Schlafstadium. Weiterhin ist es nötig, dass zwischen zwei Arousals 10 Sekunden Schlaf liegen und die Änderung des EEG mindestens 3 Sekunden lang sein muss. In Non-REMSchlafphasen kann ein Arousal ohne EMG-Aktivierung auftreten, in REM-Schlafphasen ist das Vorhandensein einer EMG-Aktivierung notwendig.

Allerdings bedingen EMG-Änderungen alleine, Artefakte, K- Komplexe, Deltawellen (0,5- 3 $\mathrm{Hz}$ ) oder die Aktivität von Alphawellen von unter 3 Sekunden kein Arousal.

\subsubsection{Lokale pathophysiologische Zusammenhänge der Atemwege}

$\mathrm{Zu}$ den allgemeinen Risikofaktoren gehört zunehmendes Alter. Weiterhin spielen das männliche Geschlecht, Alkoholkonsum sowie die Einnahme von Sedativa und Hypnotika eine wichtige Rolle (Schäfer 1996). In Studien wurde ein Zusammenhang zwischen Übergewicht bzw. erhöhtem Body-Mass-Index (BMI) und OSAS festgestellt (Pracharktam et al. 1996, Schäfer 1996, Partinen et al. 1988, Riley et al. 1983).

Angesichts der Ausführungen zur Anatomie der oberen Atemwege ergeben sich im Wesentlichen vier mögliche pathophysiologische Faktoren, die zu einer Einengung bzw. zu einem Kollaps des Luftraumes beitragen können:

1. Pharyngealer Unterdruck und Kollapsibilität der umliegenden Strukturen,

2. Einengung bzw. Verlegung des Lumens,

3. Skelettale Veränderungen,

4. Aktivität bzw. fehlender Tonus der oberen Atemwegsmuskulatur.

Jeder dieser vier Faktoren für sich allein mag für den pharyngealen Kollaps unzureichend sein. Ein enger Pharynx muss nicht zwangsläufig leichter kollabieren. Aber in Kombination können strukturelle und funktionelle Faktoren zusammen zur Obstruktion führen (Hoffstein und Slutsky 1987). 
Der intrapharyngeale Druck bei der Inspiration wird vom Luftfluss und vom Widerstand der Nasenöffnung bzw. einer weiteren funktionell bedeutsamen Engstelle bestimmt. Abhängig vom Widerstand entsteht gemäß dem Bernoullischen Gesetz ein Unterdruck gegenüber der Umgebung, der auf alle kollabierbaren Anteile des Pharynx wirkt (Sukerman und Healy 1979). Der Pharynx ist in diesem Zusammenhang als potenziell kollapsgefährdeter Weichteilschlauch anzusehen, dessen freies Lumen innerhalb gewisser Grenzen durch transmurale Druckgradienten bestimmt wird. Der inspiratorisch leicht negative Druck im Pharynx muss durch den Tonus und die phasische, inspiratorische Aktivierung der den Pharynx dilatierenden Muskulatur aufgefangen werden (Soler und Tamm 1995). Störungen, der sich in der Pharynxwand befindenden Mechanorezeptoren oder ihrer Reflexbahnen erleichtern die Kollapsneigung (Ryan und Bradley 2005).

Die Auswirkung des Unterdrucks wird wesentlich beeinflusst durch die Elastizität des Pharynx, wobei die klinischen Untersuchungen am wachen Patienten offensichtlich keine Rückschlüsse auf die Verhältnisse im Schlaf zulassen (Mayer-Brix et al. 1988). Die Kollapsstelle ist nicht immer gleich und ist unter anderem auch von den Schlafstadien abhängig (Shepard und Thawley 1990).

Eine Einengung oder Verlegung des Lumens im Bereich des oberen Luftweges ist möglich. Hierbei können mechanische Faktoren wie Hypertrophie der Tonsillen und Zunge (Ryan und Bradley 2005), Zysten oder Tumore eine Rolle spielen, aber auch die Schlaflage.

So wirken sich Lageveränderungen auf den Atemwiderstand und das pharyngeale Lumen aus und es konnte gezeigt werden, dass das Lumen des Pharynx in Rückenlage am kleinsten ist und dass es sich in Seitenlage und im Sitzen vergrößert (Miki et al. 1988, Brown et al. 1987). Eine Einengung ist auch durch die Einlagerung von Fett im Pharynxbereich möglich, wie es bei Schlafapnoikern im Vergleich zu Gesunden computertomographisch und mit Hilfe der Magnetresonanztomographie sowie auch histologisch dargestellt werden konnte (Edström et al. 1992, Polo et al. 1991, Horner et al. 1989).

Skelettale Veränderungen sind einer der Hauptfaktoren der Verengung des Luftraumes. Die anatomische Basis für pharyngeale Obstruktionen ist das Skelett des Gesichtsschädels, an dem die Pharynxteile fixiert sind (Hollowell und Suratt 1991).

Patienten mit obstruktiver Schlafapnoe weisen anatomische Abnormalitäten im kraniomandibularen und oberen Luftwegsbereich auf (Partinen et al. 1988). Studien belegen, dass diese Abnormalitäten in der skelettalen Morphologie und im angrenzenden 
Weichgewebe (Johal und Conaghan 2004, Battagel und L'Estrange 1996) liegen. Die häufigsten Gemeinsamkeiten der OSAS-Patienten sind Hypoplasie und/oder Dorsalverlagerungen des Ober- und Unterkiefers (Ferguson et al. 1995). Einen öfter auftretenden retrognathen Gesichtstyp bei OSAS-Patienten bestätigen auch andere Autoren (Sakakibara et al. 1999, Hochban et al. 1994, Lowe et al. 1986), sowie eine reduzierte Unterkieferlänge (Johal et al. 2007, Battagel und L'Estrange 1996). Zudem wurde ein vergrößerter kranio-zervikaler Winkel (Solow et al. 1996), ein verkleinerter Abstand zwischen unteren Inzisivi und posteriorer Pharynxwand (Johal et al. 2007, Battagel und L'Estrange 1996), eine längere und dickere Uvula bzw. Velum (Johal et al. 2007, Tangugsorn et al. 1995, Hochban et al. 1994), eine vergrößerte Zunge (Johal et al. 2007, Battagel et al. 2000, Tangugsorn et al. 1995), eine deutlich tiefere und dorsal verlagerte Vallecula (Hochban et al. 1994), ein nach inferior verlagertes Zungenbein (Oosterkamp et al. 2007, Johal et al. 2007, Partinen et al. 1988, Riley et al. 1983) bzw. eine vergrößerte Distanz zwischen Mandibularebene und Hyoid (Partinen et al. 1988) beobachtet.

Mittelgesichtshypoplasien können sich ebenfalls negativ auf das Lumen der oberen Atemwege auswirken. Dies ist zum Beispiel der Fall beim Down-Syndrom, wo noch zusätzlich eine muskuläre Hypotonie hinzukommt (Hultcrantz und Svanholm 1991).

Als Folge dieser anatomischen Abnormalitäten liegt ein verengter pharyngealer Luftweg auf allen Ebenen zwischen Nasallinie und Mandibularplanum in der Sagittalen (Johal et al. 2007, Tangugsorn et al. 1995, Battagel und L'Estrange 1996, Hochban et al. 1994, Riley et al. 1983), sowie auch in der Transversalen (Shen 1993) vor. Diese anatomische Verengung des Luftweges wird durch den erhöhten Muskeltonus der Pharynx- und der Zungenmuskulatur im Wachzustand kompensiert (Fogel et al. 2004).

Die Aktivität der oberen Atemwege ist ein weiterer Faktor, der zu einem Kollaps des Lumens des Luftweges führen kann. Die intrinsische Pharynxmuskulatur, die Gaumenmuskulatur, die Zungenbein- sowie die Kaumuskulatur werden vom Zentralnervensystem aktiviert (Harston und Sauerland 1981). Über Reflexbahnen aktiviert, bewirkt die Muskulatur der oberen Atemwege nicht nur ein Offenhalten, sondern auch die aktive Öffnung des Pharynx, wenn er kollabiert ist (Grassino und Begin 1990). Lokale, über Mechanorezeptoren gesteuerte Reflexbahnen beeinflussen die Aktivität der pharynxdilatierenden Muskulatur. Negative Drücke bewirken eine Aktivitätssteigerung dieser Muskeln (Horner et al. 1991). 
Kollapsbegünstigend muss die auch physiologisch vorkommende funktionelle Tonusabnahme der Muskulatur im Schlaf berücksichtigt werden, die am stärksten im REM-Schlaf und geringfügiger im Tiefschlaf ausgeprägt ist. Diese verminderte Muskeltonisierung wurde von Tangel et al. (1991) auch für die Gaumenmuskulatur nachgewiesen. Von SchlafapnoePatienten kann sie nur unzureichend kompensiert werden. Letztere ist die wohl wichtigste Komponente für den Kollaps der oberen Atemwege und letztlich die Erklärung für die nur während des Schlafs auftretenden Okklusionen (Issa und Sullivan 1984). Die Abnahme des Muskeltonus wird verstärkt durch Alkohol und Sedativa (Remmers et al. 1978). Die durch die Obstruktion auftretende Abnahme der Blutsauerstoffkonzentration bewirkt eine Weckreaktion, ein so genanntes Arousal, die zu einer Tonisierung der Schlund- und Halsmuskulatur führt und die Atemwege wieder freigibt (Gleeson et al. 1990).

\subsubsection{Diagnostik und Therapie}

Bei der Diagnostik des OSAS hat sich ein gestuftes Vorgehen etabliert, dessen Ziel es ist, raschen Aufschluss über die Dringlichkeit einer polysomnographischen Untersuchung zu erhalten. Neben der Anamnese, die die wichtigsten Symptome der Schlafapnoe und mögliche Begleiterkrankungen enthalten sollte, ist es sinnvoll, auch standardisierte Fragebögen wie z.B. die Epworth Sleepiness Scale (Johns 1993) oder den Siegrist-Fragebogen (Siegrist et al. 1987) zu verwenden. In der Tagesdiagnostik müssen zahlreiche klinische Untersuchungen vorgenommen werden. In der Nachtdiagnostik erfolgt zunächst ein „Screening“ mit einem sog. Nicht-Labor-Monitoring-System, welches zur Erhärtung der Verdachtsdiagnose, zum Teil der Differentialdiagnostik und der gezielten Indikationsstellung zur Polysomnographie (PSG) dient. Als letzter Schritt zur Verifizierung eines OSAS ist die PSG unumgänglich. Sie dient der Diagnosesicherung, der Differentialdiagnostik und der Einschätzung des Schweregrades und stellt den Goldstandard der schlafmedizinischen Diagnostik dar (Johal und Conaghan 2004).

Die Polysomnographie ist eine Untersuchung und Messung bestimmter biologischer Parameter im Schlaf. Sie eignet sich zur Differentialdiagnose von Schlafstörungen und Schlafapnoe-Syndromen. Eine Polysomnographie wird in speziell eingerichteten Schlaflaboren unter stationären oder ambulanten Bedingungen durchgeführt.

Vor der Durchführung der Messungen im Schlaflabor wird der Patient in der Regel für einen Tag beobachtet, um seine Aktivitäten und Gewohnheiten zu erkennen. Normalerweise werden dann zwei Nächte im Labor verbracht. 
Bei einer stationären Polysomnographie werden nach Bedarf folgende Überwachungen standardmäßig durchgeführt (http://de.wikipedia.org/wiki/Polysomnographie (24.1.11)):

- Elektroenzephalographie (EEG)

- Elektrokardiogramm (EKG)

- Sauerstoffgehalt des Blutes (Pulsoxymetrie)

- Körpertemperatur

- Atemfluss (Mund und Nase)

- Atmungsbewegung

- Elektromyographie (EMG)

- Beinbewegung

- Augenbewegung (Elektrookulographie (EOG))

- Körperlage.

Zusätzlich können folgende Maßnahmen bzw. Messungen während des Schlafes vorgenommen werden:

- Tonaufzeichnung

- Videoaufnahmen

- Blutdruck

- Druckunterschiede im Brustkorb

- Erektionen

- Maskendruck für Atemmasken.

Durch die Polysomnographie können die Dauer und Häufigkeit von Apnoen und Hypopnoen ermittelt werden, ebenso das Ausmaß der Blutsauerstoffentsättigung, der Schlafstadien und deren Beziehung sowie die Differenzierung der Apnoegenese (Andreas et al. 1993).

Tabelle 1 zeigt das Stufendiagnostikschema.

\section{Anamnese}

- Fragebogen

- Ggf. Tagebuch zum Schlaf-Wach-Verhalten

\section{Tagesdiagnostik}

- Neuropsychologische und -physiologische Untersuchungen

- Kardiovaskulärer und -pulmonaler Status

- Humoraler Status (z.B. TSH-basal)

- Neurologische Untersuchungen

- Hals-Nasen-Ohren- und Mund-Kiefer-Gesichts-Status

Nachtdiagnostik

- Screening

- Polysomnographie

Tab. 1: Systematik der Diagnostik beim OSAS (Rasche et al. 1999) 
Den derzeitigen therapeutischen Standard, definiert von der Deutschen Gesellschaft für Pneumologie, bildet eine Drei-Stufen-Therapie, welche nach dem Schweregrad des Schlafapnoesyndroms ausgerichtet ist (Fischer 1991, Peter et al. 1991).

Die erste Stufe beinhaltet allgemeine und präventive Maßnahmen. So wird im Besonderen auf die Schlafhygiene, Gewichtsreduktion, Alkohohl- und Sedativakarenz und das Vermeiden ungünstiger Lagen im Schlaf geachtet (Lojander et al. 1998, Soler und Tamm 1995).

In der zweiten Stufe ist eine geringe medikamentöse Therapie möglich. Es sollten auch bereits vorliegende Begleiterkrankungen wie die arterielle Hypertonie behandelt werden. Nach Peter et al. (1992) lassen sich mit den ersten beiden Therapiestufen etwa $60-70 \%$ der Schlafapnoe-Patienten behandeln. Andere Autoren geben an, von medikamentösen Behandlungen sei im allgemeinen kein Langzeiteffekt $\mathrm{zu}$ erwarten. Eine deutliche Gewichtsreduktion sei oft wirksam und bei Adipositas immer anzustreben, aber nur selten zu erreichen (Soler und Tamm 1995).

In der dritten Stufe werden konservative oder chirurgische Therapien angewandt. Eine Möglichkeit der konservativen Behandlung ist die nasale, kontinuierliche Atemüberdruckbeatmung (nasal continuous positive airway pressure $=n$-CPAP). Diese Therapie wurde von Sullivan et al. (1981) entwickelt und war die erste nicht invasive Methode, die den Kollaps der oropharyngealen Muskulatur im Schlaf durch die sogenannte „,pneumatische Schienung“ verhinderte. Mit dem Druck zwischen 50-150 mm H2O, appliziert über eine Silikonmaske, kann der Kollaps des Pharynx meist verhindert werden und der Schlaf normalisiert sich. Sie wird als Methode der Wahl zur Behandlung schwerer obstruktiver Schalfapnoe weltweit anerkannt und als sehr effektiv eingeschätzt (Podszus 1993, Peter et al. 1991) und hat sich in den letzten Jahren etabliert (Sullivan et al. 1984, Sullivan et al. 1981).

Wilcox et al. (1993) wiesen eine signifikante Senkung des systolischen und diastolischen Blutdrucks nach. Mortimore et al. (1996) konnten nachweisen, dass sich nach Langzeittherapie die oberen Luftwege vergrößerten. Die individuelle Therapieeinstellung und die regelmäßigen Kontrollen sollten in einem Schlaflabor unter polysomnographischer Kontrolle erfolgen. Die Nebenwirkungen sind in der Regel nur von lokaler Natur, gut therapierbar und führen nur selten zu einem Therapieabbruch. Beschrieben werden allgemeine Nebenwirkungen wie Beeinträchtigung durch Geräusche der Geräte, Maskenprobleme, Eintrocknung der Nasenschleimhäute und Reizung der Augen. Ernsthafte Komplikationen sind selten. Als absolute Kontraindikation sind unkooperative Patienten anzusehen. Als relative Kontraindikationen gelten lokale Faktoren, die eine Therapie erschweren oder 
unmöglich machen, wie z.B. eine laxe Epiglottis oder Tumoren des Nasen-Rachen-Raums (Rasche et al. 1999).

Eine weitere apparative Behandlung von OSAS ist die Esmarch-Schiene oder ähnliche Geräte zur Protrusion des Unterkiefers und der Zunge (Hochban et al. 1995, Schmidt-Nowara et al. 1991, Lowe et al. 1990), wodurch es zu einer Erweiterung des Pharynxlumens kommt. Eine Heilung durch diese Protrusionsbehelfe ist nicht möglich, sodass die Behandlung lebenslang nachts angewandt werden muss (Hochban et al. 1994), ebenso wie die n-CPAP-Therapie.

Die chirurgische Behandlung des OSAS zielt auf die Beeinflussung der Ursachen des Pharynxkollapses ab. So können bei im Vordergrund stehender Widerstandserhöhung im Nasenbereich operative Maßnahmen die Atemstörung und Symptomatik gelegentlich bessern. Ein weiterer Therapieansatz stellt die chirurgische Korrektur von Weichteilveränderungen dar. Bei den seltenen Patienten mit offensichtlichen engen Verhältnissen im Mesopharynx kann eine Tonsillektomie und gegebenenfalls Uvulo-Palato-Pharyngo-Plastik (UPPP) die Neigung zur Luftwegsokklusion vermindern (Fujita et al. 1981). Der Behandlungserfolg bei diesen operativen Maßnahmen kann aber bei weitem nicht garantiert werden (Rodenstein 1992, Walker et al. 1989, Harmon et al. 1989).

Als weitere chirurgische Behandlung ist eine Ventralverlagerung des Ober- und Unterkiefers beschrieben worden (Fairburn et al. 2007). Durch die Vorverlagerung des Unterkiefers und der sich daran befindlichen Weichteile wie Mundboden und Zungenmuskulatur ist es möglich, den Pharynxquerschnitt zu vergrößern.

Als ein effektives Verfahren hat sich die in Göttingen entwickelte funktionelle Palatoraphie (Gaumenraffung) in Verbindung mit einer modifizierten Kinnosteotomie herausgestellt. Mit diesem Verfahren kann eine pathologische Verlängerung des Weichgaumens operativ erfolgreich beseitigt werden (Wiltfang et al. 1996).

Im Rahmen der chirurgischen Behandlung der obstruktiven Schlafapnoe hat sich die Radiofrequenzchirurgie als wirksam erwiesen. Hierbei wird hochfrequenter Wechselstrom zur Koagulation oder zum Schneiden von Gewebe angewandt (Hochfrequenz-Chirurgie). Bei der submukösen Anwendung kommt es zur Hitzekoagulation, die später vernarbt, wodurch eine Straffung des Gewebes erreicht wird. Diese Technik wird an den Nasenmuscheln, am Weichgaumen, an den Tonsillen und am Zungengrund angewandt (Verse et al. 2009).

Ein neues Operationsverfahren, das Atemaussetzer während des Nachtschlafs verhindern soll, erprobt seit 2008 die Hals-Nasen-Ohren-Klinik am Mannheimer Universitätsklinikum. Dabei wird ein Implantat in die Zunge eingebracht, das verhindert, dass die Zunge bei entspanntem 
Muskelzustand während des Schlafs zurückfällt und damit die Atemwege blockiert (http://www.innovations-report.de/html/berichte/studien/zungenimplantat_gegen_naechtliche atemaussetzer_118043.html (24.1.2011)).

\subsection{Bildgebendes Verfahren: Digitaler Volumentomograph (DVT)}

Die digitale Volumentomographie wurde 1997 als neue Aufnahmetechnik für die Zahn-, Mund- und Kieferheilkunde entwickelt und bietet die gleichen Rekonstruktionsmöglichkeiten für die Hartgewebsdiagnostik wie in der Computertomographie, scheint aber mit einer geringeren Strahlenexposition verbunden zu sein (Möbes et al. 2000, Arai et al. 1999, Mozzo et al. 1998). Sie wurde zur Darstellung der knöchernen Strukturen am menschlichen Schädel entwickelt, wobei gleichzeitig die hohe Strahlenbelastung der CT vermieden werden sollte (Scarfe et al. 2006, Cohnen et al. 2002).

Bei der digitalen Volumentomographie wird im Gegensatz zur CT, das stationäre Objekt durch eine einmalige Rotation $\left(360^{\circ}\right)$ der Sender-Detektoreinheit mit einem kegelförmigen Strahlenbündel (Conebeam-Technik) erfasst (Goch 2005, Voßhans 2005) (Abb. 2). Bei der Aufnahme führt das Gerät bei jedem Grad eine Messung durch. Durch den gepulsten Röntgenstrahl liegt die tatsächliche Zeit der Strahlenexposition nur bei einem Bruchteil der Umlaufzeit, die restliche Zeit dient der Ausrichtung der Röntgenröhre.

Die Expositionszeit ist durch diese Röntgentechnik bei den Geräten wesentlich geringer, als bei Standard-CT-Aufnahmen, wie sie in der Klinik zur Anwendung kommen (Rother 2006).

Anschließend wird aus 360 einzelnen Projektionsradiographien ein dreidimensionaler Datensatz rekonstruiert. Durch die Archivierung lassen sich beliebig viele Primärrekonstruktionen aus einem Rohdatensatz erstellen.

Die digitale Volumentomographie eignet sich nicht zur Weichteildiagnostik, insbesondere wegen der Strahlenqualität und der Rauschanteile (Thiel und Hassfeld 2001), erfüllt allerdings alle Anforderungen an die Hartgewebsdiagnostik im Zahn-, Mund- und Kieferbereich (Möbes et al. 2000, Mozzo et al. 1998). Bisherige Hauptanwendungsgebiete für die DVT sind die implantologische Planung (Möbes et al. 2000, Möbes et al. 1999), die Darstellung des Nervkanals der Mandibula vor operativer Entfernung der Weisheitszähne (Heurich et al. 2002) und traumatologische Fragestellungen (Flinzberg et al. 2003, Fuhrmann et al. 2003). Für Fragestellungen im kieferorthopädischen Bereich haben Hatcher und Aboudara (2004) die Anwendungsmöglichkeiten der DVT beschrieben. Im Gegensatz zur Computertomographie werden beim DVT keine Hounsfield-Einheiten berechnet. 
Die Daten der Primärrekonstruktion werden im Dicom-Format ausgegeben und können so in Programme zur computerunterstützten Implantologie eingelesen werden.

Die zurzeit auf dem Markt befindlichen Modelle unterscheiden sich in der Patientenpositionierung (liegend oder aufrecht), in der Aufnahmedauer und Größe des Scanbereiches (Scherer et al. 2007). Die Größe des Scanbereiches der derzeitig angebotenen Geräte variiert zwischen $3 \times 4 \mathrm{~cm}$ (Morita 3 D Accutiomo) bis zu 20x20 cm (NewTom $3 \mathrm{G}-$ MF 12) (Dirsch 2008).

Das von der Firma Orangedental vertriebene DVT PaX-Reve3D erlaubt es dem Behandler, zwischen 4 Aufnahmegrößen $\mathrm{zu}$ entscheiden $(5 \times 5 \mathrm{~cm}, 8 \times 6 \mathrm{~cm}, 12 \times 8 \mathrm{~cm}, 15 \times 15 \mathrm{~cm})$ (http://www.orangedental.de/index.php/3d-digitales-roentgen/pax-reve3d (1.11.10)).

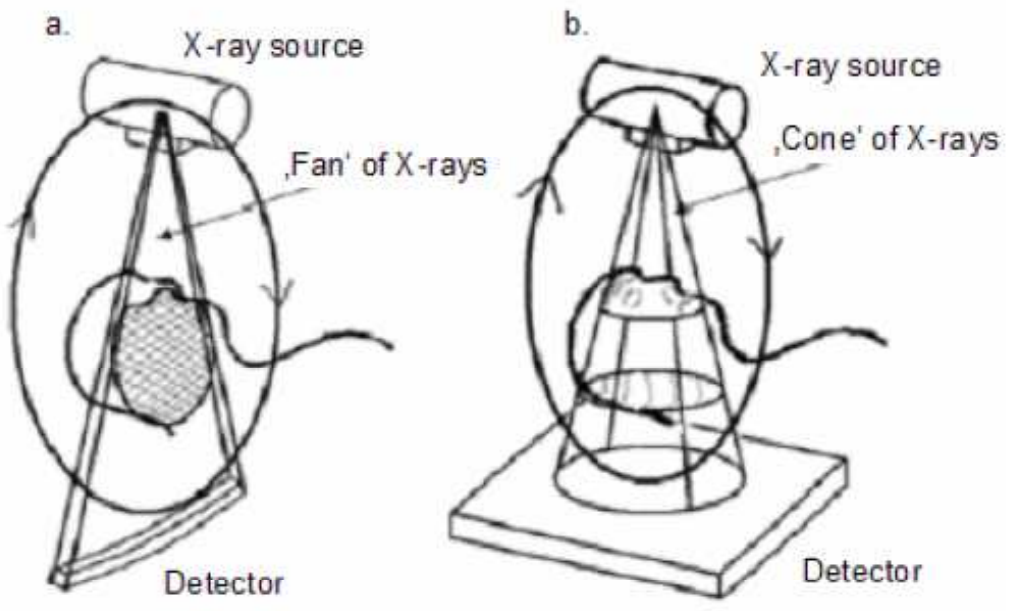

Abb. 2: Projektionsschema eines Multi-slice CT (a) im Vergleich zur Cone-beam-Technik eines DVT-Gerätes (b). Entnommen aus Scarfe et al. (2006) Seite 77. 


\subsection{Ziele der Arbeit}

Auf der Basis vorhandener DVT-Datensätze, die aufgrund verschiedener Indikationsstellungen unabhängig von einem OSAS-Verdacht aufgenommen wurden, sollen der Luftweg mit angrenzenden Strukturen und der Funktionszustand des orofazialen Systems untersucht werden.

Daraus sollen Schlüsselparameter entnommen werden, aufgrund derer die Wahrscheinlichkeit eines obstruktiven Schlafapnoe-Syndroms abgeleitet werden kann.

Teilfragestellung 1: Vermessung der Dimensionen des oberen (pharyngealen) Luftweges in der Sagittalen und in der Transversalen, sowie angrenzender Strukturen.

Teilfragestellung 2: Es soll der Funktionszustand des orofazialen Systems während der Aufnahme im DVT charakterisiert werden.

Teilfragestellung 3: Korrelation der Ergebnisse der Fragestellung 1 und 2 mit Angaben des verwendeten Fragebogens zum Auftreten und Schweregrad von Schlafapnoe und Rhonchopathien.

Teilfragestellung 4: Die Eignung eines DVT-Datensatzes zur Prognose von OSAS und Rhonchopathien anhand der Identifizierung von Risikofaktoren soll ermittelt werden. 


\section{Material und Methode}

\subsection{Patientenkollektiv}

Aus den ab 2006 bis Anfang 2009 vorhandenen DVT-Datensätzen wurden konsekutiv 104 Datensätze ausgewählt. Das Patientenkollektiv umfasste 104 Patienten im Alter von 30 bis 68 Jahren, das durchschnittliche Alter betrug 46,6 Jahre \pm 11,28 Stdabw.. Die Patienten waren beiderlei Geschlechts (52 Männer mit einen Durchschnittsalter von 46,82 Jahre \pm 11,25 Stdabw. und 52 Frauen mit einem Durchschnittsalter von 46,48 Jahre \pm 11,32 Stdabw.) mit ausgefülltem Schlaffragebogen. Die DVT-Aufnahmen wurden in der Universitätsklinik Göttingen, Abteilung Mund-, Kiefer- und Gesichtschirurgie mit dem digitalen Volumentomographen der Firma NewTom QR-9000 (Quantitative Radiology, Verona, Italien) erstellt. Die Patienten wurden im wachen und liegenden Zustand, mit geschlossenem Mund, ruhig durch die Nase atmend und mit habitueller Interkuspidation geröntgt. Die DVTAufnahmen wurden unabhängig von einem OSAS-Verdacht erstellt. Die Indikationen für diese Aufnahmen waren vor allem die prächirurgischen Abklärungen bei Zysten, Kieferhöhlenveränderungen, Weisheitszahnextraktionen oder zur präimplantatorischen Planung. Einschlusskriterien waren vollständig dargestellter Bereich vom harten Gaumen bis zur Vallecula oder bis zum Hyoid, posterior musste der Pharynx vollständig und anterior das Menton erkennbar sein. Es wurden nur Datensätze ausgewählt, bei denen der Patient zum Zeitpunkt der Aufnahme zwischen 30 und 70 Jahre alt war. Ausschlusskriterien waren Aufnahmen mit Bewegungsartefakten, keine Okklusion während der Aufnahme und keine vorhandenen Frontzähne.

\subsection{Fragebogenanalyse}

Von den Patienten wurde ein Fragebogen aus der Schnarchsprechstunde der Universität Göttingen ausgefüllt. Dieser beinhaltet Fragen zu vorangegangen Untersuchungen in Bezug auf obstruktive Schlafapnoe, Alter, Größe, Gewicht und zum Schlaf- und Schnarchverhalten sowie die Epworth Sleepiness Scale (ESS).

In der Epworth Sleepiness Scale nach Johns (1993) wird erfragt, wie wahrscheinlich man in acht verschiedenen Situationen, z.B. beim Sitzen und Lesen, beim Fernsehen, als Beifahrer im Auto oder beim Besuch öffentlicher Veranstaltungen, einschlafen würde. Die Bewertung erfolgt mit Hilfe einer Punkteskala, die Häufigkeitsangaben entsprechen von „nie“ (0) bis 
„oft“ (3). Die Gesamtpunktzahl kann minimal bei 0 und maximal bei 24 liegen. Die Skala gibt nur die Einschlafneigung, aber nicht die Häufigkeit und die Schlafdauer an.

Die Schnarchlautstärke wurde von dem Partner auf einer visuellen Analogskala von 1 bis 10 beurteilt, wobei 1 eine niedrige Lautstärke und 10 eine hohe Lautstärke bedeutet.

Die Häufigkeit des Schnarchens wurde in einer visuellen Analogskala von 1 bis 5 eingeteilt. Dabei bedeutet 1 „fast nie“, 2 ,1-2 mal pro Monat“, 3 „1-2 mal pro Woche“, 4 , , 3-4 mal pro Woche“ und 5 , fast jeden Tag“.

\subsection{Radiologische Befunderhebung}

\subsubsection{Gerät und Einstellung}

Bei dem verwendeten digitalen Volumentomographen NewTom QR-9000 (Quantitative Radiology, Verona, Italien) (Abb. 3) besteht der Detektor aus einem Bildverstärker (BV) der Firma Thomson mit einem BV-Eingangsfenster von 8x8 Zoll, der Verstärkungsfaktor beträgt 22:1. Die Bilderfassung erfolgt mit Hilfe eines $\mathrm{CCD}(\mathrm{CCD}=$ charge-couple-device) -Chips mit einer Matrix von 752x582 Pixel und die Bearbeitung des bis zu 94 Megabyte großen Rohdatensatzes erfolgte mit der Rekonstruktionssoftware NewTom 9000 Version 3.37.

Bei einem lateralen und anterior-posterioren Probescan werden die Dichtewerte der Hartgewebe gemessen und die Einstellparameter für die Belichtungsautomatik berechnet. Durch diese sogenannte „smart-beam-Technik“ wird eine Dosisreduktion erzielt, das Gerät misst hierbei selbstständig die kleinste ausreichende Strahlenexposition zur adäquaten Durchleuchtung des Objektes. Der Röhrenstrom variiert je nach Knochenvolumen und -dichte (Mozzo et al. 1998). Die Röntgenröhre arbeitet mit mittelharter Strahlung, d.h. es wird eine konstante Spannung von $110 \mathrm{kV}$ verwendet. Die Filterung erfolgt mit 7,2 mm Aluminium bei einem konstanten Öffnungswinkel von 14 Grad. Bei der Aufnahme wird ein Zylinder von 15 cm Höhe und $15 \mathrm{~cm}$ im Durchmesser abgebildet. Die gesamte Aufnahmezeit beträgt 76 Sekunden, aber die tatsächliche Zeit der Strahlenexposition beträgt aufgrund des gepulsten Röntgenstrahls nur 18 Sekunden.

Anschließend wurde aus den 360 einzelnen Projektionsradiographien ein dreidimensionaler Datensatz rekonstruiert. Nach der Aufnahme wurde anhand eines Planungstomogramms die axiale Primärrekonstruktion festgelegt, die in ihrer vertikalen Lage und ihrem sagittalen Winkel zur Frankfurter Horizontalen frei wählbar ist, um eine für den individuellen Fall optimale Diagnostik zu ermöglichen. Die Dicke der axialen Schicht kann zwischen 0,3 mm, 1 
mm und $2 \mathrm{~mm}$, der maximale Durchmesser der axialen Schnitte zwischen $128 \mathrm{~mm}$ (small field) und $148 \mathrm{~mm}$ (large field) variiert werden. Mit dem $148 \mathrm{~mm}$ umfassenden Durchmesser der axialen Schnitte ist es bei entsprechender Positionierung des Patienten möglich, den gesamten für die Zahn-, Mund- und Kieferheilkunde relevanten Bereich darzustellen. Die Voxelgröße beträgt 0,25 (1) (2) x 0,25 (0,29) x 0,25 (0,29) $\mathrm{mm}$, wobei wahlweise mit den eingeklammerten Werten gearbeitet werden kann. Es lassen sich beliebig viele Primärrekonstruktionen aus einem Rohdatensatz erstellen, da dieser archiviert wird.

Aus der Primärrekonstruktion können Sekundärrekonstruktionen in sagittalen, koronalen, paraaxialen Schichten, sowie in Panoramaübersichten und 3D-Ansichten erstellt werden (Möbes et al. 2000, Mozzo et al. 1998). Mit der verwendeten NewTom 9000 Software (Version 3.37) ist es möglich, in den konstruierten Schnitten Strecken und Winkel zu messen, sowie Hilfsmarker zu setzen. Die in diesem Programm angegebenen Werte entsprechen den anatomischen Strecken im Maßstab 1:1. Es können 0,5 mm, 1,5 mm oder 3,0 mm große farbige Marker verwendet werden. Die automatische Übertragung der Farbmarkierungen in alle Schnitte ermöglicht eine Vermessung von Distanzen zwischen den einzelnen Schnitten.

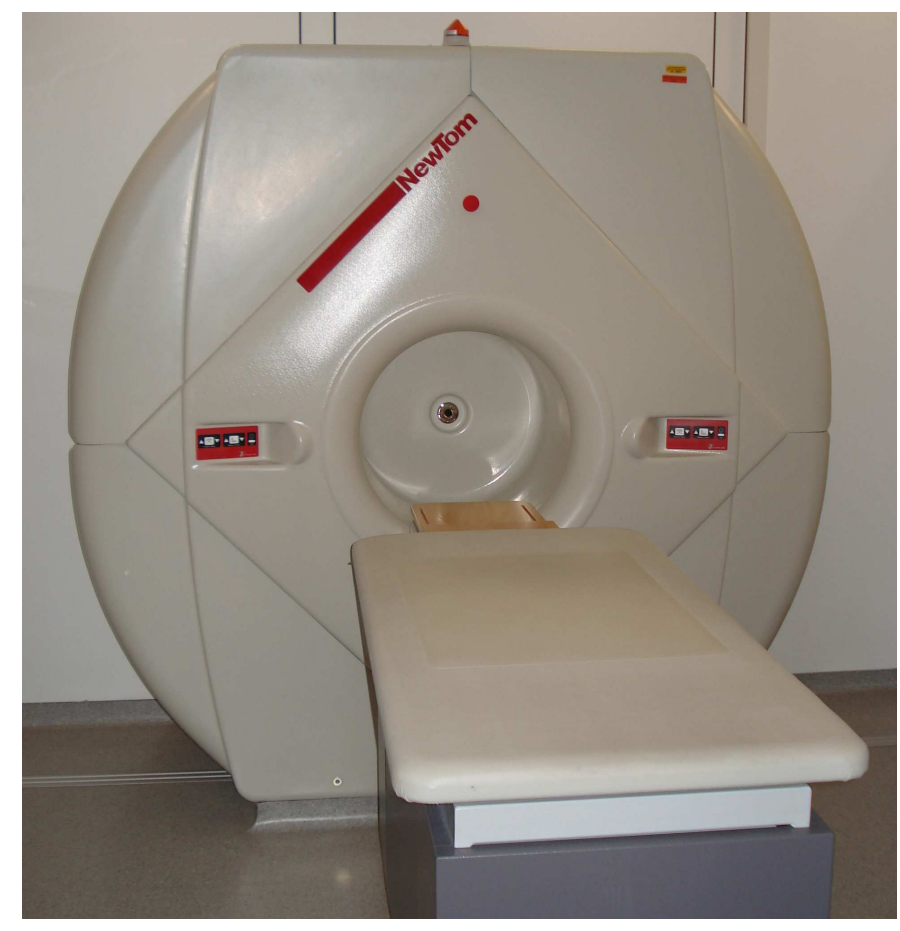

Abb. 3: DVT NewTom QR-9000 


\subsubsection{Analyse der Datensätze}

Die Auswertung der Datensätze erfolgte an dem in der Universitätsklinik Göttingen, Abteilung Mund-, Kiefer- und Gesichtschirurgie, verwendeten Befundsmonitor KAT.B nach DIN 6868-57 U. QSRL des Herstellers EIZO und mit der NewTom 9000 Software Version 3.37 .

Aus dem Rohdatensatz wurde rechnergestützt ein dreidimensionaler Datensatz rekonstruiert. Die primäre axiale Rekonstruktion wurde parallel der harten Gaumenebene (PNS-ANS) ausgerichtet und erstreckte sich von oberhalb des harten Gaumens bis zum Hyoid. Bei der Primärrekonstruktion wurden folgende Einstellungen gewählt: „Large field“ und $1 \mathrm{~mm}$ dicke axiale Schnitte. Für die Sekundärrekonstruktionen wurden ein mediosagittaler Schnitt orientiert an ANS und PNS, ein koronaler Schnitt auf der Hälfte der ANS-PNS-Strecke und ein Schnitt von Gonion zu Menton berechnet.

In dem mediosagittalen Schnitt (Abb. 4 und 5) wurde die Länge des harten Gaumens (ANSPNS), die Kontaktfläche von der Zunge zum weichen Gaumen, die Länge des weichen Gaumens (PNS-Uvulaspitze), die Dicke des weichen Gaumens an der breitesten Stelle, die Höhe des kollapsfähigen Segmentes (PNS-Epiglottisspitze), der kürzester Abstand vom Hyoid senkrecht zur harten Gaumenebene, die Strecke zwischen ANS und dem Schnittpunkt von der harten Gaumenebene mit der Hyoidsenkrechten und die Lufthöhe unter dem harten Gaumen gemessen. 


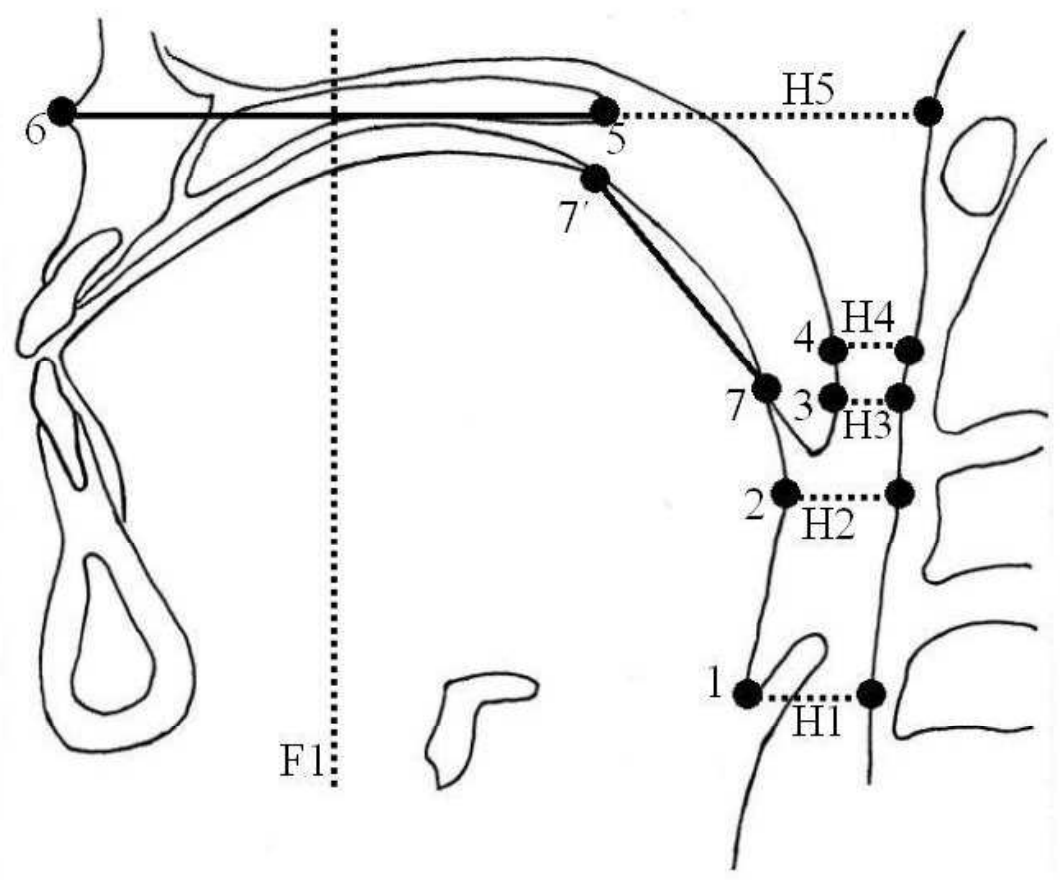

Abb. 4: Messschema mediosagittaler Schnitt (1)

$1 \quad$ Vallecula

2 dorsalster Punkt des Zungenrückens

3 vordere Begrenzung des Luftweges auf Höhe des unteren Inzisialpunktes

4 anteriorer Punkt auf Höhe Isthmus

$5 \quad$ Spina nasalis posterior (PNS)

$6 \quad$ Spina nasalis anterior (ANS)

7 kaudale Begrenzung der Kontaktfläche der Zunge mit dem weichen Gaumen

7' kraniale Begrenzung der Kontaktfläche der Zunge mit dem weichen Gaumen

Strecke 5-6: $\quad$ Länge harter Gaumen (ANS-PNS)

Strecke 7-7’: $\quad$ Kontaktstrecke weicher Gaumen mit Zunge

F1: $\quad$ koronale Schnittebene auf Höhe der Hälfte des Abstandes zwischen Spina nasalis anterior und Spina nasalis posterior

H1: $\quad$ axiale Schnittebene auf Höhe der Vallecula parallel zur Nasallinie (LPW)

H2: $\quad$ axiale Schnittebene auf Höhe des dorsalsten Punktes des Zungenrückens parallel zur Nasallinie (TPW)

H3: axiale Schnittebene auf Höhe des unteren Inzisialpunktes parallel zur Nasallinie (MPW)

H4: axiale Schnittebene auf Höhe Isthmus parallel zur Nasallinie (Isthmus)

H5: axiale Schnittebene auf Höhe der Nasallinie (UPW) 


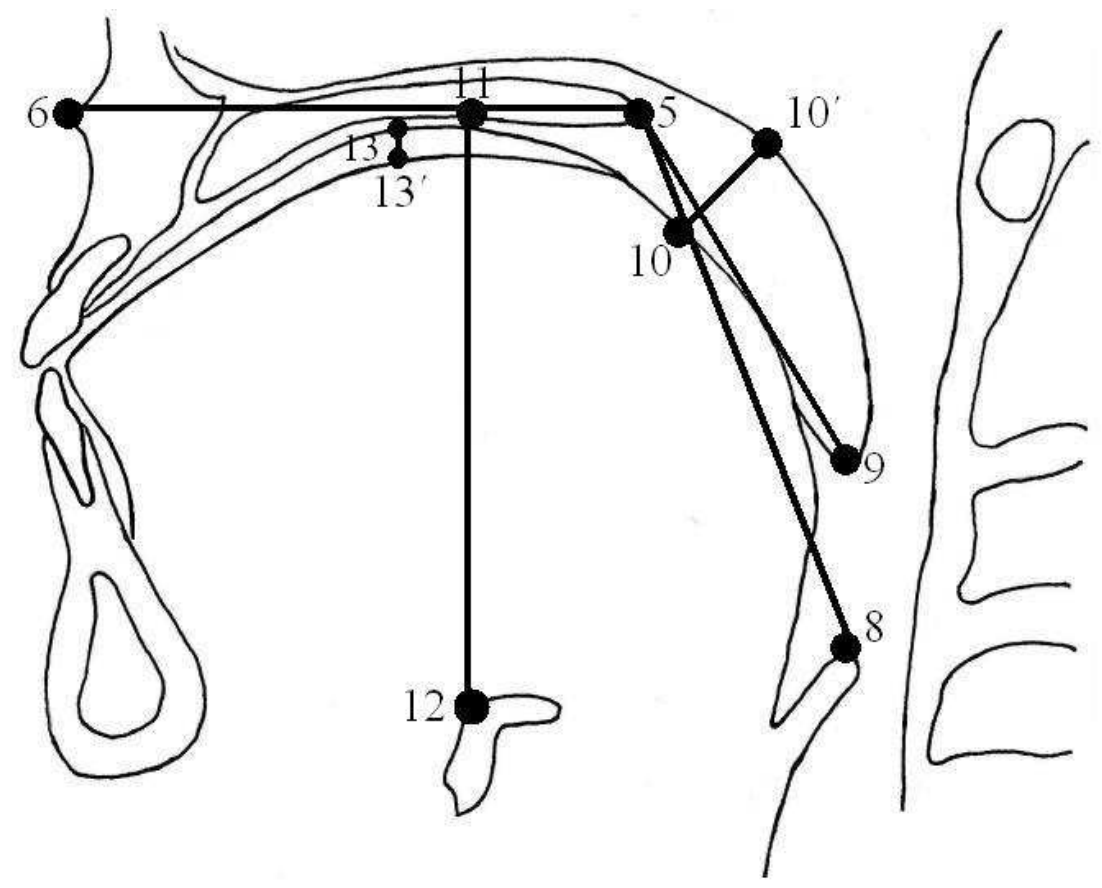

Abb. 5: Messschema mediosagittaler Schnitt (2)

$5 \quad$ Spina nasalis posterior (PNS)

6 Spina nasalis anterior (ANS)

9 Uvulaspitze

$8 \quad$ Epiglottisspitze

10 Punkt auf der oralen Fläche, an dem der weiche Gaumen am dicksten ist

10' Punkt auf der nasalen Fläche, an dem der weiche Gaumen am dicksten ist

11 Schnittpunkt der Hyoidsenkrechten mit der Nasallinie

12 vorderster oberster Punkt des Hyoids

13 obere Begrenzung der Lufthöhe unter dem harten Gaumen

13 untere Begrenzung der Lufthöhe unter dem harten Gaumen

Strecke 5-8: Höhe des kollapsfähigen Segmentes (PNS-Epiglottis)

Strecke 5-9: Länge des weichen Gaumens

Strecke 6-11: Abstand zwischen Spina nasalis anterior und dem Schnittpunkt von der Nasallinie mit der Hyoidsenkrechten (ANS-Hyoidsenkrechten)

Strecke 10-10’: Dicke des weichen Gaumens an der breitesten Stelle

Strecke 11-12: $\quad$ kürzester Abstand vom Hyoid senkrecht zur Nasallinie (Hyoid-Nasallinie)

Strecke 13-13’: $\quad$ Lufthöhe unter dem harten Gaumen 
In dem koronalen Schnitt (Abb. 6) wurde die Strecke zwischen dem harten Gaumen und der Verbindungslinie der rechten und linken Unterkieferspange vermessen. Weiterhin wurde der Abstand zwischen den lingualen Höckern der Unterkieferzähne ermittelt. Außerdem wurde die palatinale Höhe zwischen der Verbindungslinie der lingualen Unterkieferhöcker und der Mitte des harten Gaumens gemessen.

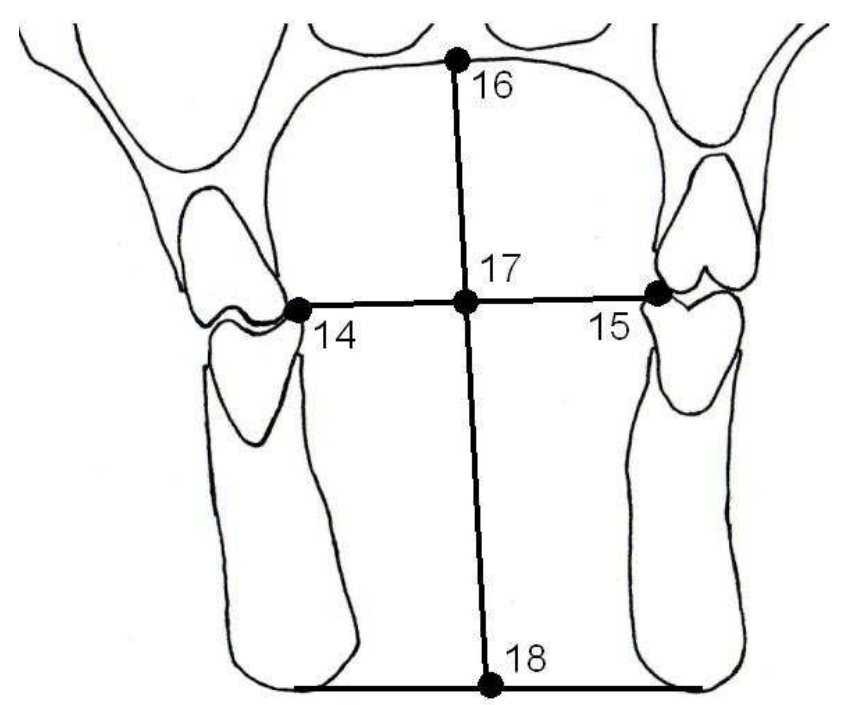

Abb. 6: Messschema koronaler Schnitt

14 linguale Höckerspitze des rechten unteren Sechsjahresmolaren

15 linguale Höckerspitze des linken unteren Sechsjahresmolaren

16 harter Gaumen in der Mediosagittalen

17 Schnittpunkt der Mediosagittalen mit der Verbindungslinie zwischen den lingualen Höckerspitzen der unteren Sechsjahresmolaren

18 Schnittpunkt der Mediosagittalen mit der Verbindungslinie der unteren Kontur des UK

Strecke 14-15: Abstand der lingualen Höckerspitzen der unteren Sechsjahresmolaren

Strecke 16-17: Abstand des harten Gaumens zur Verbindungslinie zwischen den lingualen Höckerspitzen der unteren Sechsjahresmolaren (palatinale Höhe)

Strecke 16-18: Abstand der Nasallinie zur Verbindungslinie der Unterkieferspange (NL-UK)

In den parallel zur harten Gaumenebene ausgerichteten axialen Schnitten wurde der Luftweg auf Höhe der Vallecula (LPW), des dorsalsten Punktes der Zunge (TPW), des unteren Inzisialpunktes (MPW) (Abb. 7), der engsten Stelle des Luftweges (Isthmus) und der harten Gaumenebene (UPW) in der transversalen und sagittalen Richtung im Bereich der größten Ausdehnung vermessen. Dabei wurde darauf geachtet, dass die transversalen und sagittalen Verbindungsstrecken senkrecht zueinander orientiert sind. Der Isthmus wurde definiert, als die Stelle, an dem der Luftweg die geringste Fläche aufweist. Bei zweigeteilten Luftwegen wurden die beiden Öffnungsquerschnitte separat nach den oben beschriebenen Kriterien 
vermessen. Die Fläche des Luftweges wurde als Ellipse angenommen und mit der Formel A = $\pi \times \mathrm{x} / 2 \mathrm{x}$ b/2 berechnet. Ferner wurde in den axialen Schnitten beurteilt, ob Funktionskompartiment B (palatolinguales Kompartiment) und C (mesopharyngeales Kompartiment) voneinander getrennt sind. Stehen die Kompartimente B und C in Verbindung, liegt ein offener orofazialer Systemzustand vor. Bei getrennten Kompartimenten besteht ein geschlossener orofazialer Systemzustand.

Zusätzlich wurden auf Höhe des unteren Inzisialpunktes die intermaxilläre Raumlänge (Abstand Unterkieferinzisivi zur dorsalen Pharynxwand) und die Winkel zwischen der Innenkortikalis bzw. Außenkortikalis an der Vorderkante des linken aufsteigenden Unterkieferastes und der Verbindungslinie der distalen Konturen des Unterkiefers gemessen.

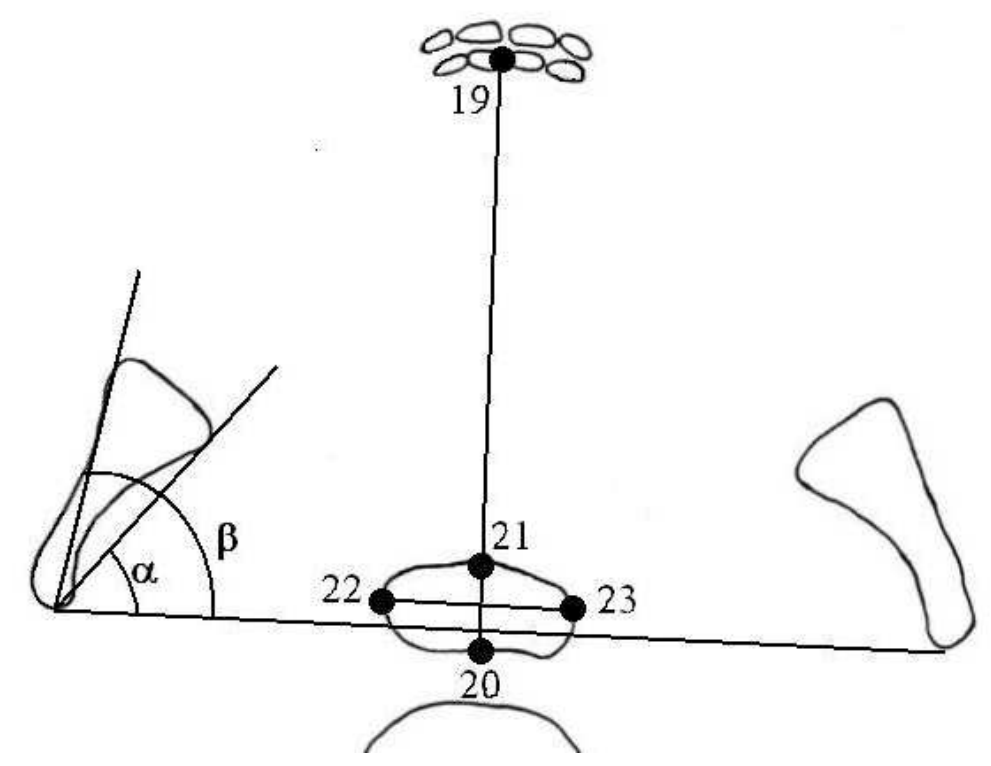

Abb. 7: Messschema axialer Schnitt auf Höhe MPW

19 unterer Inzisialpunkt

20 hintere Pharynxwand auf Höhe des unteren Inzisialpunktes

21 vordere Begrenzung des Luftweges auf Höhe des unteren Inzisialpunktes

22 transversale Begrenzung des Luftweges auf Höhe des unteren Inzisialpunktes links

23 transversale Begrenzung des Luftweges auf Höhe des unteren Inzisialpunktes rechts

Strecke 19-20: intermaxilläre Raumlänge

Strecke 20-21: $\quad$ sagittale Ausdehnung des Luftweges auf Höhe des unteren Inzisialpunktes

Strecke 22-23: transversale Ausdehnung des Luftweges auf Höhe des unteren Inzisialpunktes

$\alpha \quad$ Innenwinnkel: Winkel zwischen Innenkortikalis der Vorderkante des aufsteigenden Unterkieferastes und der Verbindungslinie der distalen Konturen des Unterkiefers

$\beta \quad$ Außenwinkel: Winkel zwischen Außenkortikalis der Vorderkante des aufsteigenden Unterkieferastes und der Verbindungslinie der distalen Konturen des Unterkiefers 
Die Unterkieferlänge wurde in einer Schnittebene, die durch das Gonion und Menton verläuft, gemessen (Abb. 8).

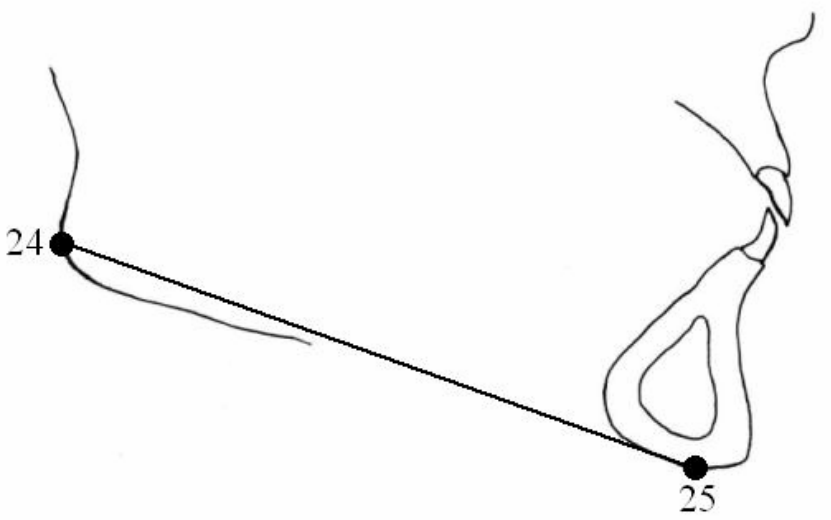

Abb. 8: Messschema für Unterkieferlänge

24 Gonion

25 Menton

Strecke 24-25: Unterkieferlänge 


\subsection{Statistische Auswertung}

Die statistische Auswertung der vorliegenden Arbeit wurde mit dem Programm Statistica V 8 (FA. Statsoft, Tusla, USA) durchgeführt.

Metrische Werte wurden mit dem Alter und dem BMI unter Verwendung von Korrelationsmatrizen nach Pearson untereinander verglichen, da stetige Messwerte vorlagen und eine Normalverteilung angenommen werden konnte.

Für die Korrelation nichtparametrischer Werte (ESS, Häufigkeit und Lautstärke des Schnarchens) mit metrischen Werten wurde der Spearman-Test verwendet.

Der Vergleich zweier unabhängiger Stichproben (Vergleich System offen/geschlossen mit metrischen Daten) wurde mit dem Mann-Whitney-U-Test durchgeführt.

Zur graphischen Darstellung der Werte wurden Box-Plots und Histogramme benutzt.

Es wurde für die statistischen Auswertungen ein Signifikanzniveau von 5\% festgelegt, somit gelten Ergebnisse mit p-Werten kleiner als 0,05 als signifikant. Die p-Werte wurden bis zur dritten Stelle nach dem Komma angegeben, bei Werten kleiner 0,001 wurde im Verlauf der Arbeit $\mathrm{p}<0,001$ geschrieben.

\subsection{Ethikkommissionsvotum}

Die Ethikkommission der Medizinischen Fakultät hatte hinsichtlich der Durchführung dieser Untersuchung keine Bedenken (Antragsnummer: 7/6/08). 


\section{Ergebnisse}

\subsection{Ergebnisse der Patientenbefragung}

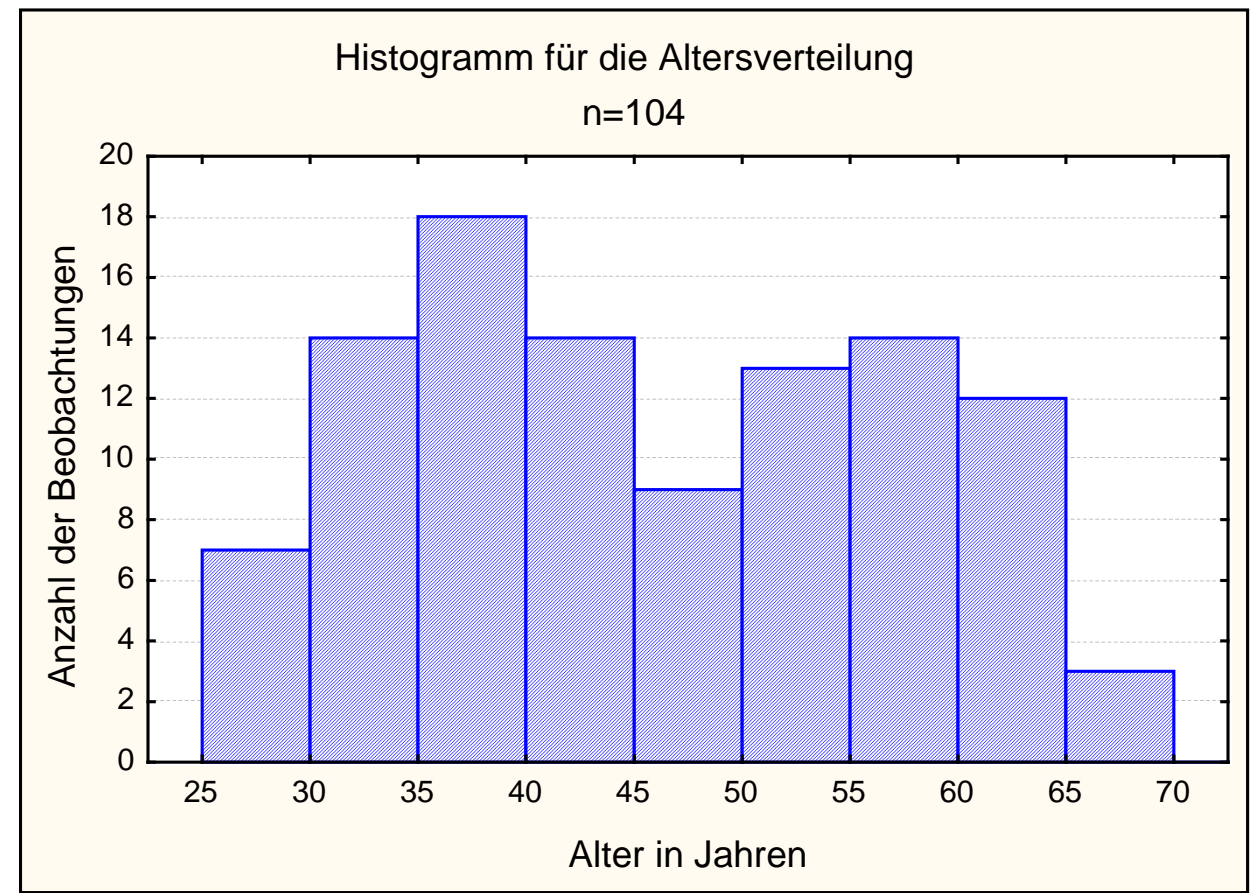

Abb. 9 : Histogramm für die Altersverteilung

Das Histogramm zeigt die Verteilung der Altersgruppen innerhalb des Patientenkollektives.

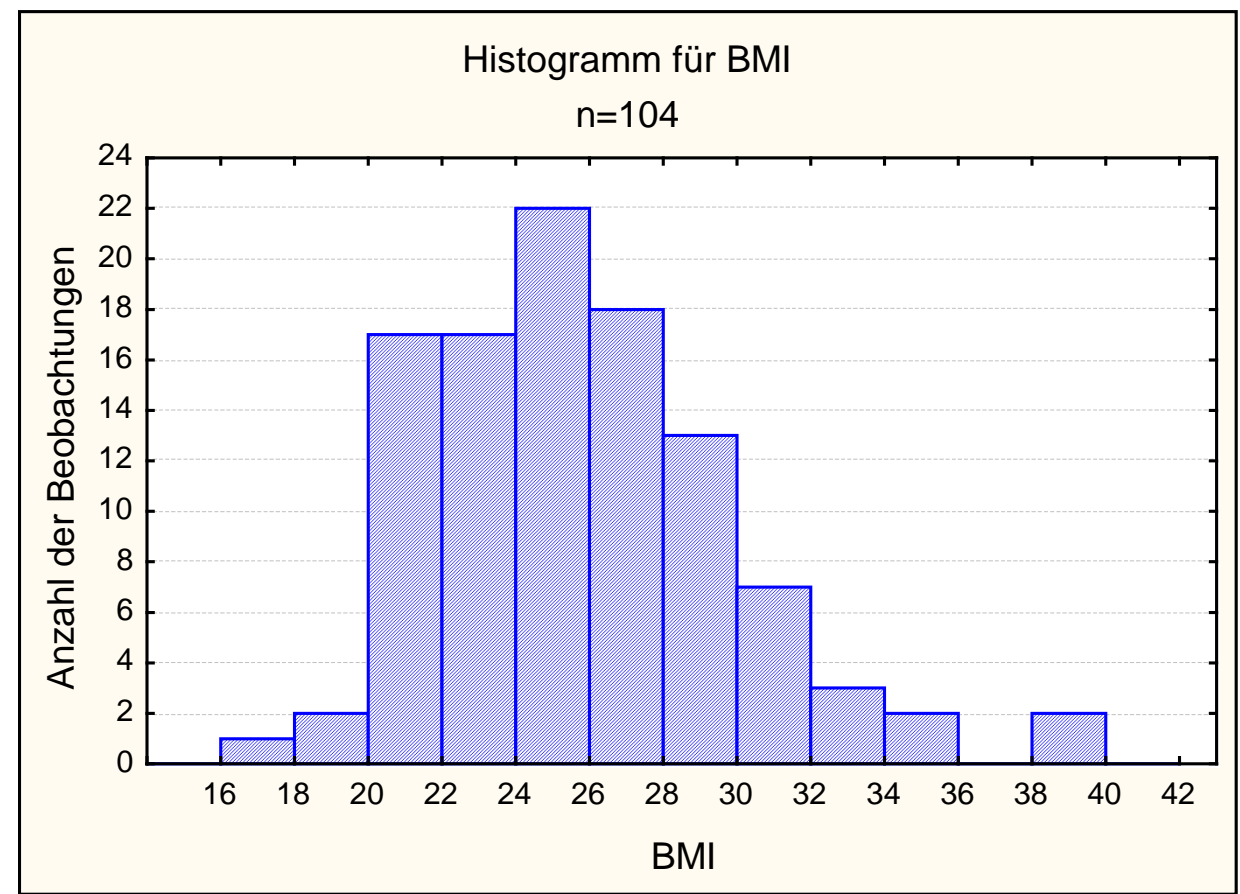

Abb. 10 : Histogramm für den BMI

Das Histogramm zeigt die Verteilung des BMI innerhalb des Patientenkollektives. 


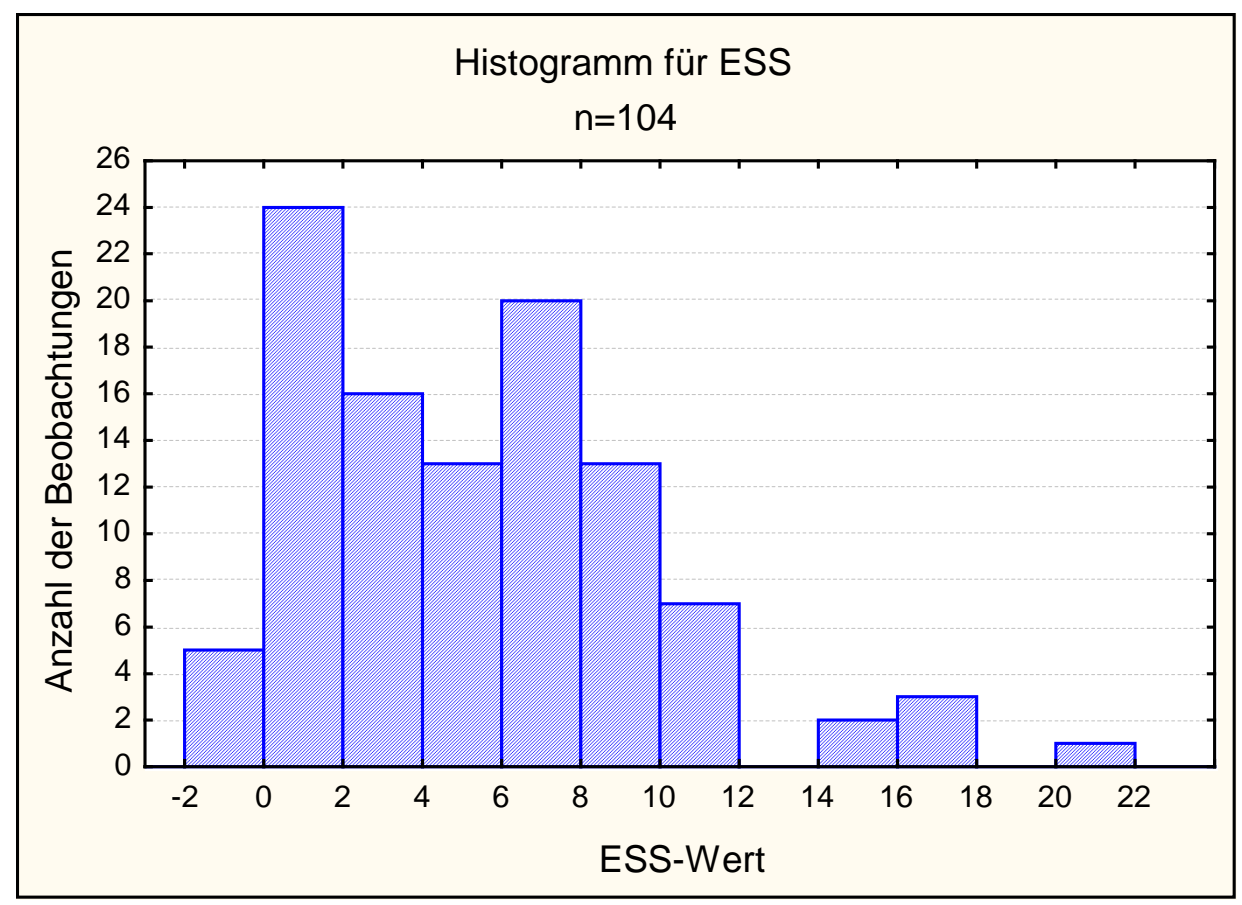

Abb. 11 : Histogramm für den ESS-Wert

Das Histogramm zeigt die Verteilung der ESS-Werte innerhalb des Patientenkollektives.

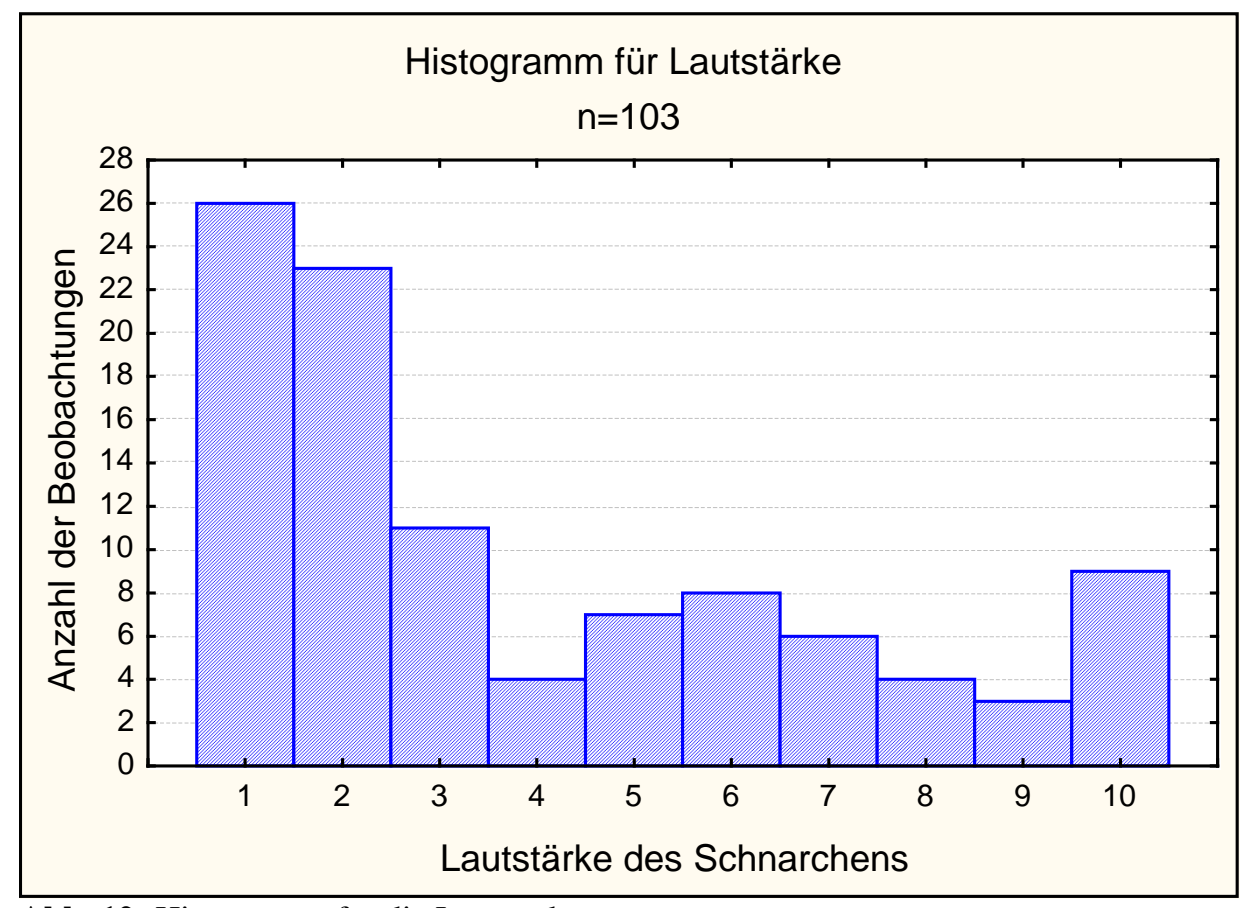

Abb. 12: Histogramm für die Lautstärke

Das Histogramm zeigt die Verteilung der Lautstärke des Schnarchens innerhalb des Patientenkollektives. Die Schnarchlautstärke wurde von dem Partner auf einer Skala von 1 bis 10 beurteilt, wobei 1 eine niedrige Lautstärke und 10 eine hohe Lautstärke bedeutet. 


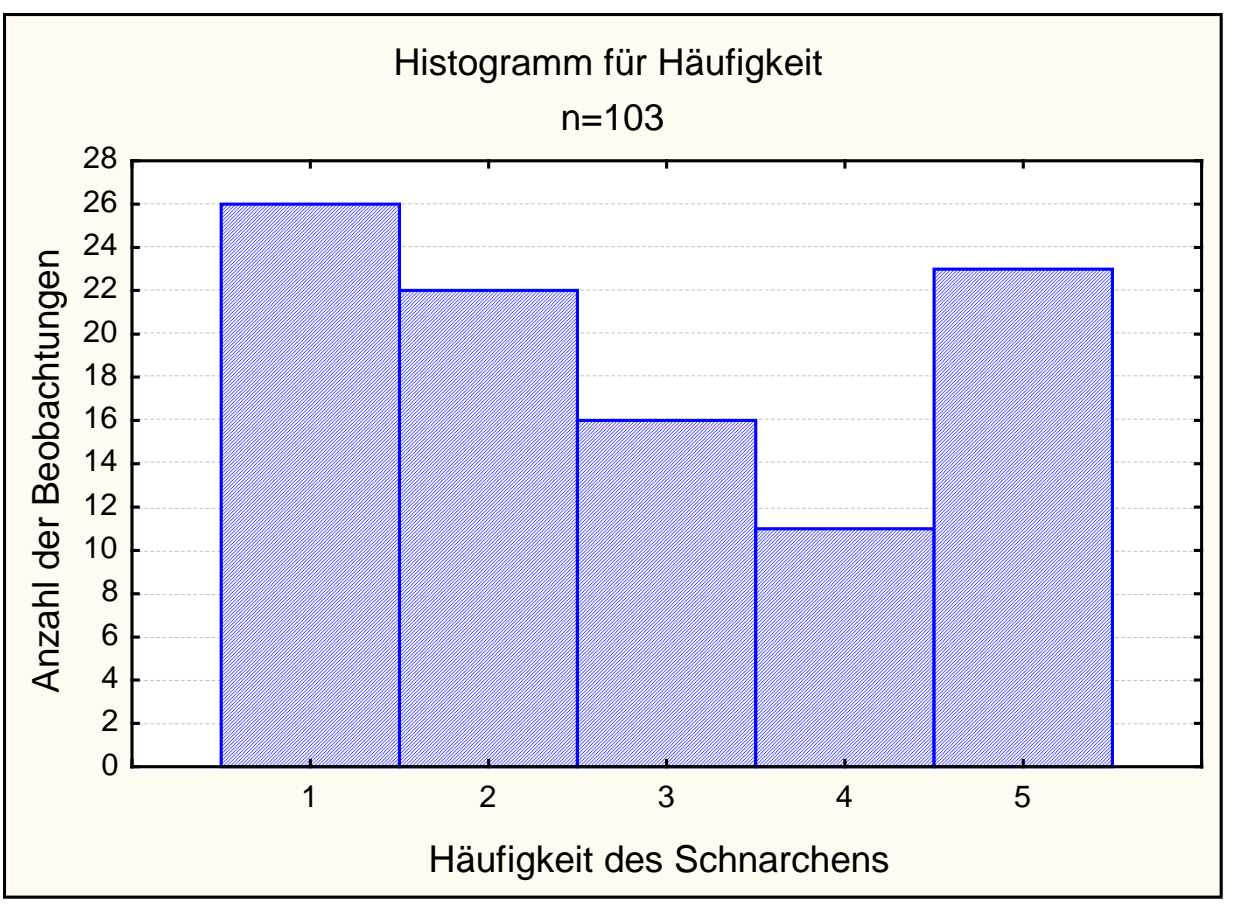

Abb. 13: Histogramm für die Häufigkeit

Das Histogramm zeigt die Verteilung der Häufigkeit des Schnarchens innerhalb des Patientenkollektives. Die Häufigkeit des Schnarchens wurde in einer Skala von 1 bis 5 eingeteilt. Dabei bedeutet 1 „fast nie“, 2 „,1-2 mal pro Monat“, 3 „,1-2 mal pro Woche“, 4 ,, 34 mal pro Woche“ und 5 ,, fast jeden Tag“.

\subsection{Deskriptive Darstellung der Messungen}

Die folgende Tabelle 2 zeigt die Mittelwerte und deren Standardabweichungen für die verschiedenen Patientengruppen. In der ersten Spalte sind Patienten mit ESS-Werten $\geq 9$ und mit bestätigtem OSAS, in der zweiten Spalte sind Patienten mit bestätigtem OSAS und in der dritten Spalte sind alle 104 Patienten zugrunde gelegt worden. 
ESS $\geq 9$ und

OSAS bestätigt

Mittelwert Stdabw. $\mathrm{n}$

\begin{tabular}{|c|c|c|c|}
\hline & & & \\
\hline ANS-PNS (mm) & 55,04 & 3,63 & 29 \\
\hline Länge weicher Gaumen (mm) & 39,51 & 6,50 & 29 \\
\hline Dicke weicher Gaumen (mm) & 10,52 & 2,03 & 18 \\
\hline PNS-Epiglottis (mm) & 57,19 & 10,32 & 29 \\
\hline Kontaktstrecke $(\mathrm{mm})$ & 18,14 & 15,32 & 29 \\
\hline Hyoid-Nasallinie $(\mathrm{mm})$ & 61,92 & 9,41 & 19 \\
\hline ANS-Hyoidsenkrechte $(\mathrm{mm})$ & 56,15 & 5,76 & 19 \\
\hline Lufthöhe (mm) & 2,91 & 3,84 & 29 \\
\hline UPW anterior-posterior (mm) & 17,60 & 3,61 & 29 \\
\hline UPW transversal (mm) & 23,91 & 4,58 & 29 \\
\hline UPW Fläche $\left(\mathrm{mm}^{2}\right)$ & 336,39 & 117,19 & 29 \\
\hline MPW anterior-posterior $(\mathrm{mm})$ & 8,64 & 2,66 & 28 \\
\hline MPW transversal (mm) & 19,38 & 6,79 & 28 \\
\hline MPW Fläche $\left(\mathrm{mm}^{2}\right)$ & 133,44 & 74,35 & 29 \\
\hline Intermaxilläre Raumlänge (mm) & 77,94 & 5,49 & 28 \\
\hline Innenwinkel (9) & 51,50 & 4,41 & 28 \\
\hline Außenwinkel ( 9 & 76,15 & 4,20 & 28 \\
\hline LPW anterior-posterior $(\mathrm{mm})$ & 11,64 & 2,52 & 14 \\
\hline LPW transversal (mm) & 29,73 & 4,99 & 14 \\
\hline LPW Fläche $\left(\mathrm{mm}^{2}\right)$ & 269,92 & 74,26 & 15 \\
\hline Isthmus anterior-posterior (mm) & 6,18 & 1,86 & 26 \\
\hline Isthmus transversal (mm) & 17,35 & 6,22 & 26 \\
\hline Isthmus Fläche $\left(\mathrm{mm}^{2}\right)$ & 85,89 & 46,59 & 29 \\
\hline Abstand Isthmus-Nasallinie (mm) & 31,48 & 9,69 & 29 \\
\hline TPW anterior-posterior ( $\mathrm{mm}$ ) & 10,56 & 3,68 & 29 \\
\hline TPW transversal (mm) & 24,16 & 5,75 & 29 \\
\hline TPW Fläche $\left(\mathrm{mm}^{2}\right)$ & 208,02 & 108,34 & 29 \\
\hline Nasallinie-Unterkiefer (mm) & 59,73 & 6,39 & 29 \\
\hline Abstand linguale Höcker (mm) & 34,70 & 4,23 & 29 \\
\hline palatinale Höhe (mm) & 22,24 & 3,50 & 29 \\
\hline Unterkieferlänge (mm) & 86,35 & 4,97 & 29 \\
\hline Alter & 47,76 & 10,77 & 29 \\
\hline BMI & 27,22 & 4,97 & 29 \\
\hline
\end{tabular}

Tab. 2: Mittelwerte
OSAS bestätigt Gesamtkollektiv

Mittelwert Stdabw. $\mathrm{n}$ Mittelwet Stdabw. $\mathrm{n}$

\begin{tabular}{|c|c|c|c|c|c|}
\hline 5,93 & 3,71 & 8 & 54,74 & 3,46 & 104 \\
\hline 42,15 & 7,86 & 8 & 38,94 & 5,22 & 104 \\
\hline 11,96 & 3,10 & 5 & 9,99 & 1,82 & 58 \\
\hline 62,00 & 12,07 & 8 & 55,53 & 8,22 & 103 \\
\hline 18,53 & 18,31 & 8 & 19,70 & 14,84 & 104 \\
\hline 72,70 & 13,44 & 3 & 62,87 & 8,54 & 88 \\
\hline 53,13 & 7,26 & 3 & 55,04 & 8,21 & 88 \\
\hline 3,53 & 5,43 & 8 & 2,37 & 3,29 & 104 \\
\hline 17,89 & 4,43 & 8 & 18,25 & 3,75 & 104 \\
\hline 22,13 & 3,34 & 8 & 23,81 & 4,32 & 104 \\
\hline 320,31 & 119,69 & 8 & 343,24 & 117,13 & 104 \\
\hline 8,30 & 2,19 & 8 & 8,50 & 3,09 & 104 \\
\hline 16,14 & 8,08 & 8 & 18,91 & 6,51 & 104 \\
\hline 113,53 & 81,43 & 8 & 129,86 & 75,62 & 104 \\
\hline 79,06 & 4,86 & 8 & 78,19 & 5,81 & 103 \\
\hline 53,00 & 5,65 & 8 & 52,44 & 4,64 & 103 \\
\hline 76,58 & 4,36 & 8 & 77,44 & 3,75 & 103 \\
\hline 9,55 & 2,90 & 2 & 12,59 & 2,87 & 74 \\
\hline 26,00 & 7,21 & 2 & 29,76 & 7,57 & 74 \\
\hline 186 & 5,10 & 2 & 297,09 & 111,55 & 74 \\
\hline 7,13 & 2,16 & 7 & 6,41 & 1,98 & 100 \\
\hline 14,97 & 9,60 & 7 & 17,75 & 6,33 & 100 \\
\hline 93,35 & 72,26 & 8 & 92,22 & 54,23 & 104 \\
\hline 33,63 & 11,36 & 8 & 30,68 & 9,09 & 104 \\
\hline 10,60 & 5,05 & 8 & 10,82 & 3,28 & 104 \\
\hline 23,93 & 6,43 & 8 & 25,31 & 6,78 & 104 \\
\hline 209,25 & 148,64 & 8 & 221,08 & 103,46 & 104 \\
\hline 60,18 & 5,68 & 8 & 60,15 & 5,63 & 104 \\
\hline 35,66 & 4,76 & 8 & 34,77 & 4,29 & 104 \\
\hline 22,44 & 3,56 & 8 & 22,62 & 2,92 & 104 \\
\hline 87,85 & 5,65 & 8 & 86,11 & 5,11 & 103 \\
\hline 53,88 & 11,34 & 8 & 46,66 & 11,23 & 104 \\
\hline 0,96 & 5,45 & 8 & 25,72 & 4,20 & 104 \\
\hline
\end{tabular}




\begin{tabular}{|l|c|}
\hline OSAS-bestätigte Patienten & ESS-Werte \\
\hline $\mathrm{n}=8$ & 12 \\
\cline { 2 - 2 } & 1 \\
\cline { 2 - 2 } & 9 \\
\cline { 2 - 2 } & 12 \\
\cline { 2 - 2 } & 9 \\
\cline { 2 - 2 } & 2 \\
\cline { 2 - 2 } & 5 \\
\cline { 2 - 2 } & 16 \\
\hline
\end{tabular}

Tab. 3: ESS-Werte der Patienten mit bestätigtem OSAS

Die Tabelle 3 führt die ESS-Werte von Patienten mit bestätigtem OSAS auf, dabei zeigt sich eine große Streubreite und teils niedrige ESS-Werte.

\subsection{Korrelation von Fragebogenergebnissen und radiologischen Messwerten}

\subsubsection{Alter}

Der Zusammenhang zwischen dem Alter und den Messwerten wurde mithilfe von Korrelationsmatrizen nach Pearson ermittelt. Eine signifikante Korrelation wurde ab einem pWert $<0,05$ angenommen.

\begin{tabular}{|l|l|l|l|}
\hline \multicolumn{3}{|c|}{ Korrelationsmatrizen nach Pearson } & \multicolumn{3}{c|}{$\begin{array}{r}\text { markierte Korrelation } \\
\text { signifikant ab p } 0,050\end{array}$} \\
\hline Variable \& ANS-PNS & $\mathrm{N}$ & \multicolumn{3}{|c|}{ p-Niveau } \\
\hline Alter & 104 & $-0,122$ & 0,449 \\
\hline Alter \& Länge weicher Gaumen & 104 & 0,454 & 0,003 \\
\hline Alter \& Dicke weicher Gaumen & 58 & 0,203 & 0,202 \\
\hline Alter \& PNS-Epiglottis & 103 & 0,286 & 0,070 \\
\hline Alter \& Kontaktstrecke & 104 & $-0,113$ & 0,480 \\
\hline Alter \& Hyoid-Nasallinie & 88 & 0,406 & 0,008 \\
\hline Alter \& ANS-Hyoidsenkrechten & 88 & $-0,071$ & 0,509 \\
\hline Alter \& Lufthöhe & 104 & 0,025 & 0,875 \\
\hline Alter \& UPW anterior-posterior & 103 & 0,117 & 0,466 \\
\hline Alter \& UPW transversal & 103 & 0,144 & 0,368 \\
\hline Alter \& UPW Fläche & 103 & 0,150 & 0,203 \\
\hline Alter \& MPW anterior-posterior & 102 & $-0,478$ & 0,002 \\
\hline Alter \& MPW transversal & 102 & 0,091 & 0,574 \\
\hline Alter \& MPW Fläche & 104 & $-0,103$ & 0,385 \\
\hline Alter \& intermaxilläre Raumlänge & 103 & $-0,285$ & 0,071 \\
\hline Alter \& Innenwinkel & 103 & 0,174 & 0,277 \\
\hline Alter \& Außenwinkel & 103 & $-0,132$ & 0,411 \\
\hline Alter \& LPW anterior-posterior & 73 & 0,028 & 0,860 \\
\hline Alter \& LPW transversal & 73 & $-0,084$ & 0,602 \\
\hline Alter \& LPW Fläche & 74 & 0,060 & 0,613 \\
\hline Alter \& Isthmus anterior-posterior & 100 & $-0,341$ & 0,029 \\
\hline Alter \& Isthmus transversal & 100 & 0,007 & 0,963 \\
\hline Alter \& Isthmus Fläche & 104 & $-0,031$ & 0,796 \\
\hline Alter \& Abstand Isthmus-Nasallinie & 104 & 0,229 & 0,150 \\
\hline & & &
\end{tabular}




\begin{tabular}{|l|l|l|l|} 
Alter \& TPW anterior-posterior & 104 & $-0,121$ & 0,452 \\
\hline Alter \& TPW transversal & 104 & 0,232 & 0,145 \\
\hline Alter \& Fläche TPW & 104 & 0,147 & 0,212 \\
\hline Alter \& Nasallinie-Unterkiefer & 104 & $-0,126$ & 0,433 \\
\hline Alter \& Abstand linguale Höcker & 104 & 0,086 & 0,594 \\
\hline Alter \& palatinale Höhe & 104 & 0,166 & 0,299 \\
\hline Alter \& Unterkieferlänge & 103 & 0,011 & 0,948 \\
\hline Alter \& BMI & 104 & $-0,009$ & 0,957 \\
\hline
\end{tabular}

Tab. 4: Korrelation Alter

Es besteht ein signifikanter Zusammenhang zwischen dem Alter der Patienten und der Länge des weichen Gaumens $(\mathrm{p}=0,003)$ und der Strecke Hyoid-Nasallinie ( $\mathrm{p}=0,008)$. Dabei gilt, je höher das Alter der Patienten ist, desto größer sind die entsprechenden Messwerte.

Weiterhin korreliert das Alter mit MPW anterior-posterior $(\mathrm{p}=0,002)$ und mit Isthmus anterior-posterior $(\mathrm{p}=0,029)$. Mit steigendem Alter nehmen die angegebenen Messwerte ab.

Die restlichen Messwerte ergeben keinen signifikanten Zusammenhang mit dem Alter.

\subsubsection{Body-Mass-Index (BMI)}

Der Zusammenhang zwischen dem BMI und den Messwerten wurde mithilfe von Korrelationsmatrizen nach Pearson ermittelt. Eine signifikante Korrelation wurde ab einem pWert $<0,05$ angenommen.

\begin{tabular}{|c|c|c|c|c|}
\hline \multirow{2}{*}{\multicolumn{2}{|c|}{$\begin{array}{l}\text { Korrelationsmatrizen nach Pearson } \\
\text { Variable }\end{array}$}} & \multicolumn{3}{|c|}{$\begin{array}{l}\text { markierte Korrelation } \\
\text { signifikant ab } p<0,050\end{array}$} \\
\hline & & \multirow{2}{*}{$\frac{N}{104}$} & \multirow{2}{*}{\begin{tabular}{|l|}
$\rho$ \\
0,190
\end{tabular}} & \multirow{2}{*}{\begin{tabular}{|l|} 
p-Niveau \\
0,185 \\
\end{tabular}} \\
\hline BMl & \& ANS-PNS & & & \\
\hline BMI & \& Länge weicher Gaumen & 104 & 0,100 & 0,488 \\
\hline BMI & \& Dicke weicher Gaumen & 58 & 0,059 & 0,686 \\
\hline BMI & \& PNS-Epiglottis & 103 & $-0,155$ & 0,281 \\
\hline BMI & \& Kontaktstrecke & 104 & 0,034 & 0,818 \\
\hline BMI & \& Hyoid-Nasallinie & 88 & 0,067 & 0,644 \\
\hline BMI & \& ANS-Hyoidsenkrechten & 88 & $-0,004$ & 0,970 \\
\hline BMI & \& Lufthöhe & 104 & 0,003 & 0,983 \\
\hline BMI & \& UPW anterior-posterior & 103 & $-0,157$ & 0,277 \\
\hline BMI & \& UPW transversal & 103 & $-0,434$ & $<0,001$ \\
\hline BMI & \& UPW Fläche & 103 & $-0,338$ & $<0,001$ \\
\hline BMI & \& MPW anterior-posterior & 102 & $-0,060$ & 0,554 \\
\hline BMI & \& MPW transversal & 102 & $-0,378$ & $<0,001$ \\
\hline BMI & \& Fläche MPW & 104 & $-0,204$ & 0,081 \\
\hline BMI & \& intermaxilläre Raumlänge & 103 & 0,208 & 0,039 \\
\hline BMI & \& Innenwinkel & 103 & $-0,135$ & 0,184 \\
\hline BMI & \& Außenwinkel & 103 & $-0,203$ & 0,040 \\
\hline BMI & \& LPW anterior-posterior & 73 & 0,131 & 0,271 \\
\hline BMI & \& LPW transversal & 73 & $-0,382$ & 0,001 \\
\hline BMI & \& LPW Fläche & 74 & $-0,210$ & 0,072 \\
\hline BMI & \& Isthmus anterior-posterior & 100 & $-0,107$ & 0,370 \\
\hline BMI & \& Isthmus transversal & 100 & $-0,453$ & $<0,001$ \\
\hline
\end{tabular}




\begin{tabular}{|ll|l|l|l|} 
BMI \& Isthmus Fläche & 104 & $-0,305$ & 0,008 \\
\hline BMI \& Abstand Isthmus-Nasallinie & 104 & 0,046 & 0,701 \\
\hline BMI \& TPW anterior-posterior & 104 & 0,188 & 0,155 \\
\hline BMI \& TPW transversal & 104 & $-0,253$ & 0,032 \\
\hline BMI \& Fläche TPW & 104 & $-0,067$ & 0,573 \\
\hline BMI \& Nasallinie-Unterkiefer & 104 & 0,195 & 0,048 \\
\hline BMI \& Abstand linguale Höcker & 104 & 0,249 & 0,011 \\
\hline BMI \& palatinale Höhe & 104 & 0,172 & 0,082 \\
\hline BMI \& Unterkieferlänge & 103 & 0,118 & 0,234 \\
\hline BMI \& Alter & 104 & 0,028 & 0,776 \\
\hline
\end{tabular}

Tab. 5: Korrelation BMI

Es besteht ein signifikanter Zusammenhang zwischen dem BMI der Patienten und den Strecken UPW transversal $(\mathrm{p}<0,001)$, MPW transversal $(\mathrm{p}<0,001)$, Isthmus transversal ( $p<0,001)$, TPW transversal $(p=0,032)$, LPW transversal $(p=0,001)$, sowie den Flächen auf Höhe UPW ( $\mathrm{p}<0,001)$, LPW ( $\mathrm{p}=0,072)$ und Isthmus $(\mathrm{p}=0,008)$. Dabei gilt je größer der BMI der Patienten, desto kleiner sind die entsprechenden Messwerte. Weiterhin korreliert der BMI mit der intermaxillären Raumlänge $(p=0,039)$, dem Außenwinkel $(p=0,040)$, der Strecke Nasallinie - Unterkiefer $(\mathrm{p}=0,048)$, sowie mit dem Abstand der lingualen Höcker $(\mathrm{p}=0,011)$. Mit steigendem BMI-Wert steigen auch die Messwerte an. Die restlichen Messwerte haben keinen signifikanten Zusammenhang mit BMI.

\subsubsection{Epworth Sleepiness Scale (ESS)}

Der Zusammenhang zwischen den Messwerten und den ESS-Werten wurde mit der Spearman Rangkorrelation ermittelt.

Eine signifikante Korrelation wurde ab einem p-Wert $<0,05$ angenommen.

\begin{tabular}{|l|l|l|l|}
\hline Spearmans Rangkorrelation & & & \\
\hline Variable & $\mathrm{N}$ & Spearman R & p-Niveau \\
\hline ESS \& ANS-PNS & 104 & 0,116 & 0,243 \\
\hline ESS \& Länge weicher Gaumen & 104 & $-0,045$ & 0,650 \\
\hline ESS \& Dicke weicher Gaumen & 58 & 0,158 & 0,238 \\
\hline ESS \& PNS-Epiglottis & 103 & $-0,017$ & 0,862 \\
\hline ESS \& Kontaktstrecke & 104 & 0,008 & 0,934 \\
\hline ESS \& Hyoid-Nasallinie & 88 & $-0,247$ & 0,020 \\
\hline ESS \& ANS-Hyoidsenkrechten & 88 & 0,130 & 0,227 \\
\hline ESS \& Lufthöhe & 104 & 0,096 & 0,331 \\
\hline ESS \& UPW anterior-posterior & 103 & $-0,156$ & 0,117 \\
\hline ESS \& UPW transversal & 103 & $-0,035$ & 0,725 \\
\hline ESS \& UPW Fläche & 103 & $-0,122$ & 0,216 \\
\hline ESS \& MPW anterior-posterior & 102 & $-0,010$ & 0,924 \\
\hline ESS \& MPW transversal & 102 & 0,015 & 0,880 \\
\hline ESS \& MPW Fläche & 104 & $-0,001$ & 0,993 \\
\hline
\end{tabular}




\begin{tabular}{|l|l|l|l|l|} 
ESS \& intermaxilläre Raumlänge & 103 & $-0,110$ & 0,269 \\
\hline ESS \& Innenwinkel & 103 & $-0,109$ & 0,271 \\
\hline ESS \& Außenwinkel & 103 & $-0,192$ & 0,052 \\
\hline ESS \& LPW anterior-posterior & 73 & $-0,176$ & 0,136 \\
\hline ESS \& LPW transversal & 73 & 0,081 & 0,498 \\
\hline ESS \& LPW Fläche & 74 & $-0,090$ & 0,447 \\
\hline ESS \& Isthmus anterior-posterior & 100 & $-0,217$ & 0,030 \\
\hline ESS \& Isthmus transversal & 100 & $-0,061$ & 0,545 \\
\hline ESS \& Isthmus Fläche & 104 & $-0,121$ & 0,222 \\
\hline ESS \& Abstand Isthmus-Nasallinie & 104 & $-0,098$ & 0,322 \\
\hline ESS \& TPW anterior-posterior & 104 & $-0,142$ & 0,151 \\
\hline ESS \& TPW transversal & 104 & $-0,168$ & 0,088 \\
\hline ESS \& Fläche TPW & 104 & $-0,180$ & 0,067 \\
\hline ESS \& Nasallinie-Unterkiefer & 104 & $-0,161$ & 0,102 \\
\hline ESS \& Abstand linguale Höcker & 104 & $-0,090$ & 0,363 \\
\hline ESS \& palatinale Höhe & 104 & $-0,139$ & 0,158 \\
\hline ESS \& Unterkieferlänge & 103 & $-0,056$ & 0,574 \\
\hline ESS \& Alter & 104 & $-0,008$ & 0,935 \\
\hline ESS \& BMI & 104 & $-0,011$ & 0,914 \\
\hline
\end{tabular}

Tab. 6: Korrelation ESS

Der Test zeigt einen signifikanten Zusammenhang zwischen dem ESS-Wert und der Strecke Hyoid-Nasalline $(\mathrm{p}=0,020)$, wobei eine verringerte Strecke Hyoid-Nasallinie einen erhöhten ESS-Wert aufweist.

Des Weiteren ergibt sich ein signifikanter Zusammenhang zwischen dem ESS-Wert und der Strecke Isthmus anterior-posterior $(\mathrm{p}=0,030)$, wobei der ESS-Wert mit Verkleinerung der anterior-posterioren Isthmusstrecke zunimmt. Wie der Test zeigt, ergeben die anderen Messwerte keinen signifikanten Zusammenhang mit der Höhe des ESS-Wertes.

\subsubsection{Lautstärke des Schnarchens}

Ein möglicher Zusammenhang zwischen dem Wert für die Lautstärke und den Messwerten wurde mithilfe der Spearman Rangkorrelation ermittelt.

Eine signifikante Korrelation wurde ab einem p-Wert $<0,05$ angenommen.

\begin{tabular}{|l|l|l|l|}
\hline Spearmans Rangkorrelation & & & \\
\hline Variable & $\mathrm{N}$ & Spearman R & p-Niveau \\
\hline Lautstärke \& ANS-PNS & 101 & 0,200 & 0,045 \\
\hline Lautstärke \& Länge weicher Gaumen & 101 & 0,408 & $<0,001$ \\
\hline Lautstärke \& Dicke weicher Gaumen & 56 & 0,142 & 0,297 \\
\hline Lautstärke \& PNS-Epiglottis & 100 & 0,339 & 0,001 \\
\hline Lautstärke \& Kontaktstrecke & 101 & 0,013 & 0,897 \\
\hline Lautstärke \& Hyoid-Nasallinie & 85 & 0,231 & 0,034 \\
\hline Lautstärke \& ANS-Hyoidsenkrechte & 85 & 0,148 & 0,178 \\
\hline Lautstärke \& Lufthöhe & 101 & 0,068 & 0,499 \\
\hline Lautstärke \& UPW anterior-posterior & 100 & $-0,083$ & 0,411 \\
\hline
\end{tabular}




\begin{tabular}{|l|l|l|l|}
\hline Lautstärke \& UPW transversal & 100 & $-0,106$ & 0,293 \\
\hline Lautstärke \& UPW Fläche & 100 & $-0,076$ & 0,451 \\
\hline Lautstärke \& MPW anterior-posterior & 100 & $-0,276$ & 0,005 \\
\hline Lautstärke \& MPW transversal & 100 & $-0,167$ & 0,097 \\
\hline Lautstärke \& MPW Fläche & 101 & $-0,301$ & 0,002 \\
\hline Lautstärke \& intermaxilläre Raumlänge & 100 & 0,005 & 0,963 \\
\hline Lautstärke \& Innenwinkel & 100 & $-0,054$ & 0,595 \\
\hline Lautstärke \& Außenwinkel & 100 & $-0,346$ & $<0,001$ \\
\hline Lautstärke \& LPW anterior-posterior & 71 & $-0,070$ & 0,562 \\
\hline Lautstärke \& LPW transversal & 71 & $-0,020$ & 0,866 \\
\hline Lautstärke \& LPW Fläche & 72 & $-0,037$ & 0,758 \\
\hline Lautstärke \& Isthmus anterior-posterior & 98 & $-0,326$ & 0,001 \\
\hline Lautstärke \& Isthmus transversal & 98 & $-0,159$ & 0,117 \\
\hline Lautstärke \& Isthmus Fläche & 101 & $-0,280$ & 0,005 \\
\hline Lautstärke \& Abstand Isthmus-Nasallinie & 101 & 0,274 & 0,006 \\
\hline Lautstärke \& TPW anterior-posterior & 101 & $-0,020$ & 0,841 \\
\hline Lautstärke \& TPW transversal & 101 & $-0,018$ & 0,858 \\
\hline Lautstärke \& Fläche TPW & 101 & $-0,045$ & 0,652 \\
\hline Lautstärke \& Nasallinie-Unterkiefer & 101 & 0,048 & 0,634 \\
\hline Lautstärke \& Abstand linguale Höcker & 101 & $-0,006$ & 0,954 \\
\hline Lautstärke \& palatinale Höhe & 101 & 0,057 & 0,574 \\
\hline Lautstärke \& Unterkiefer-Länge & 100 & 0,109 & 0,281 \\
\hline Lautstärke \& Alter & 101 & 0,222 & 0,025 \\
\hline Lautstärke \& BMI & 101 & 0,401 & $<0,001$ \\
\hline Tab 7: Korelon Laust & &
\end{tabular}

Tab. 7: Korrelation Lautstärke

Der Test zeigt einen signifikanten Zusammenhang zwischen der Lautstärke des Schnarchens und der Strecke ANS-PNS ( $\mathrm{p}=0,045)$, wobei ein verlängerter harter Gaumen zu einer erhöhten Lautstärke führt.

Ein signifikanter Zusammenhang ergibt sich zwischen der Lautstärke und der Länge des weichen Gaumens $(p<0,001)$. Dabei nimmt die Lautstärke mit verlängertem weichen Gaumen $\mathrm{zu}$.

Es besteht ein signifikanter Zusammenhang zwischen der Lautstärke und der Strecke PNSEpiglottis $(p=0,001)$, der Strecke Hyoid-Nasallinie $(p=0,034)$, dem Abstand IsthmusNasallinie $(p=0,006)$, dem Alter $(p=0,025)$ und dem BMI $(p<0,001)$. Mit zunehmender Größe der Messwerte steigt auch die Lautstärke des Schnarchens an.

Des Weiteren zeigt sich ein signifikanter Zusammenhang zwischen der Lautstärke des Schnarchens und MPW anterior-posterior $(\mathrm{p}=0,005)$, Fläche MPW ( $\mathrm{p}=0,002)$, Außenwinkel ( $p<0,001)$, Isthmus anterior-posterior $(p=0,001)$ und Fläche Isthmus $(p=0,005)$. Bei diesen Messparametern steigt die Lautstärke mit abnehmender Größe der Messwerte an. 


\subsubsection{Häufigkeit des Schnarchens}

Ein möglicher Zusammenhang zwischen dem Wert für die Häufigkeit des Schnarchens und den Messwerten wurde mithilfe der Spearman Rangkorrelation ermittelt.

Eine signifikante Korrelation wurde ab einem p-Wert $<0,05$ angenommen.

\begin{tabular}{|l|l|l|l|}
\hline Spearmans Rangkorrelation & & & \\
\hline Variable & N & Spearman R & p-Niveau \\
\hline Häufigkeit \& ANS-PNS & 98 & 0,116 & 0,254 \\
\hline Häufigkeit \& Länge weicher Gaumen & 98 & 0,364 & $<0,001$ \\
\hline Häufigkeit \& Dicke weicher Gaumen & 55 & 0,195 & 0,154 \\
\hline Häufigkeit \& PNS-Epiglottis & 97 & 0,249 & 0,014 \\
\hline Häufigkeit \& Kontaktstrecke & 98 & $-0,054$ & 0,595 \\
\hline Häufigkeit \& Hyoid-Nasallinie & 82 & 0,218 & 0,049 \\
\hline Häufigkeit \& ANS-Hyoidsenkrechte & 82 & 0,116 & 0,301 \\
\hline Häufigkeit \& Lufthöhe & 98 & 0,112 & 0,274 \\
\hline Häufigkeit \& UPW anterior-posterior & 97 & $-0,028$ & 0,788 \\
\hline Häufigkeit \& UPW transversal & 97 & $-0,081$ & 0,433 \\
\hline Häufigkeit \& UPW Fläche & 97 & $-0,026$ & 0,800 \\
\hline Häufigkeit \& MPW anterior-posterior & 97 & $-0,207$ & 0,042 \\
\hline Häufigkeit \& MPW transversal & 97 & $-0,188$ & 0,065 \\
\hline Häufigkeit \& MPW Fläche & 98 & $-0,256$ & 0,011 \\
\hline Häufigkeit \& intermaxilläre Raumlänge & 97 & $-0,059$ & 0,569 \\
\hline Häufigkeit \& Innenwinkel & 97 & $-0,063$ & 0,537 \\
\hline Häufigkeit \& Außenwinkel & 97 & $-0,377$ & $<0,001$ \\
\hline Häufigkeit \& LPW anterior-posterior & 70 & 0,017 & 0,887 \\
\hline Häufigkeit \& LPW transversal & 70 & $-0,005$ & 0,965 \\
\hline Häufigkeit \& LPW Fläche & 71 & $-0,003$ & 0,981 \\
\hline Häufigkeit \& Isthmus anterior-posterior & 95 & $-0,348$ & 0,001 \\
\hline Häufigkeit \& Isthmus transversal & 95 & $-0,283$ & 0,005 \\
\hline Häufigkeit \& Isthmus Fläche & 98 & $-0,362$ & $<0,001$ \\
\hline Häufigkeit \& Abstand Isthmus-Nasallinie & 98 & 0,190 & 0,061 \\
\hline Häufigkeit \& TPW anterior-posterior & 98 & 0,065 & 0,526 \\
\hline Häufigkeit \& TPW transversal & 98 & 0,039 & 0,699 \\
\hline Häufigkeit \& Fläche TPW & 98 & 0,052 & 0,610 \\
\hline Häufigkeit \& Nasallinie-Unterkiefer & 98 & 0,015 & 0,882 \\
\hline Häufigkeit \& Abstand linguale Höcker & 98 & $-0,099$ & 0,990 \\
\hline Häufigkeit \& palatale Höhe & 98 & 0,001 & 0,472 \\
\hline Häufigkeit \& Unterkieferlänge & 97 & 0,074 & $<0,001$ \\
\hline Häufigkeit \& Alter & 98 & 0,372 & $<0,001$ \\
\hline Häufigkeit \& BMI & 98 & 0,367 & \\
\hline & & & \\
\hline
\end{tabular}

Tab. 8: Korrelation Häufigkeit

Es besteht ein signifikanter Zusammenhang zwischen der Häufigkeit des Schnarchens und der Länge des weichen Gaumens ( $p<0,001)$, der Strecke PNS-Epiglottis $(p=0,014)$, der Strecke Hyoid-Nasallinie $(\mathrm{p}=0,049)$, dem Alter $(\mathrm{p}<0,001)$ und dem BMI $(\mathrm{p}<0,001)$. Mit zunehmender Größe der Messwerte steigt auch die Häufigkeit des Schnarchens an. 
Des Weiteren zeigt sich ein signifikanter Zusammenhang zwischen der Häufigkeit des Schnarchens und MPW anterior-posterior $(\mathrm{p}=0,042)$, Fläche MPW ( $\mathrm{p}=0,011)$, Außenwinkel ( $p<0,001)$, Isthmus anterior-posterior $(p=0,001)$, Isthmus transversal $(p=0,005)$ und Fläche Isthmus $(\mathrm{p}<0,001)$. Bei diesen Messparametern steigt die Häufigkeit des Schnarchens mit abnehmender Größe der Messwerte.

Wie der Test zeigt, ergeben die anderen Messwerte keinen signifikanten Zusammenhang mit der Häufigkeit des Schnarchens.

\subsection{Betrachtung des orofazialen Systemzustandes in Relation zu radiologischen Messwerten}

Mithilfe des Mann-Whitney-U-Test wurden die gemessenen Werte und Daten aus dem Fragebogen (BMI, ESS-Wert, Lautstärke und Häufigkeit des Schnarchens) mit dem Funktionszustand des orofazialen Systems verglichen. Ein signifikanter Unterschied zwischen den Systemzuständen wurde ab einem p-Wert <0,05 angenommen.

\begin{tabular}{|l|l|} 
& \\
& p-Niveau \\
\hline ANS-PNS & 0,086 \\
\hline Länge weicher Gaumen & 0,505 \\
\hline Dicke weicher Gaumen & 1,000 \\
\hline PNS-Epiglottis & 0,717 \\
\hline Kontaktstrecke & $<0,001$ \\
\hline Hyoid-Nasallinie & 0,133 \\
\hline ANS-Hyoidsenkrechten & 0,203 \\
\hline Lufthöhe & $<0,001$ \\
\hline UPW anterior-posterior & 0,595 \\
\hline UPW transversal & 0,192 \\
\hline UPW Fläche & 0,177 \\
\hline MPW anterior-posterior & 0,467 \\
\hline MPW transversal & 0,019 \\
\hline MPW Fläche & 0,072 \\
\hline Intermaxilläre Raumlänge & 0,867 \\
\hline Innenwinkel & 0,088 \\
\hline Außenwinkel & 0,857 \\
\hline LPW anterior-posterior & 0,481 \\
\hline LPW transversal & 0,124 \\
\hline LPW Fläche & 0,629 \\
\hline Isthmus anterior-posterior & 0,247 \\
\hline Isthmus transversal & 0,035 \\
\hline Isthmus Fläche & 0,016 \\
\hline Abstand Isthmus-Nasallinie & 0,173 \\
\hline TPW anterior-posterior & 0,827 \\
\hline & \\
\hline
\end{tabular}




\begin{tabular}{|l|l|} 
TPW transversal & 0,078 \\
\hline Fläche TPW & 0,292 \\
\hline Nasallinie-Unterkiefer & 0,758 \\
\hline Abstand linguale Höcker & 0,111 \\
\hline palatinale Höhe & 0,613 \\
\hline Unterkieferlänge & 0,925 \\
\hline Alter & 0,535 \\
\hline BMI & 0,076 \\
\hline ESS & 0,641 \\
\hline Lautstärke & 0,338 \\
\hline Häufigkeit & 0,685 \\
\hline
\end{tabular}

Tab. 9: Einfluss Systemzustand

Die Tabelle 9 zeigt einen signifikanten Unterschied zwischen dem Systemzustand offen und geschlossen bezüglich der Kontaktstrecke zwischen dem weichen Gaumen und der Zunge $(\mathrm{p}<0,001)$, der Lufthöhe unter dem harten Gaumen $(\mathrm{p}<0,001)$, MPW transversal $(\mathrm{p}=0,019)$, Isthmus transversal ( $\mathrm{p}=0,035)$ und der Fläche auf Höhe Isthmus $(\mathrm{p}=0,016)$.

Zur Veranschaulichung der gefundenen Unterschiede bezüglich des Systemzustandes wurden Box-Plots verwendet.

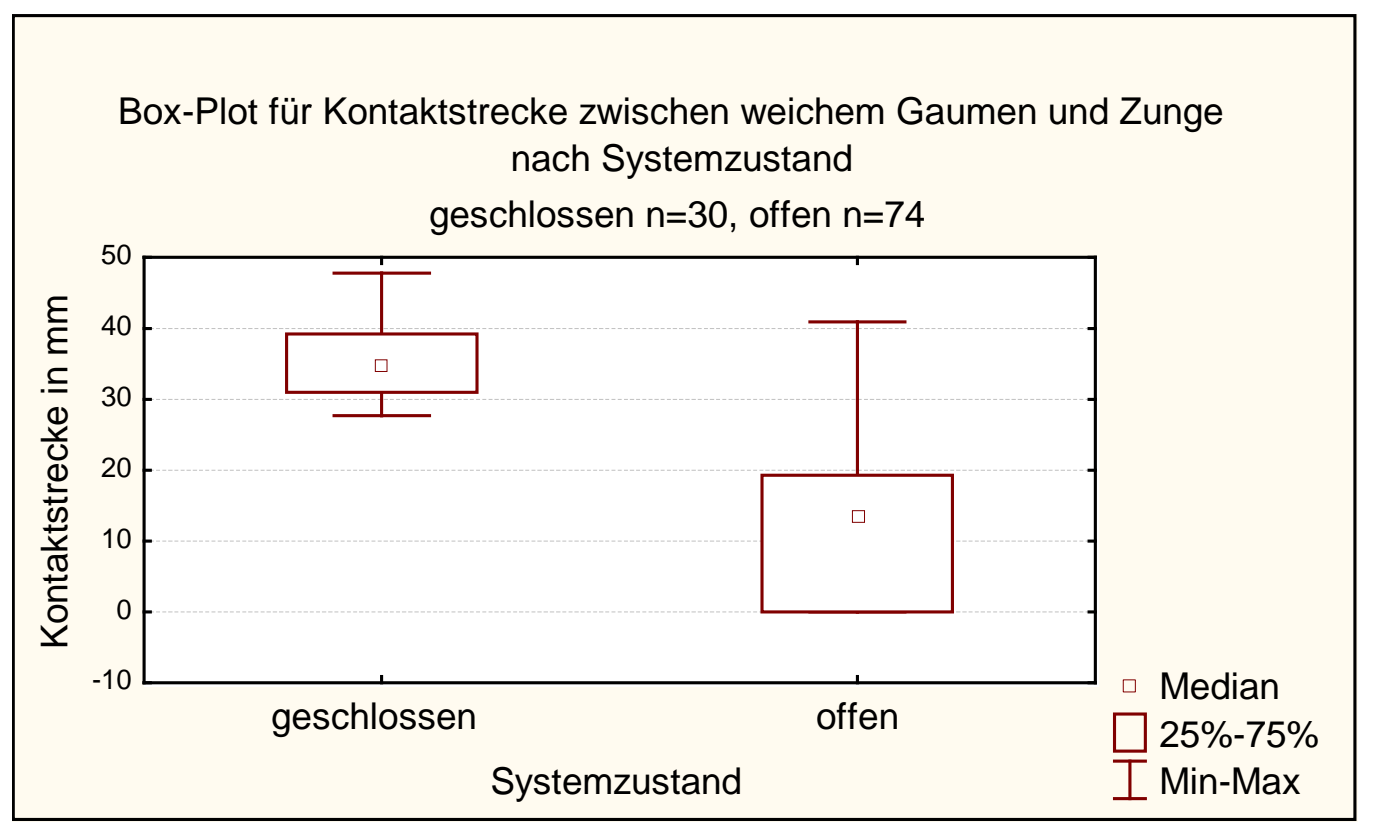

Abb. 14: Box-Plot für Kontaktstrecke und Systemzustand 


\begin{tabular}{|l|c|c|}
\hline $\begin{array}{l}\text { Kontaktstrecke } \\
(\mathrm{mm})\end{array}$ & $\begin{array}{l}\text { System geschlossen } \\
\mathrm{N}=30\end{array}$ & $\begin{array}{l}\text { System } \\
\text { offen } \\
\mathrm{N}=74\end{array}$ \\
\hline Median & 34,7 & 13,6 \\
\hline Maximum & 47,8 & 40,9 \\
\hline Minimum & 27,7 & 0,0 \\
\hline
\end{tabular}

Tab. 10: Kontaktstrecke und Systemzustand

Die Abbildung 14 und Tabelle 10 zeigen einen Unterschied zwischen den Probanden mit einem offenen und einem geschlossenen Systemzustand in Bezug auf die Kontaktstrecke zwischen dem weichen Gaumen und der Zunge. Probanden mit einem geschlossenen Systemzustand besitzen tendenziell eine größere Kontaktstrecke zwischen dem weichen Gaumen und der Zunge.

Box-Plot für die Lufthöhe unter dem harten Gaumen nach Systemzustand geschlossen $n=30$, offen $n=74$

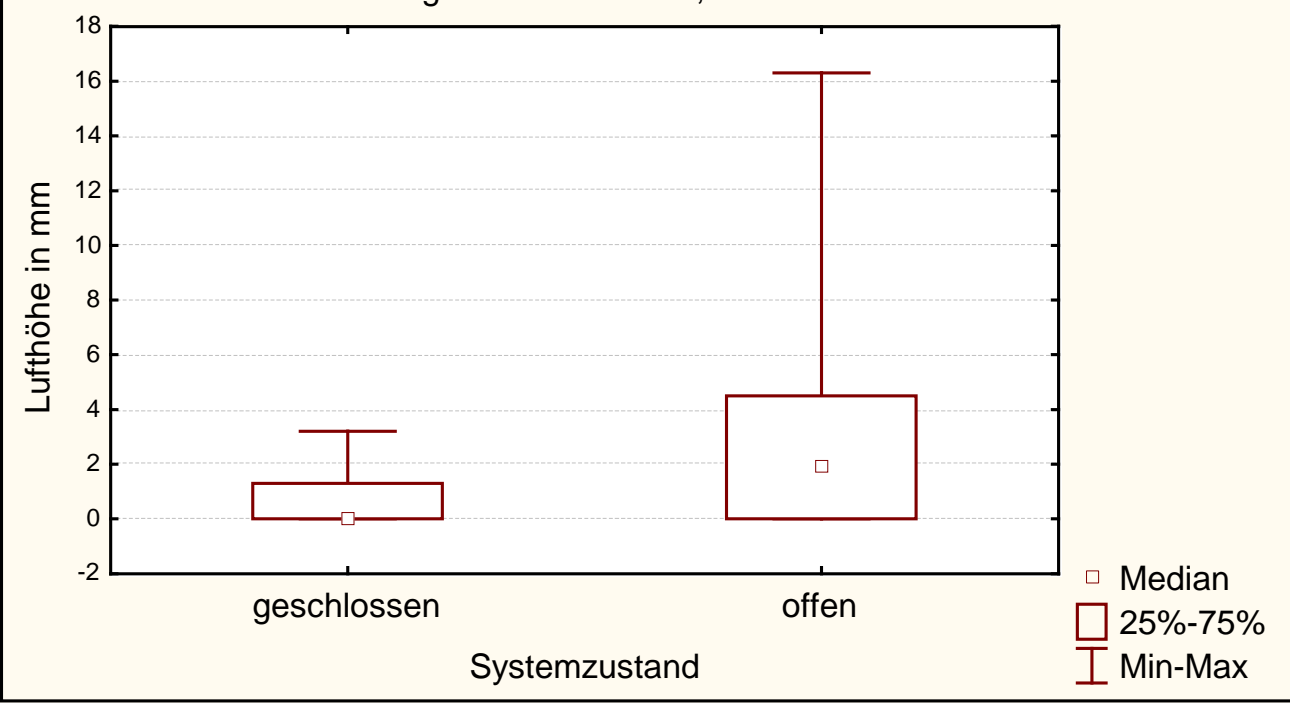

Abb. 15: Box-Plot für Lufthöhe und Systemzustand

\begin{tabular}{|l|l|l|}
\hline $\begin{array}{l}\text { Lufthöhe } \\
\text { (mm) }\end{array}$ & $\begin{array}{l}\text { System } \\
\text { geschlossen } \\
\mathrm{N}=30\end{array}$ & $\begin{array}{l}\text { System } \\
\text { offen } \\
\mathrm{N}=74\end{array}$ \\
\hline Median & 0,0 & 2,0 \\
\hline Maximum & 3,2 & 16,3 \\
\hline Minimum & 0 & 0 \\
\hline
\end{tabular}

Tab. 11: Lufthöhe und Systemzustand

Die Abbildung 15 und Tabelle 11 zeigen einen geringen Unterschied zwischen den Probanden mit einem offenen und einem geschlossenen Systemzustand bezüglich der Lufthöhe unter dem harten Gaumen. Probanden mit einem offenen Systemzustand besitzen tendenziell eine größere Lufthöhe unter dem harten Gaumen. 


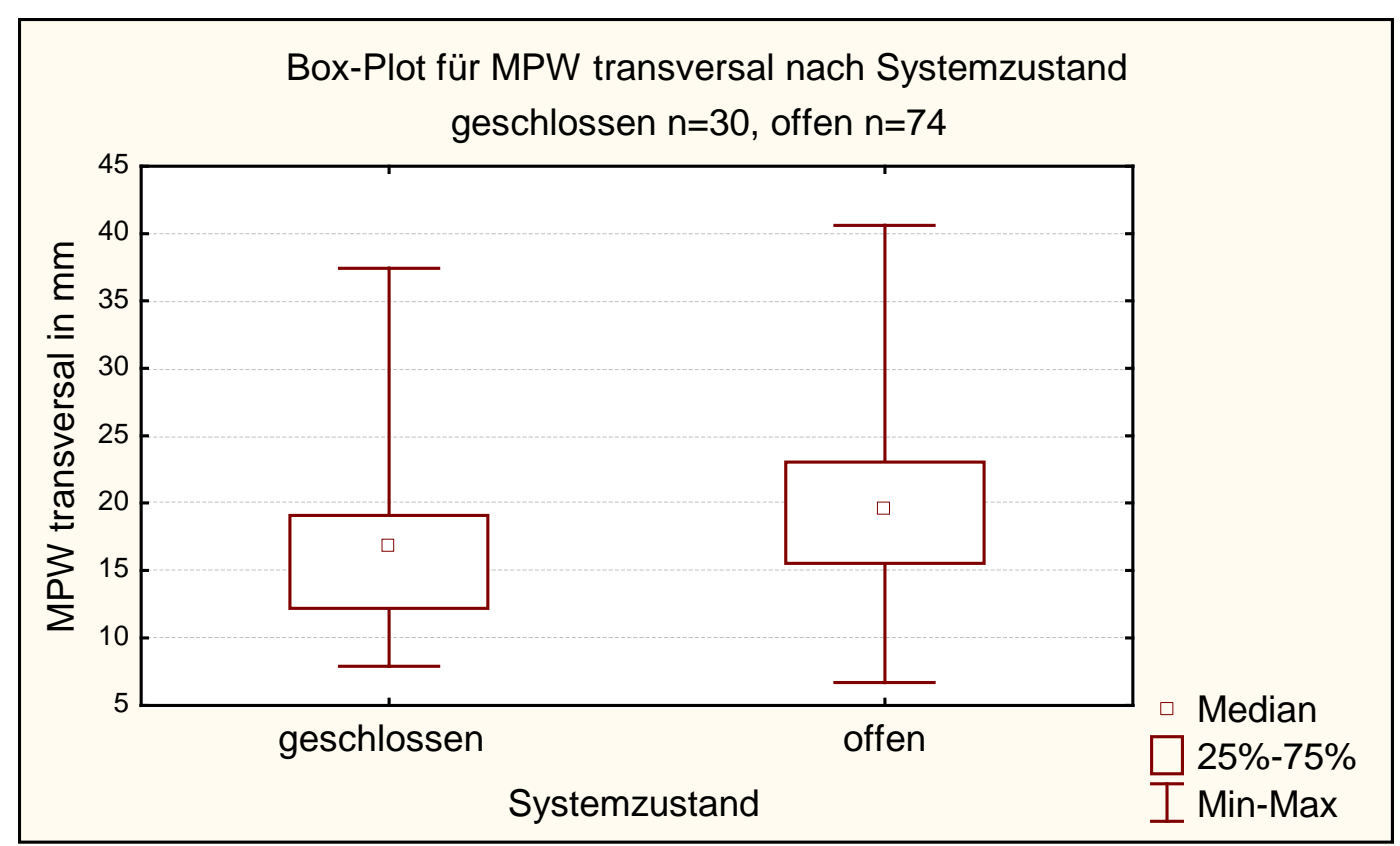

Abb. 16: Box-Plot für MPW transversal und Systemzustand

\begin{tabular}{|l|l|l|}
\hline $\begin{array}{l}\text { MPW } \\
\text { transversal } \\
(\mathrm{mm})\end{array}$ & $\begin{array}{l}\text { System } \\
\text { geschlossen } \\
\mathrm{N}=30\end{array}$ & $\begin{array}{l}\text { System } \\
\text { offen } \\
\mathrm{N}=74\end{array}$ \\
\hline Median & 17,0 & 19,6 \\
\hline Maximum & 37,4 & 40,6 \\
\hline Minimum & 7,9 & 6,7 \\
\hline
\end{tabular}

Tab. 12: Fläche MPW transversal und Systemzustand

In Bezug auf die transversale Ausdehnung des Luftweges auf Höhe MPW zeigen die Abbildung 16 und Tabelle 12 einen geringen Unterschied zwischen den Probanden mit einem offenen und einem geschlossenen Systemzustand. Probanden mit einem offenen Systemzustand besitzen tendenziell eine größere transversale Ausdehnung des Luftweges auf Höhe MPW . 


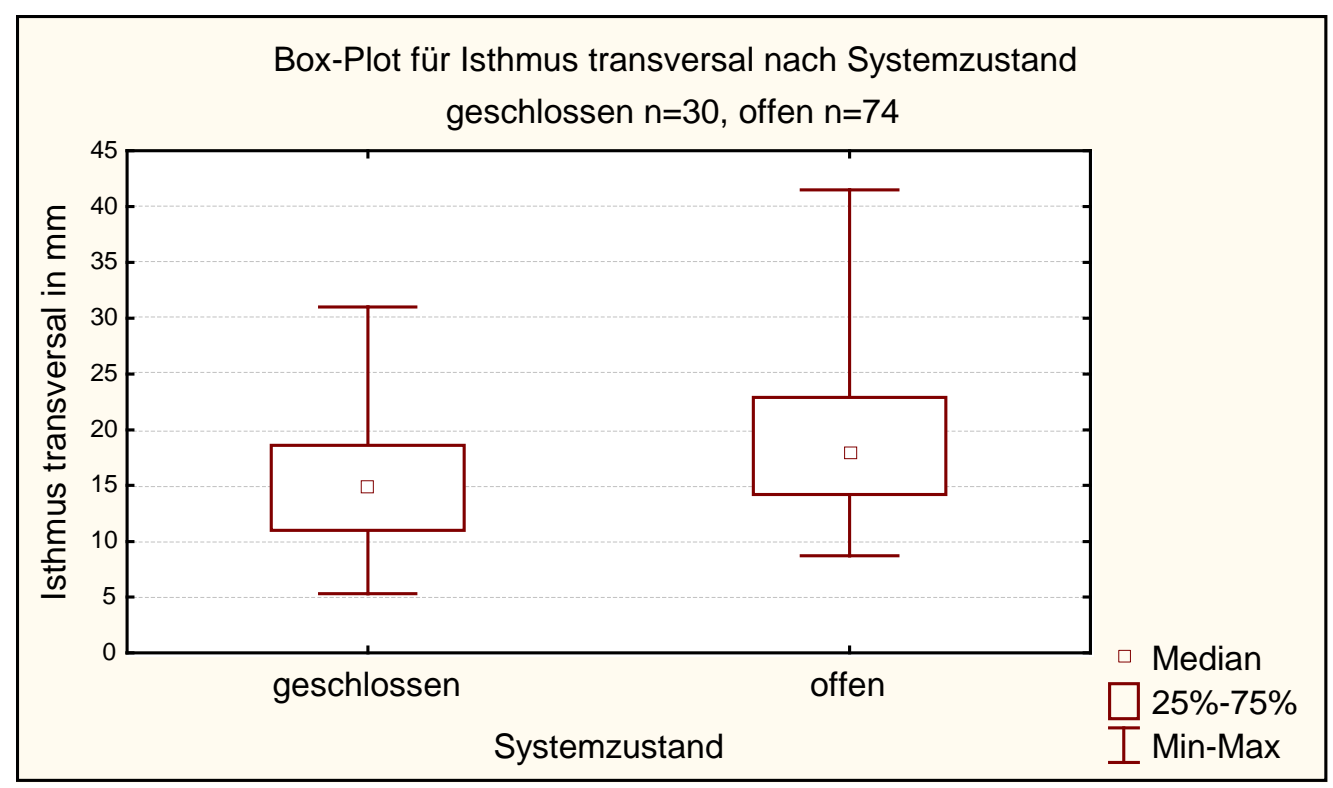

Abb. 17: Box-Plot für Isthmus transversal und Systemzustand

\begin{tabular}{|l|l|l|}
\hline $\begin{array}{l}\text { Isthmus } \\
\text { transversal } \\
(\mathrm{mm})\end{array}$ & $\begin{array}{l}\text { System } \\
\text { geschlossen } \\
\mathrm{N}=30\end{array}$ & $\begin{array}{l}\text { System } \\
\text { offen } \\
\mathrm{N}=74\end{array}$ \\
\hline Median & 15,0 & 18,0 \\
\hline Maximum & 31,0 & 41,5 \\
\hline Minimum & 5,3 & 8,7 \\
\hline
\end{tabular}

Tab. 13: Fläche Isthmus transversal und Systemzustand

Die Abbildung 17 und Tabelle 13 zeigen einen geringen Unterschied zwischen den Probanden mit einem offenen und einem geschlossenen Systemzustand bezüglich der transversalen Ausdehnung des Luftweges auf Höhe Isthmus. Probanden mit einem offenen Systemzustand besitzen auf Höhe Isthmus tendenziell eine größere transversale Ausdehnung des Luftweges. 


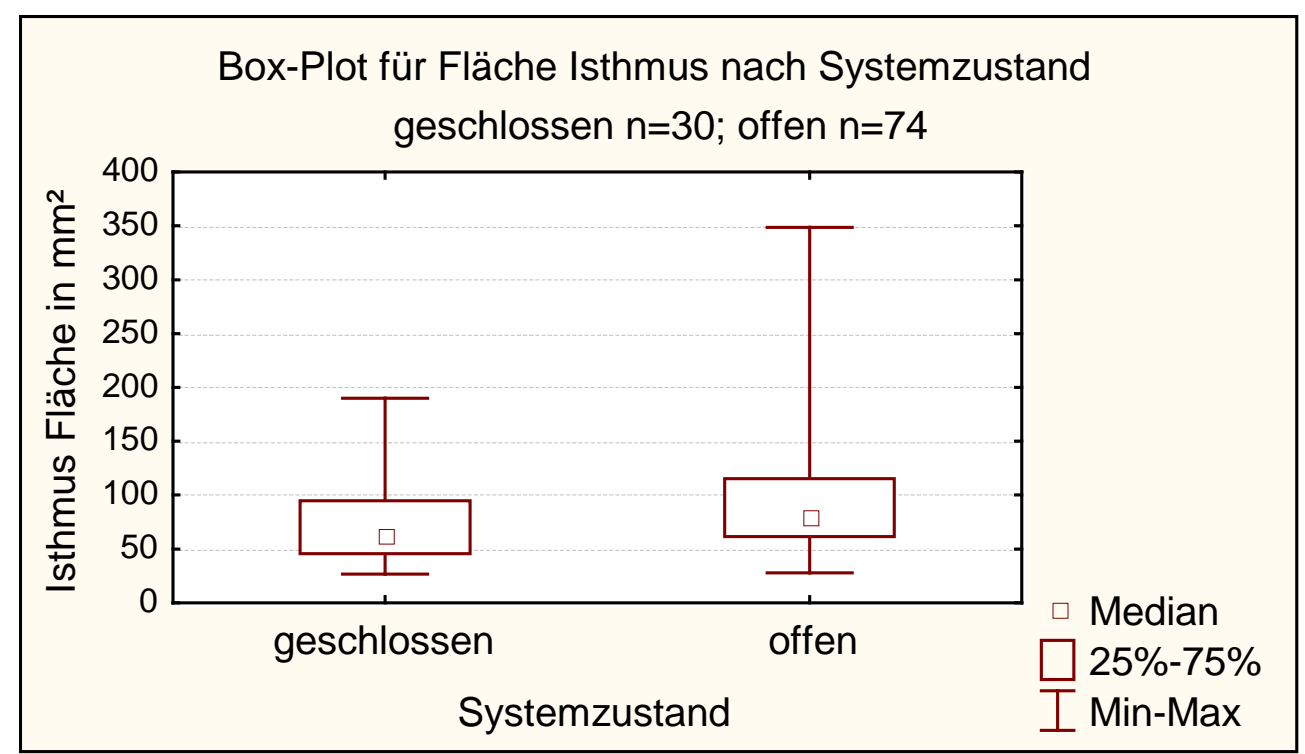

Abb. 18: Box-Plot für Fläche Isthmus und Systemzustand

\begin{tabular}{|l|l|l|}
\hline $\begin{array}{l}\text { Isthmus } \\
\text { Fläche } \\
\left(\mathrm{mm}^{2}\right)\end{array}$ & $\begin{array}{l}\text { System } \\
\text { geschlossen } \\
\mathrm{N}=30\end{array}$ & $\begin{array}{l}\text { System } \\
\text { offen } \\
\mathrm{N}=74\end{array}$ \\
\hline Median & 64,35 & 80,68 \\
\hline Maximum & 189,90 & 348,75 \\
\hline Minimum & 26,64 & 28,01 \\
\hline
\end{tabular}

Tab. 14: Fläche Isthmus und Systemzustand

In Bezug auf die Fläche des Luftweges auf Höhe Isthmus zeigen die Abbildung 18 und Tabelle 14 einen geringen Unterschied zwischen den Probanden mit einem offenen und einem geschlossenen Systemzustand. Probanden mit einem offenen Systemzustand besitzen tendenziell eine größere Fläche des Luftweges auf Höhe Isthmus. 


\subsection{Fallbeispiele}

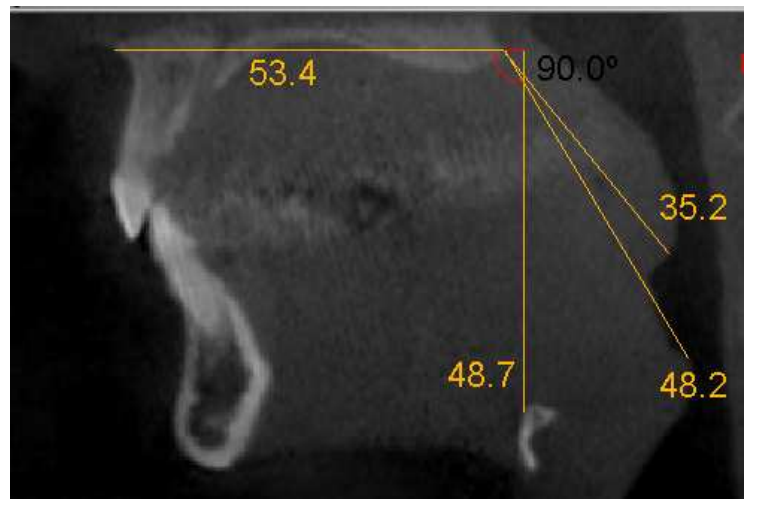

Abb. 19: sagittale Vermessung (1)

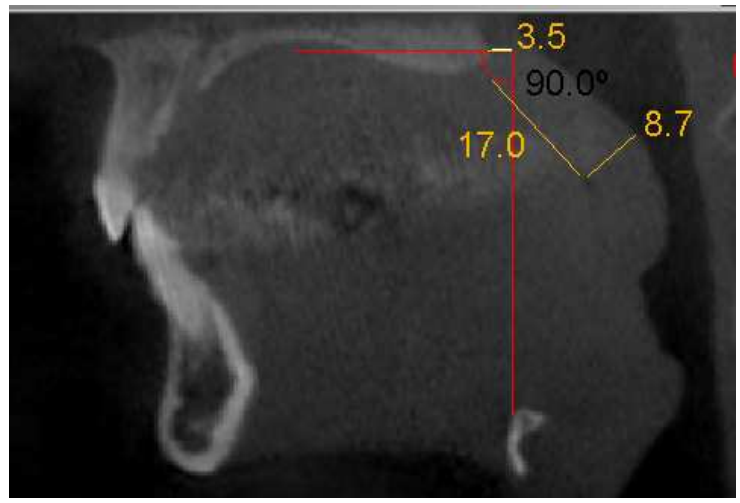

Abb. 20: sagittale Vermessung (2)

Die Abbildungen 19 und 20 zeigen die Vermessung mediosagittaler Schnitte.

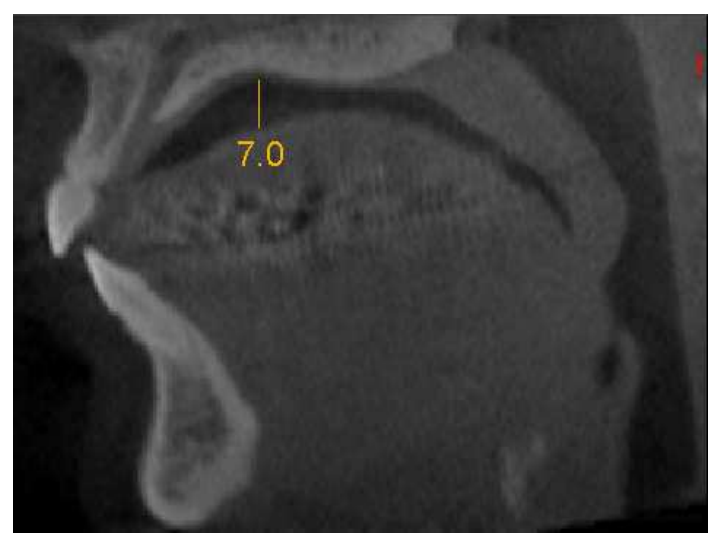

Abb. 21: sagittale Vermessung (3)

Die Abbildung 21 zeigt die Vermessung einer vorhandenen Lufthöhe unter dem harten Gaumen, des Weiteren zeigt sich ein offener Systemzustand. 


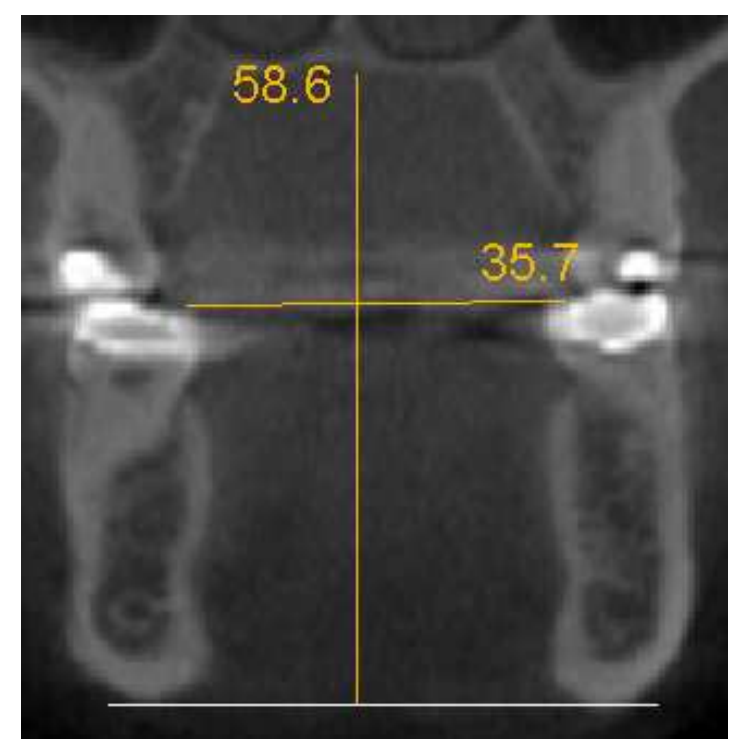

Abb. 22: Vermessung koronaler Schnitt (1)

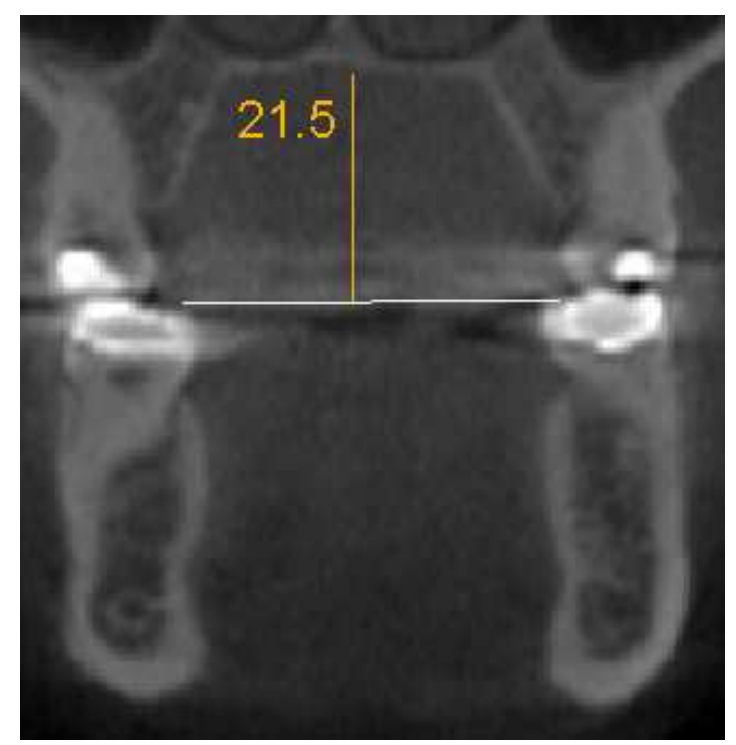

Abb. 23: Vermessung koronaler Schnitt (2)

Die Abbildungen 22 und 23 zeigen die Vermessung des koronalen Schnittes.

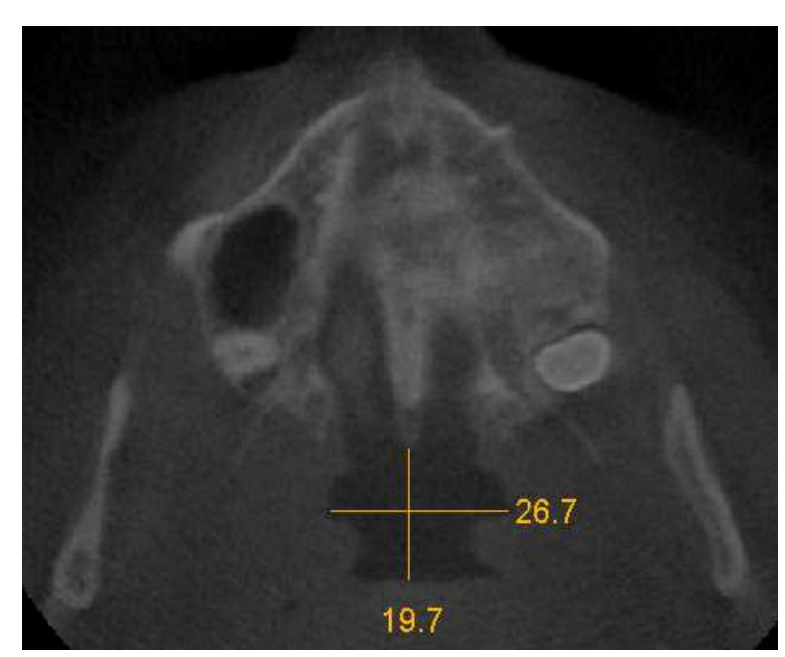

Abb. 24: axiale Vermessung auf Höhe UPW

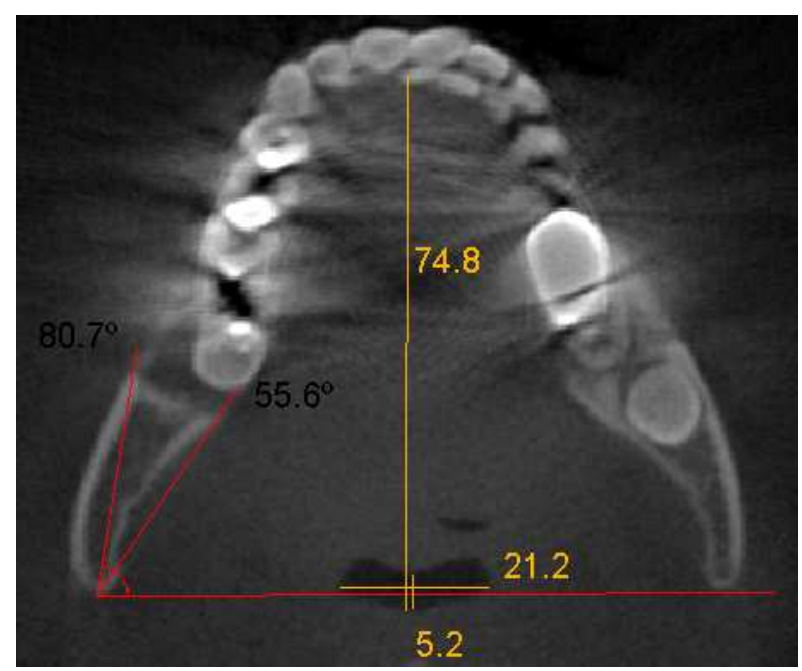

Abb. 26: axiale Vermessung auf Höhe MPW

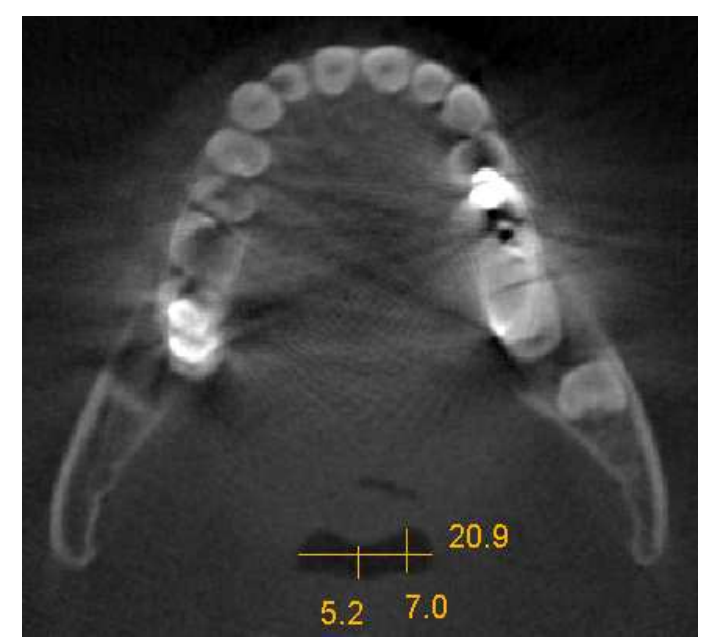

Abb. 25: axiale Vermessung auf Höhe Isthmus

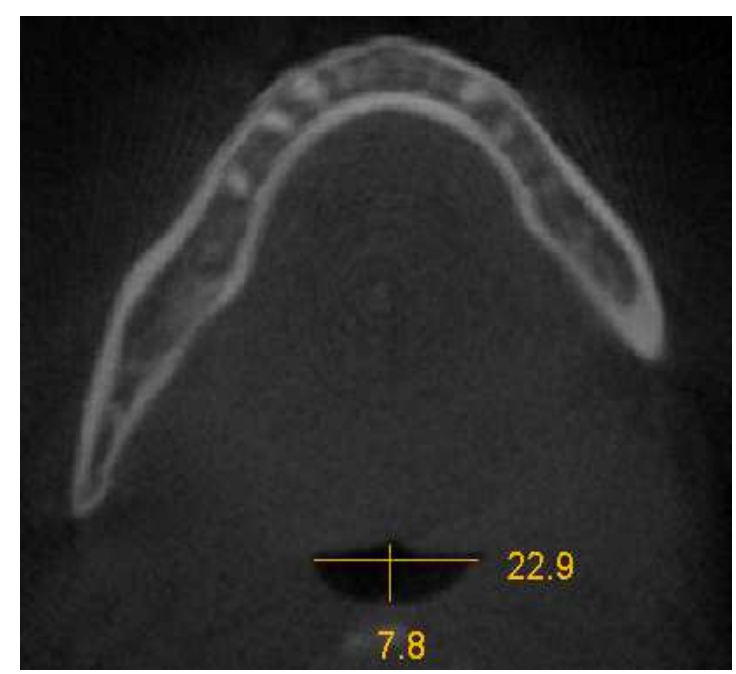

Abb. 27: axiale Vermessung auf Höhe TPW 


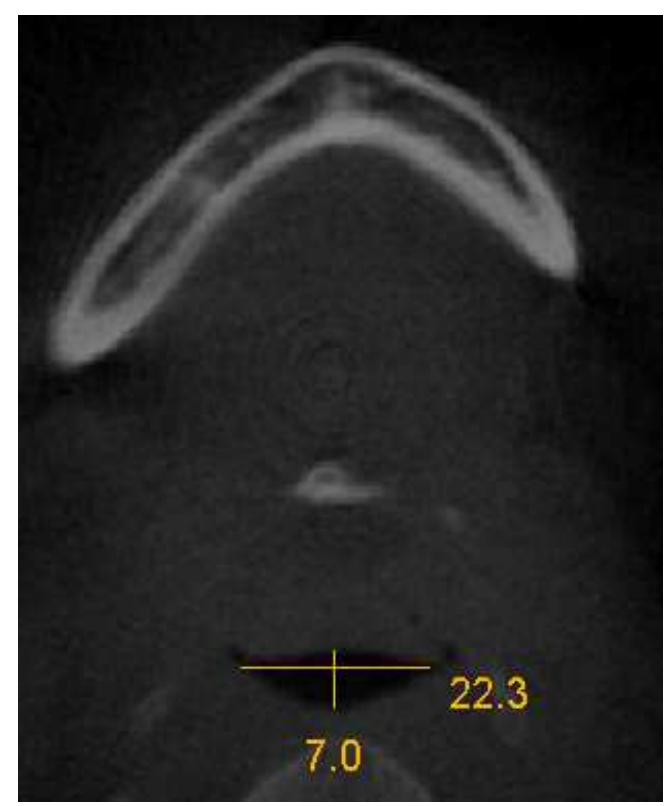

Abb. 28: axiale Vermessung auf Höhe LPW

Die Abbildungen 25 bis 28 zeigen die axiale Vermessung auf der jeweiligen Höhe.

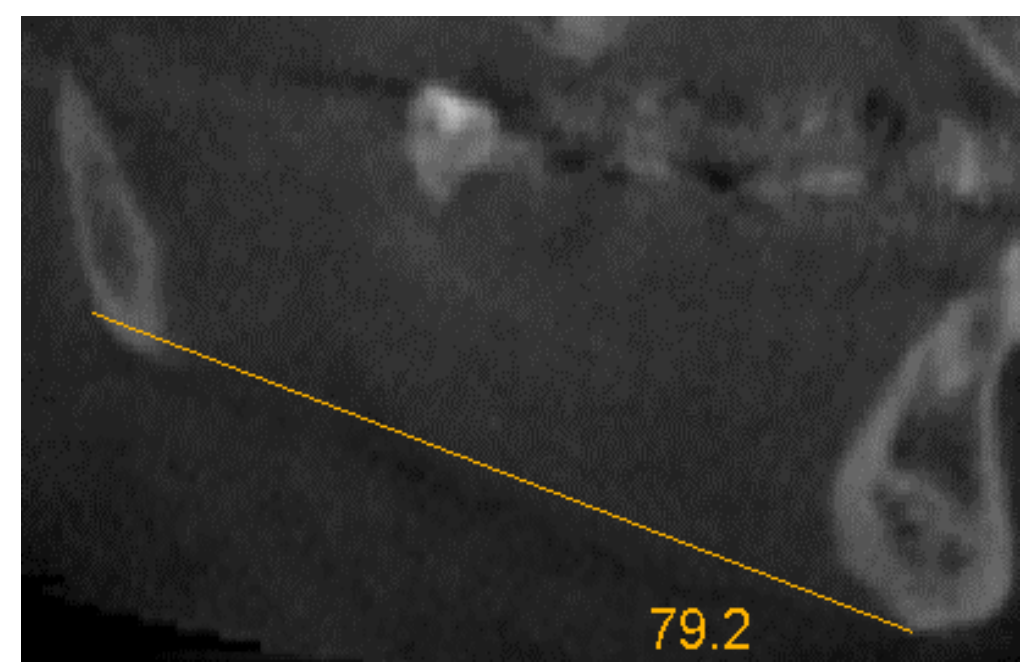

Abb. 29: Vermessung der Unterkieferlänge

Die Abbildung 29 stellt die Vermessung der Unterkieferlänge dar. 


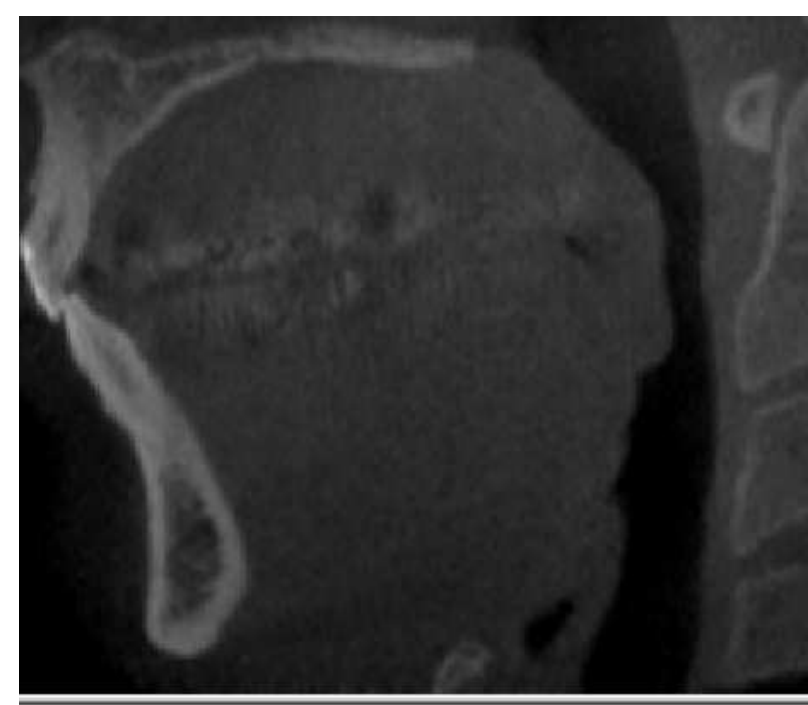

Abb. 30: mediosagittaler Schnitt (1)

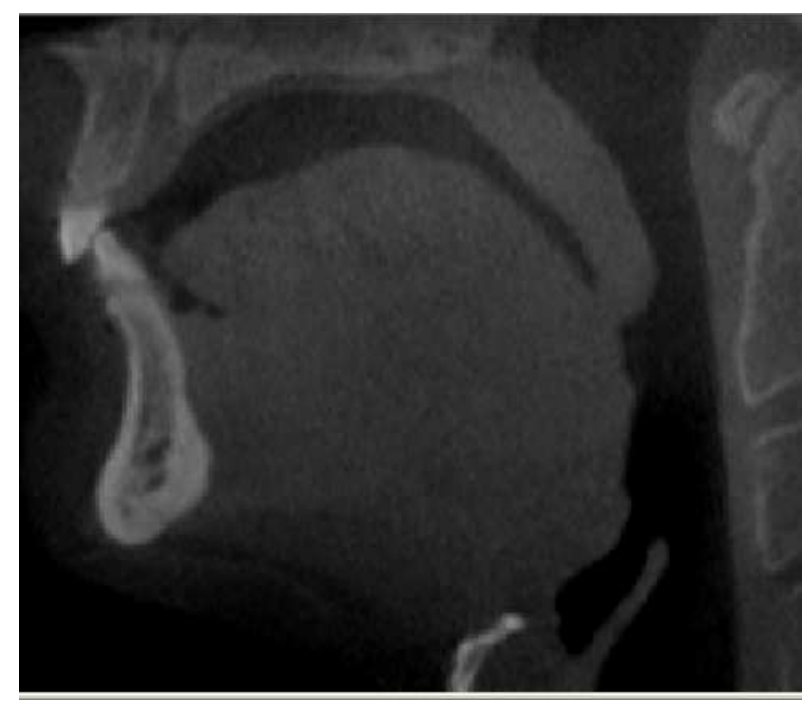

Abb. 32: mediosagittaler Schnitt (3)

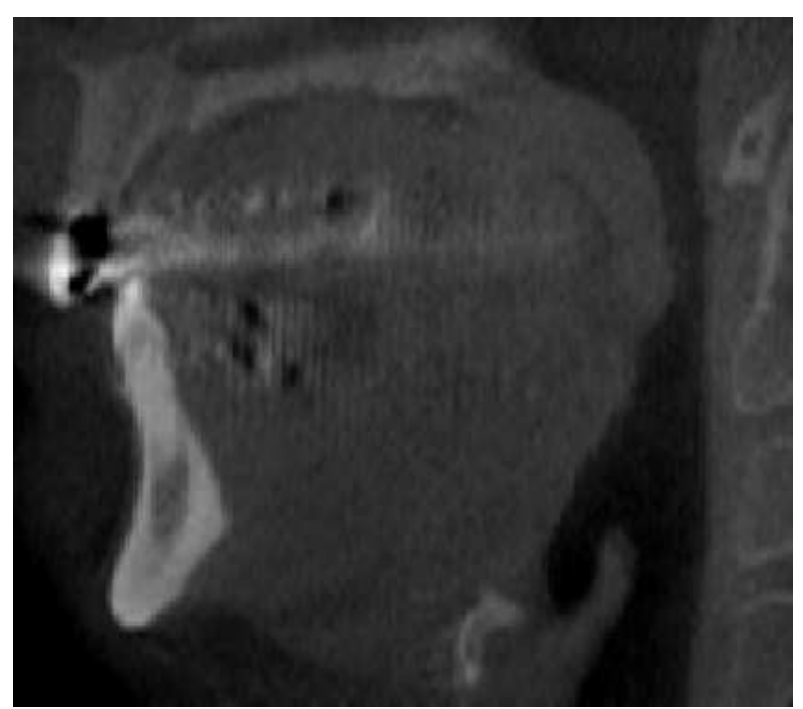

Abb. 31: mediosagittaler Schnitt (2)

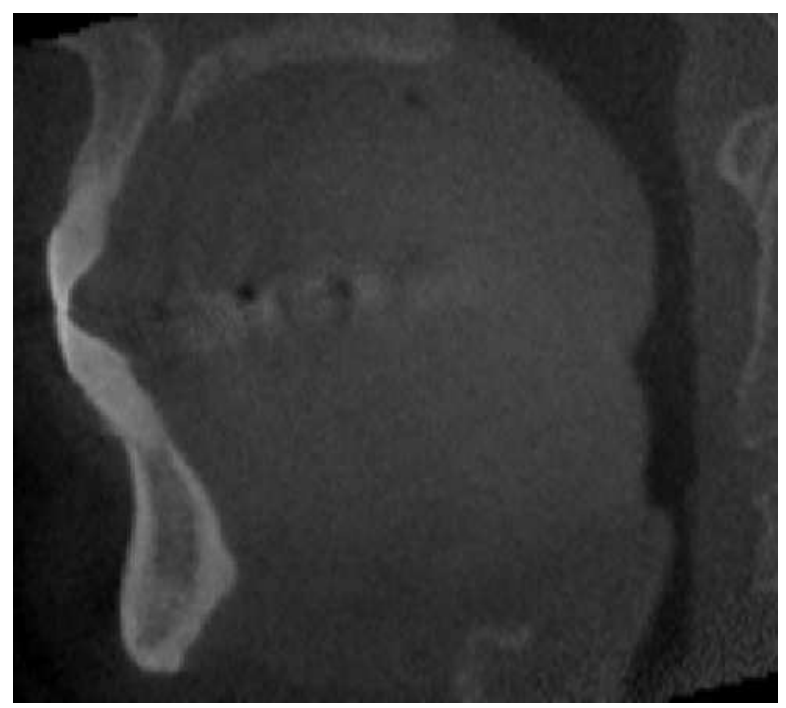

Abb. 33: mediosagittaler Schnitt (4)

Die Abbildungen 30 bis 33 zeigen die mediosgittalen Schnitte verschiedener Patienten. In der Abbildung 32 erkennt man einen offenen Systemzustand, in der Abbildung 33 dagegen einen geschlossenen Systemzustand. In den Schnitten 30, 31 und 33 ist eine Differenzierung zwischen weichen Gaumen und Zunge nicht möglich. In den Abbildungen 30 und 33 liegt die Epiglottisspitze dem dorsalen Zungengrund an und lässt sich somit nur schwer identifizieren, in der Abbildung 33 ist zusätzlich keine Vallecula erkennbar. 


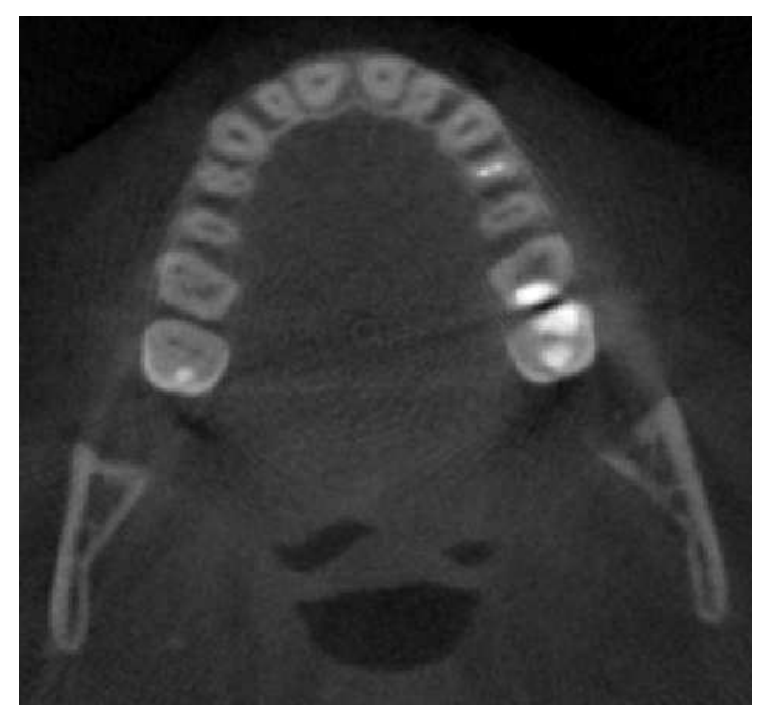

Abb. 34: axialer Schnitt; offener Funktionszustand

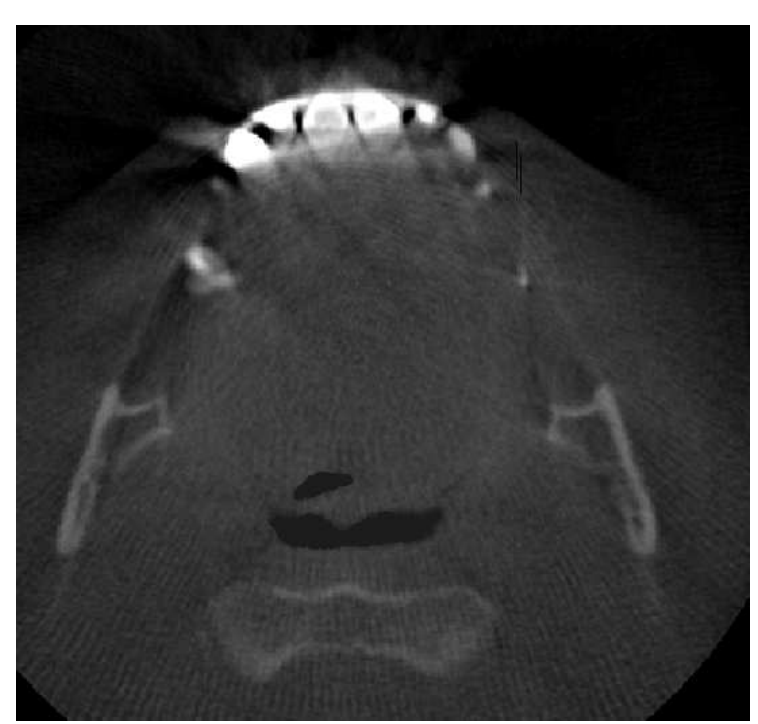

Abb. 36: axialer Schnitt; Isthmus 5,5*27,8 mm

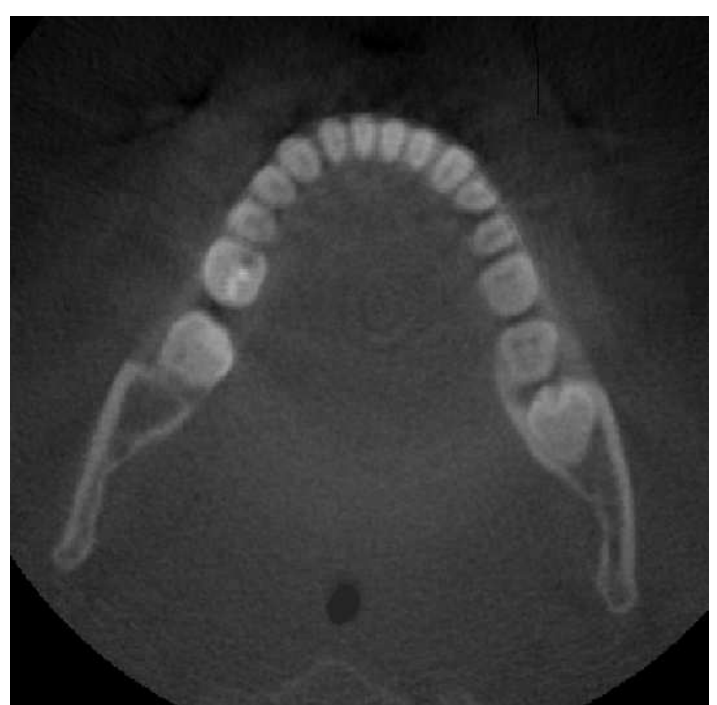

Abb. 35: axialer Schnitt; Isthmus 5,3*5,5 mm

Abb. 35: axialer Schnitt; Isthmus $5,3 * 5,5 \mathrm{~mm}$ 


\section{Diskussion}

In verschiedenen radiologischen Studien wurde der Zusammenhang zwischen der Anatomie des orofazialen Systems und Rhonchopathien untersucht. Die meisten Studien wurden mit Hilfe des Fernröngtenseitenbildes (Johal et al. 2007, Oosterkamp et al. 2007, Battagel et al. 2002, Battagel et al. 2000, Pradel et al. 2000, Battagel und L’Estrange 1996, Pracharktam et al. 1996, Solow et al. 1996, Tangugsorn et al. 1995, Hochban et al. 1994, Partinen et al. 1988) durchgeführt. Wie Riley et al. (1986) schon anmerkten, kann dieses Verfahren lediglich zweidimensionale Bilder liefern.

Des Weiteren stehen Schichtbildverfahren wie die Computertomographie (Koren et al. 2009, Fairburn et al. 2007, Fajdiga 2005, Yucel et al. 2005, Pepin et al. 1999, Avrahami und Englender 1995) und die Magnetresonanztomographie (Hora et al. 2007, Horner et al. 1989) zur Verfügung.

Durch diese Studien wurden weitreichende Erkenntnisse über den Einfluss der Anatomie auf schlafbezogene Atemstörungen gewonnen. Die aus diesen Studien gewonnenen Ergebnisse sind schwer miteinander zu vergleichen, da die Aufnahmebedingungen, Messstrecken und Bezugsebenen variieren. Bisher konnten Zahnmediziner nur mit Hilfe des Fernröntgenseitenbildes den Pharynx zweidimensional bildlich betrachten. Mit dem DVT ist es nun auch dem Zahnmediziner seit 1997 möglich, den Pharynx dreidimensional darzustellen. Da die Durchführung und Befundung dieses neuen Verfahrens durch den zahnärztlichen Behandler selbst erfolgen kann, hat der DVT in den letzten Jahren zunehmende Verbreitung gefunden (Danforth 2003). Ein Schwerpunkt dieser Studie war es, dieses Verfahren hinsichtlich seiner Bedeutung zur Ermittlung prädiktiver anatomischer Parameter bezüglich Rhonchopathien und OSAS zu untersuchen. 


\subsection{Kritische Betrachtung der angewandten Methode}

\subsubsection{Aspekte der Patientenauswahl}

Das Patientenkollektiv umfasste 104 Patienten im Alter von 30 bis 68 Jahren, das durchschnittliche Alter betrug 46,6 Jahre \pm 11,28 Stdabw.. Die Patienten waren beiderlei Geschlechts (52 Männer mit einen Durchschnittsalter von 46,82 Jahre \pm 11,25 Stdabw. und 52 Frauen mit einem Durchschnittsalter von 46,48 Jahre \pm 11,32 Stdabw.) mit ausgefülltem Schlaffragebogen.

Damit lag diese Studie im Vergleich zu Johal et al. (2007) (Durchschnittsalter 48,6 Jahre \pm 8,3 Stdabw. im Alter zwischen 27 und 66 Jahren), Solow et al. (1996) (Durchschnittsalter 50 Jahre \pm 9,4 Stdabw., im Alter zwischen 29 uns 70 Jahre), Tangugsorn et al. (1995) (Durchschnittsalter 36,8 Jahre, im Alter zwischen 28 und 45 Jahren und Lowe et al. (1986) (Durchschnittsalter 48 Jahre \pm 9,4 Stdabw.) mit dem Durchschnittsalter leicht unterhalb der genannten Studien.

\begin{tabular}{|l|l|l|}
\hline Lowe et al. (1986) & 25 Männer & \\
\hline Tangugsorn et al. (1995) & 136 Männer & \\
\hline Solow et al. (1996) & 153 Männer & 8 Frauen \\
\hline Pradel et al. (2000) & 69 Männer & \\
\hline Battagel et al. (2000) & 115 Männer & 21 Frauen \\
\hline Johal et al. (2007) & 78 Männer & 18 Frauen \\
\hline Koren et al. (2009) & 47 Männer & 18 Frauen \\
\hline Kim et al. (2010) & 75 Männer & \\
\hline
\end{tabular}

Tab. 15: Gruppengröße und Geschlechtsverteilung anderer Studien

Wie aus der Tabelle 15 zu erkennen ist, wurden oft nur männliche Probanden bzw. Gruppen mit überwiegend männlichen Probanden untersucht.

Das Patientenkollektiv ist nur eingeschränkt repräsentativ, da nur Patienten zwischen 30 und 68 Jahren, mit gesicherter Okklusion und Schneidezähnen berücksichtigt wurden. Weiterhin wurden nur DVT-Datensätze verwendet, bei denen das Aufnahmefeld die zu vermessenen Punkte abbildete. 
In dieser Studie lag der Anteil OSAS-bestätigter Patienten bei 7,7\% (8 Patienten). Damit liegt der Wert deutlich über dem von Peter et al. (1990) angegebenen Wert, die die Prävalenz in der Bundesrepublik Deutschland auf 1 - 3\% geschätzt haben. Ähnliche Werte wurden auch von anderen Autoren für weitere Länder angegeben (Young 2002, Bixler et al. 2001, Gislason et al. 1988, Lavie 1983).

Nach Partinen und Telakivi (1992) ist die Prävalenz beim männlichen Geschlecht zwischen 40 und 65 Jahren am höchsten und kann 8,5\% oder mehr betragen.

\subsubsection{Aspekte der Fragebogenauswertung}

Mit Hilfe des Fragebogens war es ohne apparativen Aufwand möglich, Daten über das Schlafverhalten der Patienten zu ermitteln. Aussagen zur Diagnose des OSAS hatten hohe Relevanz, die Angaben zur Epworth Sleepiness Scale (ESS) und zum Schnarchverhalten mussten kritisch beurteilt werden.

Um Tendenzen sichtbar zu machen, wurde das Kollektiv anhand des Fragebogens in drei Gruppen aufgeteilt und jeweils die Mittelwerte angegeben (Tab. 2). Der ESS-Grenzwert $\geq 9$ für die erste Gruppe wurde in Anlehnung an Johal et al. (2007) gewählt.

Eine weitere Unterteilung des Kollektives für die statistische Auswertung in eine OSASGruppe und Kontrollgruppe unter Verwendung des ESS-Wertes erschien als nicht geeignet, da die Angaben auf subjektiven Empfindungen des Patienten basierten und aufgrund der daraus resultierenden kleinen Gruppengrößen die Aussagekraft einschränkt würde.

Der ESS-Fragebogen ist als Screening-Verfahren bezüglich des OSAS als gut zu beurteilen, doch führt er aber auch dazu, dass OSAS-Patienten in Behandlung durch dieses Raster fallen können und somit zu einem falsch negativen Ergebnis führen. Teilweise wurden ESS-Werte von 1 bzw. 2 (Tab. 3) von Patienten mit bestätigtem OSAS angegeben. Dies könnte an mangelnder Compliance oder geringer Symptomatik aufgrund bereits bestehender Behandlung der Patienten liegen, deshalb sind auch die folgenden Korrelationen mit dem ESS-Wert kritisch zu betrachten.

Fazit: Der ESS-Wert ist ohne Informationen über vorherige Diagnose und eventuelle Behandlung vom OSAS aus den oben genannten Gründen nicht für eine Differenzierung des Kollektives geeignet, vielmehr sollten Werte aus dem Schlaflabor verwendet werden.

Andere Studien unterteilten das Patientenkollektiv nach dem AHI, der im Schlaflabor mittels Polysomnographie bestimmt wurde. Dabei waren aber die definierten Grenzen für die Gruppeneinteilung sehr unterschiedlich. Pradel et al. (2000) definierten die OSAS-Patienten 
ab einem AHI-Wert >20 pro Stunde, Battagel et al. (2000) legten die Grenze bei einem AHI von 15 pro Stunde fest, Enciso et al. (2010) bei einem AHI-Wert $\geq 10$ und Johal et al. (2007) nahmen einen Grenzwert von AHI >5 pro Stunde an.

\subsubsection{Technische Aspekte des DVT}

Da das verwendete DVT ein anatomisches Volumen in Zylinderform mit den Abmessungen $15 \times 15 \mathrm{~cm}$ abbildete, ist nur bei bestimmten Indikationen eine Darstellung des kompletten Pharynx möglich. Besonders bei Indikationen, die die Kieferhöhlen betrafen, war die Darstellung des Hypopharynx und des Zungenbeins nur selten möglich. Bei der Nutzung des CT besteht diese Einschränkung nicht, da die Größe des Scanbereiches individuell für den Patienten angepasst werden kann. Beim Fernröntgenseitenbild erfasst die Größe des Aufnahmefeldes den gesamten Schädel mitsamt des Hypopharynx und des Zungenbeins.

Durch die Aufnahmedauer beim NewTom QR-9000 von 76 Sekunden lassen sich Bewegungsunschärfen nicht vermeiden, die durch Bewegungen des Patienten entstehen, z.B. beim Ein- und Ausatmen. Caballero et al. (1998) haben in einer CT-Studie nachgewiesen, dass sich das Pharynxvolumen mit der Atmung verändert. Schwenzer und Ehrenfeld (2000) wiesen darauf hin, dass insbesondere bei Prozessen des Zungengrundes und des Pharynx, die Untersuchungszeiten möglichst kurz sein sollten, da wiederholtes Schlucken oder Bewegungen des Patienten zu erheblichen Artefakten in der Bildgebung führen.

Im Vergleich zum CT ist die Aufnahmezeit im verwendeten DVT länger. Für ein Spiral-CT vom OK (37 mm) werden 19,3 Sekunden, für ein UK-Spiral-CT (27 mm) werden 14,3 Sekunden und für ein konventionelles Fernröntgenseitenbild werden 0,5 Sekunden benötigt (Pasler und Visser 2000). Kim et al. (2010) stellten den Pharynx mittels CT dar (pro Schicht 0,4 Sekunden). Holberg et al. (2005) verglichen die Darstellungsqualität des DVT NewTom QR-9000 mit einem Dental-CT. Nach Holberg et al. (2005) traten bei der digitalen Volumentomographie in $20 \%$ der Fälle relevante Bewegungsartefakte auf, die aus der relativ langen Aufnahmezeit von 76 Sekunden resultierten, während im Dental-CT diese Artefakte bei einer Aufnahmezeit von 1,1 Sekunden pro axialer Schicht nicht zu beobachten waren. Ein wesentlicher Unterschied zwischen beiden radiologischen Verfahren besteht darin, dass sich Kopf-, Kiefer- und Atembewegungen des Patienten bei der DVT auf die Qualität des gesamten Volumendatensatzes auswirken, während bei der Dental-CT lediglich eine oder wenige axiale Schichten (spiralförmige Aufnahme) betroffen sind. 
Bei neueren DVT konnte die Aufnahmezeit verkürzt werden, wodurch das Auftreten und die Ausprägung der Bewegungsunschärfen nachgelassen hat. Laut Cacaci et al. (2007) beträgt die Aufnahmezeit bei dem DVT 3D eXam von KaVo nur 8,5 Sekunden für ein 23x17 cm großes Aufnahmevolumen. Auch durch die Entwicklung ultraschneller Sequenzen (5 Bilder pro Sekunde) gewinnt die MRT zunehmend für die Diagnostik des OSAS an Bedeutung (Schwenzer und Ehrenfeld 2000).

Durch die Möglichkeit der 3D-Darstellung mittels DVT sind Summationseffekte im Gegensatz zum zweidimensionalen Fernröntgenseitenbild ausgeschlossen. Ein weiterer Vorteil eines digitalen Volumendatensatzes ist, dass es möglich ist, verschiedene Schnitte in der sagittalen, koronalen und axialen Ebene zu berechnen. Dadurch ist eine dreidimensionale Vermessung des orofazialen Systems möglich. Durch verschiedene Schnitte und das Fehlen von Summationseffekten war eine genauere Bestimmung der Messpunkte möglich.

Lenza et al. (2010) stellten klar, der Pharynx könne nicht anhand von einzelnen linearen Messpunkten wie beim Fernröntgenseitenbild genau beschrieben werden. Eine 3D-Analyse ergebe ein besseres Bild von den anatomischen Charakteristika des oberen Luftweges und könne dadurch entscheidend für die Diagnostik sein.

Da der Kontrast zwischen Luft und Weichgeweben ausreichend gut im DVT dargestellt wurde, konnte im Gegensatz zu Johal et al. (2007), Battagel et al. (2000), Battagel und L`Estrange (1996), Pracharktam et al. (1996) und Lowe et al. (1985), welche die Vermessung mittels Fernröntgenseitenbild durchgeführt haben, auf Bariumsulfat als Kontrastmittel verzichtet werden.

Ein Nachteil des DVT gegenüber dem CT und MRT ist der geringe Kontrast zwischen den Weichgewebsarten. Dadurch ließen sich Weichgewebe nicht voneinander differenzieren, was die Identifizierung von Messpunkten erschwerte. Auch Holberg et al. (2005) wiesen daraufhin, dass eine Weichteildifferenzierung mit dem DVT nicht möglich ist. Bei Kontakt zweier Weichgewebe sind die Begrenzungen der jeweiligen Gewebe im Bereich der Kontaktfläche nicht zu erkennen. Im Gegensatz zum DVT werden im CT sogenannte Hounsfield-Einheiten (HE) angegeben. Da diese Werte charakteristisch für die jeweiligen Gewebe des menschlichen Körpers sind, können dadurch Gewebe differenziert werden. Auch im MRT ist es möglich, die Gewebearten zu differenzieren. So bestimmten Hora et al. (2007) und Horner et al. (1989) die Dicke des parapharyngealen Fettgewebes. 
Hartgewebe, wie Knochen und Zähne, lassen sich im DVT, wie auch im Fernröntgenseitenbild, im CT und im MRT, gut vom Weichgewebe differenzieren, dadurch war eine exakte Identifizierung der knöchernen Messpunkte gegeben.

Allerdings riefen Metallrestaurationen Artefakte hervor, die das Auffinden der Messpunkte beeinträchtigten, z.B. des unteren Inzisialpunktes bei Überkronung der oberen Frontzähne. Holberg et al. (2005) stellten einen Unterschied in dem Ausmaß der Artefaktbildung an Zähnen mit Metallfüllungen fest. Metallartefakte, die zu einer wesentlichen Beeinträchtigung der Abbildungsqualität führten, traten bei dem DVT bei keinem Zahn mit Metallfüllung auf, während beim Dental-CT alle Zähne mit Metallfüllungen ausgeprägte Metallartefakte zeigten, die eine adäquate Beurteilung des betroffenen Zahnes unmöglich machten.

Die Voxelgröße des DVT NewTom QR-9000 betrug 0,25 mm. Es wurde eine Auflösegenauigkeit innerhalb der Messebenen von der doppelten Pixelgröße, also 0,5 mm, angenommen. Die Ablesegenauigkeit am Bildschirm wurde durch die Pixelgröße limitiert, obwohl die Software die Messwerte in 0,1 mm Schritten angab. Aus diesem Grund war die Ablesegenauigkeit als nachrangig zu betrachten. Anbieter neuer DVT (z.B. PaX-Reve3D von der Firma Orangedental) werben mit Voxel-Größen zwischen 0,08 mm und 0,16 mm.

Bei der Vermessung von Pinlängen mit dem DVT NewTom QR-9000 stellte Hübinger (2008) Abweichungen im Durchschnitt von 0,020 mm für das DVT und 0,194 mm für das CT fest. Bei diesem Vergleich sind die Ungenauigkeiten der CT-Aufnahmen also am größten, wo hingegen die Vermessung durch das DVT am genauesten erscheint. Auch bei der Vermessung des Abstandes vom Canalis mandibulae bis zum krestalen Rand der Mandibula zeigte das DVT im Durchschnitt eine kleinere Abweichung (DVT -0,076 mm) gegenüber dem CT (0,168 mm) (Hübinger 2008). Stratemann et al. (2008) konnten in einer ähnlichen Untersuchung mit dem DVT NewTom QR-9000 eine durchschnittliche Abweichung von 0,07 mm von der Realität in der Sagittalebene nachweisen. Goch (2005) stellte bei der Messgenauigkeit am menschlichen Unterkiefer zwischen dem CT und dem DVT NewTom QR-9000 keine signifikanten Unterschiede fest. Für die CT-Untersuchungen ergaben sich im Mittel Abweichungen von -0,23 mm bei $100 \mathrm{~mA},-0,27 \mathrm{~mm}$ bei $50 \mathrm{~mA}$ und $-0,24 \mathrm{~mm}$ bei 10 mA. Bei DVT-Untersuchungen lag die mittlere Abweichung bei -0,22 mm. Laut Cohnen et al. (2002) lag die metrische Genauigkeit bei der DVT zwischen -0,7 und +0,6 mm und ist vergleichbar mit den Ergebnissen der Computertomographie. 
Der DVT NewTom QR-9000 führte zu einer geringeren Strahlenbelastung als ein CT, aber zu einer höheren Strahlenbelastung als ein Fernröntgenseitenbild. Der MRT verursacht keine Strahlenbelastung für den Patienten.

Im Vergleich zur allgemeinen Radiologie schätzte Dücker (2006) die Strahlenbelastungen in der Zahnmedizin als sehr gering ein (z.B. CT Becken $8000 \mu \mathrm{Sv}$, Fernröntgenseitenbild $2 \mu \mathrm{Sv}$, DVT $36 \mu \mathrm{Sv}$, Dental CT 150-350 $\mu \mathrm{Sv}$, jeweils effektive Dosis).

Benz et al. (2003) drückten in ihren Vergleichen die Strahlenbelastung in Tagen aus. Hierbei wählten sie als Vergleichsgrundlage die tägliche Strahlenbelastung, die ein Bundesbürger aus natürlichen Quellen erhält (2,1 mSv pro Jahr). Insgesamt nimmt ein Bundesbürger statistisch $4 \mathrm{mSv}$ effektive Dosis pro Jahr auf. Benz et al. (2003) arbeiteten mit der effektiven Dosis, um Ganz- und Teilkörperexpositionen vergleichen zu können.

\begin{tabular}{|l|l|r|r|}
\hline Röntgengerät & Körperbereich & entsprechend in Tagen & effektive Dosis in mSv \\
\hline CT & OK & 36,80 & 0,21 \\
\hline CT & UK & 23,75 & 0,14 \\
\hline DVT & OK + UK & 54,50 & 0,32 \\
\hline FRS analog & Schädel & 0,35 & 0,002 \\
\hline
\end{tabular}

Tab. 16: Strahlenbelastung in der Zahnmedizin (modifiziert nach Benz et al. 2003, Seite 17)

Pasler und Visser (2000) gaben eine effektive Dosis von $126 \mu$ Sv für ein OK- und UK-SpiralCT (120 kV/80 mA/64 mm Spirale) an, dabei wurde an der Parotis eine Energiedosis von 1,566 mGy ermittelt. Die Autoren gaben für das konventionelle Fernröntgenseitenbild eine effektive Dosis von 2,3 $\mu \mathrm{Sv}$ und eine Energiedosis an der Parotis von 0,103 mGy an.

Schnelle (2001) verglich die Strahlenbelastung des NewTom QR-9000 mit einem CT anhand von Organdosiswerten, die an einem Phantomkopf gemessen wurden.

\begin{tabular}{|l|l|r|}
\hline \multicolumn{1}{|c|}{ Röntgengerät } & \multicolumn{1}{|c|}{ Körperbereich } & $\begin{array}{c}\text { Äquivalentdosis } \\
\text { in mSv }\end{array}$ \\
\hline DVT NewTom QR-9000 & Parotis links & 1,4 \\
\hline & Regio OK 6er links & 3,3 \\
\hline CT 140kV/94mA & Parotis links & 2,9 \\
\hline & Regio OK 6er links & 22,1 \\
\hline
\end{tabular}

Tab. 17: Strahlenbelastung DVT/CT (Auszug aus Schnelle 2001, Seite 25) 
Cohnen et al. (2002) nahmen Dosismessungen bei CT- und DVT-Untersuchungen an einem Phantomkopf vor. Die erreichte Ortsdosis lege beim DVT mit 1,5 mGy für die Augenlinse und mit 0,9 mGy für die Schilddrüse in der Größenordnung einer Niedrigdosis-CTUntersuchung.

Nach Herstellerangaben liegt unter klinischen Bedingungen die Röhrenstromstärke bei dem DVT NewTom QR-9000 je nach Knochenvolumen und -dichte zwischen 0,8 und 15 mA und die durchschnittliche Strahlenbelastung bei $6 \mathrm{mSv}$ pro Aufnahme (Goch 2005). Pepin et al. (1992) führten eine CT-Untersuchung zum Vermessen des oberen Luftweges durch. Dafür wurden pro Patient 8 bis 10 Schichten jeweils $5 \mathrm{~mm}$ dick angefertigt. Jede Schicht führte zu einer Dosis an der Hautoberfläche von 10 mGy. Laut Kistler (2007) lag die Strahlendosis bei der Aufnahme mit dem DVT-Gerät Galileus (Sirona, Bensheim) ungefähr im Bereich der analogen Panoramaaufnahmen.

Die geringe Strahlenbelastung des DVT wird immer wieder als Hauptvorteil gegenüber dem Dental-CT angeführt (Hümmeke et al. 2003). Mit der DVT-Aufnahmetechnik ist eine Reduktion der Strahlenbelastung ohne große Verluste der Darstellungsgenauigkeit im Vergleich zum konventionellen CT möglich (Rother 2006). So kann eine bis zu 15-fache Reduktion der CT-Dosiswerte durch die neue Technik erreicht werden (Scarfe et al. 2006). Nach Mozzo et al. (1998) beträgt die Strahlendosis nur etwa ein Sechstel einer herkömmlichen Computertomographie.

In den letzten Jahren wurden jedoch auch für das Dental-CT Low-Dose-Protokolle entwickelt (Thiel und Hassfeld 2001), die über eine Verringerung des Röhrenstroms eine Reduktion der Strahlenbelastung bei gleicher Abbildungsqualität erzielen sollen. Trotz dieser erfolgreichen Reduktion der Strahlenbelastung beim Dental-CT ist diese bei dem DVT noch etwas geringer (Möbes et al. 2000, Möbes et al. 1999).

Möbes et al. (2000) konstatierten, dass die effektive Dosis bei der digitalen Volumentomographie im Bereich von Niedrigdosis-CT-Scans mit 43 mA liege. Suomalainen et al. (2008) stellten hierzu Versuche an, die Dosiswerte des Multislice-CT den Werten des DVT anzugleichen. Das Resultat war eine signifikante Verschlechterung der Darstellungsgenauigkeit vom Multislice-CT gegenüber dem DVT. Auch andere Autoren konnten durch Reduktion der Röhrenstromstärke beim CT das Erreichen vergleichbarer DVTDosiswerte nachweisen, was jedoch auch hier den nachteiligen Effekt der Verschlechterung der Bildqualität mit sich brachte (Schnelle 2001).

Abschließend lässt sich sagen, dass die bisher durchgeführten Studien zur Strahlenexposition sich nur schwer miteinander vergleichen lassen, da mit unterschiedlichen Geräte und 
Einstellungen gearbeitet wurde. Eine Verkleinerung des geröntgen Volumens, leistungsfähigere Software, reduziertes Auflösungsvermögen und verminderter Kontrast bewirken eine geringere Strahlenbelastung. Oft werden die ermittelten Strahlendosen in unterschiedlichen Einheiten angegeben, was einen Vergleich erschwert.

\subsubsection{Untersuchungsbedingungen}

Für die DVT-Aufnahmen wurde eine reproduzierbare Position der Patienten gewählt. Die Patienten lagen auf dem Rücken und der Kopf war in einer Schale gelagert. Dabei bestand Okklusionskontakt zwischen den Zähnen und der Mund war geschlossen. Solow et al. (1996) stellten fest, dass, wenn der Kopf extendiert wird, sich der Pharynx, besonders der Hypo- und Mesopharynx erweitert. Deshalb wurde während dieser Studie immer die gleiche Kopfschale verwendet. Durch die Rückenlage wurde die Schlafposition nachgeahmt, sodass die anatomischen Verhältnisse des Pharynx während der Aufnahme annähernd mit den anatomischen Verhältnissen während des Schlafes übereinstimmten. Ingman et al. (2004) konnten schwerkraftbedingte Veränderungen des Luftweges zwischen liegender und aufrechter Aufnahmeposition nachweisen. Im Fernröntgenseitenbild zeigte sich in der liegenden Aufnahmeposition eine Einengung des Mesopharynx und eine minimale Verdickung des weichen Gaumens, aber keine signifikanten Veränderungen im Naso- und Hypopharynx. Somit sind die Ergebnisse dieser Studie nur eingeschränkt mit Studien mit aufrechter Aufnahmeposition vergleichbar. Bei den zurzeit auf dem Markt angebotenen DVT werden die Patienten überwiegend in der aufrechten Position geröngt, was dann ggf. bei der Auswertung mit bedacht werden muss.

Weiterhin war für die Aufnahmen die konkrete Anweisung der Röntgen-TechnischenAngestellten wichtig, um Positionierungsfehler zu verringern. Die Patienten wurden vor der Aufnahme durch die Röntgen-Technischen-Angestellten angewiesen, die Zähne in Okklusionskontakt zu bringen (wie auch bei Johal et al. 2007, Pracharktam et al. 1996, Tangugsorn et al. 1995) und zu schlucken (wie auch bei Oosterkamp et al. 2007, Pepin et al. 1999, Solow et al. 1996, Lowe et al. 1985), damit in der Mundhöhle ein negativer Druck entsteht und es somit zu einer Anlagerung der Zunge an den Gaumen kommt. Trotz der Instruktion durch das Personal platzierten gelegentlich die Patienten die Zunge nur im vorderen Bereich an den Gaumen. Gründe für die falsche Positionierung können sein, dass die Patienten die Anweisungen falsch verstehen oder dass die Patienten den Unterdruck während der gesamten Aufnahmezeit von 76 Sekunden nicht aufrecht halten können. Dadurch können Messungen in diesem Bereich durch Bewegungsartefakte verfälscht worden sein. Zusätzlich 
sind dadurch die Ergebnisse untereinander schwer vergleichbar, da man davon ausgeht, dass die Patienten vor der Aufnahme geschluckt haben und somit anatomische Verhältnisse nach dem Schlucken vorfindet. Somit ist erkennbar, dass auch trotz richtiger Instruktion es keine Garantie für eine fehlerfreie Mitarbeit des Patienten gibt. Um dies auszuschließen, wäre es sinnvoll, die Aufnahme unter Kontrolle einer Membrantrichterplatte durchzuführen. Mit einer druckanzeigenden Membrantrichterplatte kann objektiv überprüft werden, ob ein Unterdruck in der Mundhöhle besteht und die Zunge eine Gaumenkontaktposition einnimmt (Engelke et al. 2006).

Bei anderen Studien, vor allem bei denen mittels Fernröntgenseitenbild, wurde eine natürliche, aufrechte Kopfposition (Johal et al. 2007, Pracharktam et al. 1996, Solow et al. 1996, Tangugsorn et al. 1995, Lowe et al. 1985) oder eine Kopfposition im Cephalostat (Battagel et al. 2000, Pradel et al. 2000, Hochban et al. 1994, Partinen et al. 1988) gewählt, die aber nicht die Schlafposition der Patienten widerspiegeln und es dadurch zu einer verfälschten Darstellung des Pharynx kommt.

Bei den CT-Studien von Koren et al. (2009), Fairburn et al. (2007), Fajdiga (2005), Yucel et al. (2005), Pepin et al. (1999), Avrahami und Englender (1995), Galvin et al. (1989) und Bohlman et al. (1983) war die Lagerung ähnlich wie bei dem DVT, sodass man die Messwerte miteinander vergleichen kann. Gleiches gilt auch für die MRT-Studien z.B. von Hora et al. (2007) und Baik et al. (2002).

Aufgrund der Lautstärke der Geräte und der Anweisungen der Röntgen-TechnischenAngestellten befanden sich die Patienten stets im Wachzustand, dabei wird ein geringer Pharynxdurchmesser durch einen erhöhten Muskeltonus kompensiert. Doch während des Schlafes verringert sich der Muskeltonus und die Luftwegsdimensionen nehmen ab, bzw. die Pharynxwände verlieren ihre Steifheit und können so leichter durch den Unterdruck während der Inspirationsphase kollabieren.

\subsubsection{Datenerhebung und -auswertung}

Die Reproduzierbarkeit der Datenerhebung wurde durch die Nutzung der Nasallinie als Referenzebene gesteigert, zusätzlich wurde dadurch ein exakter Vergleich der Messwerte zwischen den Patienten ermöglicht.

Die Referenzebene war eindeutig und auch bei zahnlosen Patienten zu identifizieren. Aus dem Rohdatensatz wurden die $1 \mathrm{~mm}$ dicken axialen Schnitte parallel zu Nasallinie berechnet, wodurch reproduzierbare und vergleichbare Schnitte entstanden, um den Luftweg zu 
vermessen. Auch Fairburn et al. (2007) haben bei ihren Messungen mit dem CT die axialen Schnitte parallel zur Nasallinie ausgerichtet.

Solow et al. (1996) merkten an, dass viele Studien skelettale Referenzpunkte nutzen, um Linien zu definieren, um anhand dieser den Luftweg zu vermessen. Dabei würde aber nicht unbedingt der geringste Durchmesser des Luftweges gemessen. Pradel et al. (2000), Hochban et al. (1994) und Lowe et al. (1985) benutzten Hilfslinien (Mandibularlinie, Okklusionsebene), um auf deren Verlängerung den Pharynx zu vermessen. Aufgrund der Abhängigkeit der Mandibularlinie und Okklusionsebene von der Bezahnung und Kopfhaltung des Patienten ist die Neigung dieser Linien zum Luftweg variabel, somit sind die Messwerte nur eingeschränkt auf andere Patienten übertragbar.

Andere Studien maßen nicht parallel zu einer Referenzebene, sondern die geringsten sagittalen Abstände zwischen der Pharynxhinterwand und bestimmten Punkten wie z.B. der Uvulaspitze (Oosterkamp et al. 2007, Pradel et al. 2000, Battagel und L'Estrange 1996, Solow et al. 1996, Tangugsorn et al. 1995, Hochban et al. 1994), der kleinsten Stelle hinter dem weichen Gaumen (Isthmus) (Johal et al. 2007, Teitelbaum et al. 2007, Battagel et al. 2000, Solow et al. 1996), hinter der Zunge (Johal et al. 2007, Teitelbaum et al. 2007, Battagel et al. 2000, Battagel und L’Estrange 1996, Solow et al. 1996) oder auf Höhe der Vallecula (Oosterkamp et al. 2007, Solow et al. 1996, Tangugsorn et al. 1995, Hochban et al. 1994). Nachteilig bei der oben genannten Methode ist, dass zwei Messpunkte pro Messstrecke definiert werden müssen, was $\mathrm{zu}$ einer erhöhten Fehleranfälligkeit führt und die Reproduzierbarkeit negativ beeinflusst.

$\mathrm{Da}$ in dieser Studie parallel $\mathrm{zu}$ einer Referenzebene gemessen wurde, war für die Streckenmessung nur die Bestimmung des anterioren Messpunktes nötig, die Position des posterioren Punktes an der hinteren Pharynxwand wurde durch die Nasallinie als Referenzebene vorgegeben. Dadurch wurde eine Standardisierung erreicht, die einen Vergleich von Messwerten verschiedener Patienten ermöglicht. Ein Nachteil bei der Vermessung parallel der Nasallinie ist, dass man nicht rechtwinklig zum Luftweg misst und dadurch größere Strecken in anteriorer-posteriorer Richtung erhält. Zusätzlich ändert sich der Winkel zwischen der Nasallinie und der Körperachse je nach Kopflage. Um diesen Effekt zu minimieren, wurde immer die gleiche Kopfschale für die Aufnahme verwendet.

Durch die zunehmende Erfahrung des Untersuchers im Laufe der Auswertung der DVTDatensätze können systemische Fehler entstehen (Houston 1983). Erst nach entsprechender Einübung und Probeauswertungen erfolgte die Vermessung der DVT-Daten durch eine 
Person. Dadurch konnte die interindividuelle Fehlervariabilität eliminiert werden und evtl. Messfehler wurden als gleichbleibend angesehen.

Die Reproduzierbarkeit wurde beeinflusst durch Fehler bei der Lokalisation der zu vermessenden anatomischen Strukturen im DVT-Datensatz. Die nicht eindeutige Identifizierung einzelner anatomischer Referenzpunkte sei somit die häufigste Ursache für einen willkürlichen Messfehler (Houston 1983).

Aufgrund des hohen Kontrastes zwischen Hart- und Weichgeweben, bzw. Luft und Weichgeweben, ließen sich knöcherne Messpunkte und Messpunkte am Übergang von Luft zum Weichgewebe sehr gut identifizieren. Durch den geringen Kontrastunterschied der Weichgewebe untereinander konnten diese nicht unterschieden werden, was ein identifizieren der Messpunkte unmöglich machte. Dies trat auf, wenn die Zunge Kontakt zum weichen Gaumen hatte. Dann war die Bestimmung der Breite des weichen Gaumens nicht möglich (Abb. 30, 31 und 33). Auch bei anliegender Epiglottis an der Zunge war eine exakte Bestimmung der Epiglottisspitze und der Vallecula nicht möglich (Abb. 33).

Zusammenfassend ist anzumerken, dass durch die Tatsache, dass alle Datensätze an einer Referenzebene ausgerichtet und nur von einem Betrachter ausgemessen werden, die die gute Identifizierung der nötigen Referenzpunkte zu einer hohen Reproduzierbarkeit der Ergebnisse führte.

Um eine Aussage über die Dimension des Luftweges zu ermöglichen, verwendete diese Studie die sagittalen und transversalen Strecken zur Berechnung der Flächen. Der Luftweg wurde als Ellipse angenommen und mit der entsprechenden Formel berechnet. Diese Methode liefert aber nur einen Nährungswert. Koren et al. (2009), Fajdiga et al. (2005), Pepin et al. (1992) und Bohlman et al. (1983) haben mittels CT und Enciso et al. (2010) mittels DVT die Flächen des Luftweges mit entsprechenden Programmen exakt messen können.

Aufgrund der Möglichkeit der axialen Schnitte, konnte im Gegensatz zum Fernröntgenseitenbild der Luftweg auf jeder Höhe des Pharynx parallel zur Nasallinie transversal vermessen werden. Dabei war die größte anteriore-posteriore und die transversale Ausdehnung zu erkennen und die Fläche des Luftweges war berechenbar. Weiterhin war in den Axialschnitten zu erkennen, ob ein Luftweg geteilt, hantelförmig, schlitzförmig oder oval ist (Abb. 35 und 36). Bei den Fernröntgenseitenbildern kann aufgrund der zweidimensionalen Bilder und der Überlagerung von Strukturen nur die anteriore-posteriore Ausdehnung des Luftweges gemessen werden. Hochban et al. (1994) beschränkten sich in ihrer Studie auf 
seitliche Fernröntgenaufnahmen, Frontalaufnahmen wurden nicht berücksichtigt, da laut Aussage der Autoren die Weichteilstrukturen des Pharynx kaum zu identifizieren seien.

Durch die axialen Schnitte war es möglich, den Winkel der aufsteigenden Unterkieferäste zur Mediosagittalen zu bestimmen. Dies ist im Fernröntgenseitenbild nicht möglich.

Durch die Möglichkeit koronale Schnitte im DVT zu erstellen, war die palatinale Höhe, sowie der Abstand der lingualen Höcker zu messen. Johal und Conaghan (2004) mussten für die Bestimmung der palatinalen Höhe und für die Bestimmung des Abstandes zwischen den lingualen Höckern im Oberkiefer Modelle mittels Abdrücken herstellen und vermessen, da diese Informationen nicht aus dem Fernröntgenseitenbild zu ermitteln waren.

Zusätzlich ließ sich in den axialen Schnitten besser beurteilen, ob die Funktionskompartimente B (palatolinguales Kompartiment) und C (mesopharyngeales Kompartiment) voneinander getrennt waren oder seitlich der Mediosagittalen in Verbindung standen (Abb. 34). Beurteilt man so wie Koren et al. (2009) nur in einem mediosagittalen Schnitt, ob der weiche Gaumen der Zunge anliegt und betrachtet man nicht die gesamte mögliche Anlagefläche, sind Fehlinterpretationen hinsichtlich des Funktionszustandes des orofazialen Systems möglich. Auch in dem Fernröntgenseitenbild ist es nicht möglich, kleinere Verbindungen der Funktionskompartimente B und $\mathrm{C}$ zu diagnostizieren.

\subsection{Bedeutung der Messwerte als klinisch relevante Prognoseparameter}

\subsubsection{Korrelation von Messparametern und OSAS bzw. Schnarchen}

Abweichende Ergebnisse zu anderen Studien können dadurch zustande kommen, dass die Messbezugspunkte der einzelnen Studien zum Teil differieren, die Zusammensetzung der Patientenkollektive variiert, unterschiedliche Bildsequenzen und Schichtdicken beurteilt werden und teilweise die Aufnahmeposition zwischen den Studien differiert.

Aufgrund vorheriger Studien kann man bei OSAS-Patienten und Schnarchern einen dickeren und längeren weichen Gaumen, einen engeren Oropharynx und Isthmus, ein nach kaudal verlagertes Hyoid und ein verlängertes kollapsfähiges Segment erwarten. Der Hypopharynx sollte keinen Einfluss auf das OSAS oder Schnarchen haben. 
Auch in dieser Studie korrelierte die Länge des weichen Gaumens positiv mit der Lautstärke $(p<0,001)$ und Häufigkeit $(p<0,001)$ des Scharchens und dem Alter $(p=0,003)$ der Patienten. Dies zeigt, dass durch die Zunahme der Gaumenlänge im Alter, sich die Wahrscheinlichkeit für häufigeres und lauteres Schnarchen erhöht. Die Länge des weichen Gaumens hatte im Gegensatz dazu keinen signifikanten Einfluss auf den ESS-Wert, obwohl der Mittelwert bei den OSAS-bestätigten Patienten über $3 \mathrm{~mm}$ über dem Mittelwert des Gesamtkollektives lag. Somit kann man doch von einem Einfluss der weichen Gaumenlänge auf das OSAS nicht ausschließen.

Im Gegensatz zu anderen Studien hatte die Dicke des weichen Gaumens keinen signifikanten Einfluss auf das Schnarchen und den ESS-Wert. Dabei muss aber berücksichtigt werden, dass in dieser Studie nur bei etwas mehr als der Hälfte der Probanden die Dicke des weichen Gaumens gemessen werden konnte und somit die Aussagekraft dieses Parameters kritisch betrachtet werden muss.

Hochban et al. (1994) stellten bei OSAS-Patienten einen signifikant längeren und dickeren Gaumen fest, merkten aber an, dass die Verlängerung mit durchschnittlich $3 \mathrm{~mm}$, ebenso wie die Verdickung mit durchschnittlich unter $1 \mathrm{~mm}$ eher gering war. Tangugsorn et al. (1995) maßen bei OSAS-Patienten einen 19\% längeren und 17\% dickeren weichen Gaumen im Vergleich zur Kontrollgruppe. Auch Riley et al. (1983) ermittelten bei OSAS-Patienten einen signifikant längeren Gaumen (44 mm) im Gegensatz zur Kontrollgruppe (37 mm). Hillman et al. (2003) stellten einen vergrößerten weichen Gaumen bei OSAS-Patienten fest. Johal et al. (2007) wiesen daraufhin, dass der weiche Gaumen, so wie in dieser Studie auch, mit dem Alter länger wird, deshalb wählten sie eine altersangepasste Kontrollgruppe. Trotzdem war der weiche Gaumen bei OSAS-Patienten länger. Pepin et al. (1992) stellten fest, dass der weiche Gaumen bei OSAS-Patienten länger ist und sich in 6 von 10 Fällen an die dorsale Pharynxwand anlegt und so zum Verschluss führt und dabei in den Hypopharynx gesaugt wird.

Im Gegensatz dazu stellten Battagel et al. (2000) bei OSAS-Patienten und Schnarchern einen kürzeren und dickeren weichen Gaumen als bei der Kontrollgruppe fest. Dabei hatten die OSAS-Patienten aber einen signifikant dickeren weichen Gaumen als die Schnarcher. Battagel und L'Estrange (1996) konnten keinen Unterschied bei der Länge des weichen Gaumens zwischen OSAS-Patienten und Kontrollgruppe feststellen. Ebenso konnten Cuccia et al. (2007) bei der Zusammenfassung von 5 Studien keinen eindeutigen Zusammenhang zwischen OSAS-Patienten und der Länge des weichen Gaumens ermitteln. 
Der obere Luftweg wurde im Naso-, Oro- und Hypopharynx vermessen.

Die Ausmaße des Nasopharynx wurden auf Höhe der Nasallinie (UPW) vermessen und haben in dieser Studie keine signifikanten Korrelationen mit dem ESS-Wert und dem Schnarchen ergeben. Der Luftweg wird auf dieser Ebene durch knöcherne Strukturen vorgegeben und gestützt, dadurch kann es nicht zu einer Obstruktion kommen. Andererseits könnte ein verkleinertes, bzw. verlegtes Lumen den Luftwiderstand erhöhen und so die Obstruktionsneigung im Hypo- und Mesopharynx erhöhen. Ein erhöhter Luftwiderstand auf Höhe des harten Gaumens könnte aber auch die Mundatmung und somit das primäre Schnarchen fördern. Auch Oosterkamp et al. (2007) konnten auf dieser Ebene im Fernröntgenseitenbild keinen signifikanten Zusammenhang mit dem OSAS ermitteln. Dagegen konnten dort Tangugsorn et al. (1995) und Hochban et al. (1994) eine signifikante anterior-posteriore Einengung bei OSAS-Patienten nachweisen. Caballero et al. (1998) stellten in einer CT-Studie bei OSAS-Patienten eine signifikant kleinere Fläche hinter dem harten Gaumen fest.

Besondere Betrachtung wurde in dieser Studie auf den Oropharynx gelegt. Dieser wurde auf Höhe vom Isthmus (patientenabhängig) und auf Höhe der unteren Inzisivi (MPW) (standardisiert) vermessen.

Die Ausdehnung des Luftweges in der Mediosagittalen auf Höhe Isthmus korrelierte negativ mit dem ESS-Wert $(p=0,030)$, mit der Lautstärke $(p=0,001)$ und der Häufigkeit $(p=0,001)$ des Schnarchens und dem Alter $(\mathrm{p}=0,029)$ der Patienten. Mit dem BMI lag keine signifikante Korrelation vor. Die transversale Ausdehnung zeigte keine Zusammenhänge mit dem ESSWert und der Lautstärke des Schnarchens, aber eine negative Korrelation mit der Häufigkeit $(\mathrm{p}=0,005)$. Der Einfluss der mediosagittalen Ausdehnung reichte aber aus, dass die Luftwegsfläche negativ mit der Lautstärke $(p=0,005)$ und der Häufigkeit $(p<0,001)$ des Schnarchens korrelierten.

Auch Tangugsorn et al. (1995) und Hochban et al. (1994) konnten im Fernröntgenseitenbild eine signifikante Einengung anterior-posterior auf Höhe vom Isthmus bei OSAS-Patienten nachweisen. Solow et al. (1996) fanden mittels Fernröngtenaufnahmen an dieser Stelle des Luftweges den größten Unterschied zwischen OSAS- und Kontrollgruppe (bei OSASPatienten fast 50\% enger; 10,1 mm gegenüber 5,2 mm). Johal et al. (2007) ermittelten mit Hilfe des Fernröntgenseitenbildes, dass der Luftweg zwischen weichem Gaumen und dorsaler Pharynxwand bei OSAS-Patienten im Durchschnitt nur 2/3 der Kontrollgruppe betrug. 
Im Gegensatz zu dieser Studie fanden Enciso et al. (2010) mittels DVT bei OSAS-Patienten anterior-posterior keine signifikante Verengung auf Höhe Isthmus. Übereinstimmend mit dieser Studie gaben sie an, dass die Fläche des Luftweges am Isthmus bei OSAS-Patienten signifikant kleiner ist. Die kleinere Fläche käme aber vor allem durch die signifikant kleinere laterale Ausdehnung zu Stande. Enciso et al. (2010) kamen zu dem Schluss, wenn die laterale Dimension kleiner als $17 \mathrm{~mm}$ sei, bestehe ein erhöhtes Risiko für das OSAS. Auch Fajdiga (2005) konnte im CT transversal die engste Stelle hinter dem weichen Gaumen lokalisieren, kranial und kaudal erweiterte sich der Luftweg wieder. Er konnte aber in allen Ebenen transversal keinen signifikanten Unterschied zwischen Schnarchern und Nichtschnarchern feststellen.

Avrahami und Englender (1995) konnten im CT bei OSAS-Patienten eine kleinere Luftwegsfläche auf Höhe Isthmus nachweisen und schlossen daraus, dass dies eine bedeutende Rolle für die Entwicklung vom OSAS haben könnte. Auch Vos et al. (2007) betonten nochmals die Bedeutung der minimalen Fläche des Luftweges, sie korreliere mit dem AHI negativ.

Die Lage des Isthmus zur Nasalline hatte einen Einfluss auf die Lautstärke $(p=0,006)$ des Schnarchen, aber nicht auf dessen Häufigkeit. Je größer der Abstand ist, desto mehr kann der weiche Gaumen schwingen und die Lautstärke steigt an.

Die Strecke des Luftweges auf Höhe der unteren Inzisivi (MPW) in sagittaler Richtung korrelierte negativ mit der Lautstärke $(p=0,005)$ und der Häufigkeit $(p=0,042)$ des Schnarchens und dem Alter $(\mathrm{p}=0,002)$ der Patienten. Die transversale Ausdehnung auf dieser Höhe des Luftweges hat keine signifikanten Einflüsse ergeben. Doch war der sagittale Einfluss so groß, dass die Fläche MPW negativ mit der Lautstärke ( $\mathrm{p}=0,002)$ und Häufigkeit $(p=0,011)$ des Schnarchens korrelierte. Mit dem ESS-Wert ergab sich auf dieser Ebene des Luftweges kein signifikanter Einfluss. Auch andere Studien konnten anhand von Fernröntgenseitenbildern bei OSAS-Patienten einen signifikant kleineren Oropharynx in anteriorer-posteriorer Richtung nachweisen (Osoterkamp et al. 2007, Battagel et al. 2000, Battagel und L'Estrange 1996, Hochban et al. 1994). Partinen et al. (1988) kamen zu dem Schluss, dass, wenn der Luftweg auf Höhe der Mandibularlinie sagittal kleiner als $5 \mathrm{~mm}$ sei, dieser positiv mit dem AHI-Wert korreliere. Eine reduzierte Fläche des Luftweges auf Höhe des Oropharynx konnten auch Caballero et al. (1998) und Pepin et al. (1992) mittels CT bei OSAS-Patienten nachweisen. 
Der Hypopharynx wurde auf Höhe des dorsalsten Punktes der Zunge (TPW) und der Vallecula (LPW) vermessen.

Die Dimension des Luftweges auf Höhe des dorsalsten Punktes der Zunge (TPW) hatte keine signifikanten Auswirkungen auf den ESS-Wert, auf die Lautstärke und Häufigkeit des Schnarchens. Auch Battagel und L’Estrange (1996) konnten keinen Unterschied zwischen OSAS-Patienten und Kontrollgruppe in der Luftwegsdimension hinter der Zunge feststellen. Im Gegensatz dazu stellten Battagel et al. (2000) im Fernröntgenseitenbild einen signifikant kleineren Luftweg bei OSAS-Patienten und Schnarchern im Vergleich zur Kontrollgruppe fest. Pepin et al. (1992) wiesen auf die Bedeutung der Zunge bei der Obstruktion der Atemwege hin. Bei allen, von ihnen untersuchten Patienten bewegte sich die Zunge bei der Apnoe rückwärts in den Oropharynx. Hora et al. (2007) konnten einen Zusammenhang zwischen dem transversalen Durchmesser retroglossal und dem OSAS nachweisen. Bei einem kleineren Durchmesser als $12 \mathrm{~mm}$ liege eine hohe Wahrscheinlichkeit für eine schwere Form vom OSAS vor.

Die Luftwegsdimensionen auf Höhe der Vallecula (LPW) hatten keinen Einfluss auf den ESS-Wert, die Lautstärke und die Häufigkeit des Schnarchens. Auf dieser Höhe des Luftweges schien es nur geringe anatomische Unterschiede zwischen den Probanden zu geben (geringe Standardabweichungen bei den Mittelwerten). Der Luftweg war dort größer als weiter kranial und des Weiteren stabilisieren die knorpeligen Strukturen des Larynx den Luftweg. Auch andere Autoren konnten keine Unterschiede in sagittaler Richtung feststellen (Tangugsorn et al. 1995, Hochban et al. 1994), doch Solow et al. (1996) konnten auf dieser Ebene bei OSAS-Patienten eine sagittale Einengung im Fernröntgenseitenbild ermitteln. Caballero et al. (1998) konnten in einer CT-Studie nur bei der Expiration eine signifikant vergrößerte Hypopharynxfläche bei OSAS-Patienten nachweisen.

Insgesamt erschien bei der Betrachtung der Korrelationen die transversale Ausdehnung des Luftweges in dieser Studie keine signifikanten Auswirkungen auf das primäre Schnarchen und auf den ESS-Wert zu haben. Doch bei der Beachtung der Mittelwerte zeigte sich bei den Patienten mit bestätigtem OSAS auf allen gemessenen Ebenen ein kleinerer transversaler Durchmesser des Luftweges als im Vergleich zum Gesamtkollektiv.

Viele vorherigen Studien wurden anhand von Fernröntgenseitenbildern durchgeführt und somit die transversale Dimension des Luftweges nicht betrachtet. Doch andere Autoren, die den Luftweg dreidimensional darstellten, sprechen der transversalen Komponente besondere 
Bedeutung beim OSAS zu. So fanden Schwab et al. (1995) bei OSAS-Patienten vorwiegend laterale Luftwegseinengungen, aber keine signifikanten Unterschiede in der sagittalen Ausdehnung. Ebenso konnten Li et al. (2002) keinen Unterschied in der anteriorenposterioren Luftwegsdimension feststellen. Auch Enciso et al. (2010) schließen aus ihren Ergebnissen, dass die Präsenz und Schwere vom OSAS mit einer lateralen Einengung des Luftweges assoziiert sei. Weiterhin lassen die Ergebnisse von Fairburn et al. (2007) auf eine zentrale Rolle der transversalen Dimension des Luftweges schließen. Sie bemerkten, dass bei einer operativen OK- und UK-Vorverlagerung der laterale Durchmesser relativ stärker als der anteriore-posteriore Durchmesser erweitert wird. Nur auf Höhe des Hyoids konnte keine laterale Erweiterung festgestellt werden. Durch dieses operative Vorgehen verbesserte sich bei 18 von 20 Patienten der AHI.

Die Strecke PNS-Epiglottis korrelierte in dieser Studie positiv mit der Lautstärke ( $\mathrm{p}=0,001)$ und mit der Häufigkeit $(\mathrm{p}=0,014)$ des Schnarchens, hatte aber keine signifikanten Auswirkungen auf den ESS-Wert. Die Korrelationen gaben einen Hinweis auf eine erhöhte Wahrscheinlichkeit für primäres Schnarchen, aber keinen Hinweis auf das OSAS. Die Strecke PNS-Epiglottis gibt die Länge des kollapsfähigen Weichteilschlauches zwischen dem harten Gaumen und der knorpeligen Epiglottisspitze an. Mit zunehmender Länge verliert der Weichgewebsschlauch an Stabilität, weshalb neben der positiven Korrelation mit dem primären Schnarchen eigentlich auch eine positive Korrelation mit dem ESS-Wert zu erwarten gewesen wäre. Wenn man aber die Mittelwerte betrachtet, stellt man fest, dass bei den OSAS-bestätigten Patienten die Strecke um fast $7 \mathrm{~mm}$ länger als bei dem Gesamtkollektiv war. Somit lässt sich bei OSAS-Patienten eine tendenziell längere Strecke bestätigen.

Auch Schäfer (1996) ging bei OSAS-Patienten von einem verlängerten kollapsfähigen Segment aus. Dagegen konnten Johal et al. (2007) zwischen OSAS-Patienten und Kontrollgruppe keine Unterschiede in der Pharynxhöhe (Nasallinie zu Epiglottisspitze) feststellen. Eine leicht reduzierte Pharynxhöhe konnten dagegen Battagel et al. (2000) bei OSAS-Patienten gegenüber der Kontrollgruppe nachweisen. Die Abweichungen können ihre Ursache durch eine leicht veränderte Messstrecke oder an der Kollektivzusammensetzung haben.

Die Lage des Hyoids schien einen Einfluss auf die Prävalenz vom OSAS und primären Schnarchen zu haben. Die Strecke Hyoid-Nasallinie korrelierte positiv mit der Lautstärke 
( $\mathrm{p}=0,034)$ und schwach mit der Häufigkeit $(\mathrm{p}=0,049)$ des Schnarchens und dem Alter $(\mathrm{p}=0,008)$. Im Gegensatz dazu, korrelierte die Stecke negativ mit dem ESS-Wert $(\mathrm{p}=0,020)$. Eine vergrößerte Strecke könnte auch auf ein verlängertes kollapsfähiges Segment hinweisen. Die knöcherne Struktur des Hyoids dient der hyoidalen Muskelatur als Ansatz und stabilisiert somit auch die Luftwegsdimensionen. Bei zunehmendem Alter scheint sich die Strecke zu verlängern, wodurch die stabilisierende Wirkung nachlässt. Auch Oosterkamp et al. (2007) konnten einen signifikanten Einfluss des Alters auf die Hyoid-Position ermitteln.

Johal et al. (2007) konnten bei OSAS-Patienten einen größeren Abstand des Hyoids zur Nasallinie messen und stellten die These auf, dass durch die tiefere Lage des Hyoids die Zunge weiter nach hinten gezogen werde und dadurch den Pharynx einengen könnte.

Dagegen konnten Battagel et al. (2000) keinen signifikanten Unterschied zwischen OSASPatienten und Kontrollgruppe bezüglich der Strecke Hyoid-Nasallinie feststellen, aber einen vergrößerten Abstand des Hyoids zur Mandibularline und ein weiter posterior liegendes Hyoid bei OSAS-Patienten. Hochban et al. (1994) bestätigten die weiter dorso-kaudale Lage des Hyoids, wiesen jedoch darauf hin, dass sich im Einzelfall die Zungenbeinposition als sehr variabel, mit großer Schwankungsbreite, erwies.

In der vorliegenden Studie konnten keine signifikanten Zusammenhänge bezüglich der sagittalen Lage des Hyoids (ANS-Hyoidsenkrechten) festgestellt werden. Dies könnte neben unterschiedlichen Kollektiven auch an unterschiedlichen Referenzpunkten zur Lagebestimmung des Hyoids liegen.

Der Abstand des Hyoids zur Mandibularlinie scheint einen größeren Einfluss auf das OSAS zu haben als die Lage zur Nasallinie. Mehrere Studien konnten eine erhöhte Distanz zwischen dem Hyoid und der Mandibularlinie bei OSAS-Patienten bestätigen (Oosterkamp et al. 2007, Riha et al. 2005, Hillman et al. 2003, Hochban et al. 1994, Partinen et al. 1988, Riley et al. 1983). Riley et al. (1983) gaben den durchschnittlichen Hyoid-Abstand zur Mandibularlinie mit $29 \mathrm{~mm}$ für OSAS-Patienten und mit $16 \mathrm{~mm}$ für die Kontrollgruppe an. Partinen et al. (1988) wiesen eine positive Korrelation mit dem AHI-Wert nach, wenn der Abstand Hyoid zur Mandibularline größer als 24 mm war. Laut Riha et al. (2005) könnte eine vergrößerte Distanz des Hyoids zur Mandibularlinie ein Indikator für die Schwere von OSAS sein. Bei der Zusammenfassung von 5 Studie kamen Cuccia et al. (2007) zu dem Ergebnis, dass sich das Hyoid bei OSAS-Patienten kaudaler befindet.

Die Aussage über die negative Korrelation von dem ESS-Wert mit der Strecke HyoidNasallinie hielt bei der Plausibilitätskontrolle nicht stand. Bei der Betrachtung der Mittelwerte zeigte sich eine gegenläufige Tendenz. Patienten mit bestätigtem OSAS hatten im Mittel eine 
um $1 \mathrm{~cm}$ größere Strecke. Allerdings konnte der Mittelwert für die OSAS-bestätigten Patienten nur anhand von 3 Patienten ermittelt werden. Bei den anderen OSAS-Patienten lag das Hyoid außerhalb des Scanbereiches und konnte somit nicht in der Routineuntersuchung erfasst werden, daher kann man von noch größeren Werten ausgehen.

Diese Studie konnte keinen Zusammenhang zwischen der Strecke ANS-PNS mit dem ESSWert, der Häufigkeit des Schnarchens und dem Alter nachweisen, im Gegensatz dazu, bestand ein geringer Zusammenhang mit der Lautstärke des Schnarchens ( $\mathrm{p}=0,045)$. Ein größeres Mittelgesicht weist wahrscheinlich auch einen längeren weichen Gaumen auf, der die Zunahme der Lautstärke des Schnarchens erklären kann.

Auch Oosterkamp et al. (2007) fanden für diese Strecke keinen signifikanten Unterschied zwischen der OSAS- und Kontrollgruppe. Andererseits beschrieben Johal et al. (2007) einen signifikant kürzeren harten Gaumen bei OSAS-Patienten, wobei die Differenz zur Kontrollgruppe nur 1,3 mm betrug. Der dabei angegebene Durchschnittswert für OSASPatienten (47,0 mm \pm 3,4 Stdabw.) liegt deutlich unter dem Mittelwert dieser Studie. Die unterschiedlichen Werte können mit einer anderen Zusammensetzung des Kollektives erklärt werden.

In dieser Studie konnten keine Zusammenhänge vom OSAS oder primären Schnarchen mit der Unterkieferlänge nachgewiesen werden. Auch Bacon et al. (1990) konnten keine reduzierte Unterkieferlänge bei OSAS-Patienten nachweisen. Andere Autoren dagegen konnten eine signifikante Reduktion der Unterkieferlänge nachweisen (Johal et al. 2007, Hillman et al. 2003, Battagel und L'Estrange 1996). Battagel et al. (2000) konnten keinen Unterschied zwischen OSAS-Patienten und Schnarchern bezüglich der Unterkieferlänge feststellen, aber bei Kontrollgruppe war die Strecke fast $2 \mathrm{~mm}$ länger.

Battagel und L'Estrange (1996) konnten zusätzlich eine verminderte intermaxilläre Raumlänge bei OSAS-Patienten nachweisen, woraus sie schlossen, dass der Funktionsraum der Zunge eingeschränkt werde. Auch Johal et al. (2007) konnten eine um 3,1 mm kürzere intermaxilläre Raumlänge bei OSAS-Patienten nachweisen und merkten an, zusammen mit einem verkürzten Unterkiefer stehe weniger Funktionsraum für die Zunge zur Verfügung und damit steige das Risiko für das OSAS. Bestätigen konnten Battagel et al. (2000), dass bei OSAS-Patienten und Schnarchern die intermaxilläre Raumlänge im Gegensatz zur Kontrollgruppe um ca. $5 \mathrm{~mm}$ auf $74 \mathrm{~mm}$ reduziert ist. 
In der vorliegenden Studie konnten keine Zusammenhänge zwischen der intermaxillären Raumlänge und dem OSAS, bzw. dem Schnarchen aufgezeigt werden.

Die Messergebnisse aus dem koronalen Schnitt ergaben keine signifikanten Hinweise auf das OSAS oder primäres Schnarchen.

Bei einem kleineren Außenwinkel des aufsteigenden Unterkieferastes auf Höhe der unteren Inzisivi stieg die Wahrscheinlichkeit für lauteres $(p<0,001)$ und häufigeres $(p<0,001)$ Schnarchen an. Des Weiteren korrelierte der Außenwinkel negativ mit dem BMI ( $p=0,040)$, es bestand aber keine signifikante Korrelation zum ESS-Wert. Die Neigung des aufsteigenden Unterkieferastes relativ zur Frontalebene scheint Einfluss auf die Stabilität der Weichgewebe des oberen Luftweges zu haben. Es kann vermutet werde, dass beim Tonusverlust während des Schlafes der außen ansetzende Muskel (M. masseter) bei kleinerem Außenwinkel nicht das Zurückfallen des UK verhindern kann. Pepin et al. (1992) konnten am Ende der Apnoe eine Unterkieferbewegung nachweisen. Auch Hollowell und Suratt (1991) stellten fest, dass der M. masseter am Ende der Apnoe aktiviert wird.

Beim erhöhten BMI verringerte sich der Außenwinkel $(\mathrm{p}=0,040)$, da sich auch die transversale Breite des Unterkieferkörpers (Abstand lingualer Höcker) vergrößerte $(p=0,011)$. Dabei änderte sich aber der Innenwinkel nicht signifikant.

Der Innenwinkel hatte keinen signifikanten Einfluss auf das Schnarchen oder auf das OSAS, obwohl zu erwarten wäre, dass der Innenwinkel einen größeren Einfluss auf die Luftwegsdimension haben müsste. Die Vermessung der Winkel erfolgte mittels einer eigens entwickelten Methode, weshalb ein Vergleich mit anderen Untersuchungen nicht möglich war.

Im untersuchten Patientengut schien das Alter und das Gewicht eine vernachlässigbare Rolle für den ESS-Wert zu haben. In dieser Studie ergaben sich keine signifikanten Korrelationen zwischen dem ESS-Wert und dem Alter, bzw. dem BMI. Doch bei der Betrachtung der Mittelwerte wurde deutlich, dass Patienten mit bestätigtem OSAS im Vergleich zum Gesamtkollektiv ein höheres Durchschnittssalter und einen höheren BMI aufweisen. Wiederrum nahmen Lautstärke und Häufigkeit des Schnarchens mit zunehmendem Alter und BMI der Patienten signifikant zu.

In anderen Studien wiesen die OSAS-Patienten einen signifikant höheren BMI auf (Hora et al. 2007, Johal et al. 2007, Pracharktam et al. 1996). Auch konnte eine positive Korrelation 
zwischen BMI und AHI nachgewiesen werden (Vos et al. 2007), zudem soll der BMI bei OSAS-Patienten signifikant größer sein als bei Schnarchern (Enciso et al. 2010). Laut Kim et al. (2010) bewirkt ein erhöhter BMI eher eine erhöhte Kollapsfähigkeit als ein verminderter Durchmesser des oberen Luftweges.

Ein erhöhter BMI korrelierte in dieser Studie mit einer transversalen Einengung des Luftweges auf allen gemessenen Ebenen (UPW ( $p<0,001)$, MPW ( $<<0,001)$, Isthmus $(p<0,001)$, TPW ( $p=0,032)$, LPW ( $p=0,032)$ ), es ergab sich aber kein Zusammenhang mit der anteriorenposterioren Dimension. Außer bei der Fläche auf Höhe der Vallecula (TPW) reichte die transversale Komponente aus, um einen statistischen Einfluss auf die Fläche zu bewirken. Dabei nahm die Fläche des Luftweges mit steigendem BMI ab.

Partinen et al. (1988) stellten fest, dass bei niedrigem BMI anatomische Besonderheiten größeren Einfluss auf den AHI-Wert haben, mit steigendem BMI nimmt deren Einfluss ab und der BMI wird wichtiger. Horner et al. (1989) berichteten über Fettdepots in der lateralen Pharynxwand bei übergewichtigen Patienten, die bei OSAS-Patienten signifikant vergrößert waren. Zusätzlich wiesen nur die OSAS-Patienten auch Fettdepots im weichen Gaumen auf. Schwab et al. (1995) stellten dagegen mittels MRT fest, die Dicke der lateralen Pharynxmuskulatur habe größeren Einfluss auf die anatomische Verengung als die Vergrößerung der Fettdepots. Auch andere Autoren konnten keinen signifikanten Zusammenhang zwischen parapharyngealer Fettzunahme und OSAS nachweisen, aber eine tendenziell dickere parapharyngeale Muskulatur bestätigen (Hora et al. 2007). Li et al. (2002) konnten eine Zunahme der Dicke der lateralen Pharynxwand bei OSAS-Patienten bestätigen.

Caballero et al. (1998) konnten in einer CT-Studie nachweisen, dass das retropharyngeale Gewebe bei OSAS-Patienten und Patienten mit erhöhtem BMI signifikant dicker sei. Der Person-Korrelations-Koeffizient lag bei der Dicke des retropharyngealen Gewebes und dem BMI bei 0,63, wobei die Autoren die pharyngealen Gewebearten nicht weiter differenzierten.

Des Weiteren zeigte diese Studie weitere anatomische Veränderungen, die mit dem BMI korrelieren. Je größer der BMI war, desto größer wurde der Abstand der Pharynxhinterwand zu dem unteren Inzisalpunkt (intermaxilläre Raumlänge) ( $p<0,039)$. Auch in dem koronalen Schnitt zeigten sich skelettale Unterschiede, die mit dem BMI korrelierten. So nahm mit steigendem BMI die Höhe der Mundhöhle (Strecke Nasallinie-Unterkiefer) $(p<0,048)$ und die Breite der Mundhöhle (Abstand der lingualen Höcker) ( $\mathrm{p}<0,011)$ zu und der Außenwinkel des aufsteigenden Unterkieferastes nahm ab ( $\mathrm{p}<0,040)$. Diese Unterschiede resultierten durch die vergrößerte Anatomie des Unterkieferkörpers, die palatinale Höhe des Oberkiefers wies keine signifikante Korrelation mit dem BMI auf. 


\subsubsection{Orofazialer Systemzustand und OSAS}

Der Mann-Whitney-U-Test zeigte einen signifikanten Unterschied zwischen dem Systemzustand offen und geschlossen bezüglich der Kontaktstrecke zwischen weichem Gaumen und Zunge $(p<0,001)$, der Lufthöhe unter dem harten Gaumen $(p<0,001)$, der Strecken MPW transversal $(p=0,019)$ und Isthmus transversal $(p=0,035)$ und der Fläche auf Höhe Isthmus ( $\mathrm{p}=0,016)$.

Wie zu erwarten, erkennt man in den Box-Plots, dass bei einem offenen Systemzustand die Kontaktstrecke der Zunge mit dem weichen Gaumen tendenziell geringer war und dass die Lufthöhe unter dem weichen Gaumen tendenziell größer war. Die kürzere Anlagestrecke der Zunge kann dadurch erklärt werden, dass im offenen Systemzustand die Zunge nicht oder nur teilweise am weichen Gaumen anlag, das heißt, die Funktionskompartimente B (palatolinguales Kompartiment) und C (mesopharyngeales Kompartiment) standen in Verbindung. Während des offenen Systemzustandes fehlte im Funktionskompartimente B der Unterdruck, der ein Anlegen der Zunge an den harten Gaumen förderte und so resultierte eine größere Lufthöhe unter dem harten Gaumen.

Die Box-Plots für die Strecke MPW transversal, Isthmus transversal und für die Fläche des Luftweges auf Höhe Isthmus zeigten bei einem offenen Systemzustand eine tendenziell größere Ausdehnung. Dabei waren die metrischen Unterschiede zum geschlossenen Systemzustand sehr gering, sodass die Aussagekraft dieser Zusammenhänge als sehr eingeschränkt zu betrachten ist. Die Gemeinsamkeiten der Messungen auf Höhe von MPW und Isthmus können durch die nahe Lagebeziehung der beiden Messebenen zueinander erklärt werden.

Bei dem offenen Systemzustand hätte man eine sagittale Einengung des Luftweges im Bereich des Oropharynx erwartet, da der weiche Gaumen nicht an der Zunge anliegt und durch die Schwerkraft nach dorsal verlagert wird und somit den Luftweg einengt.

Die ermittelten Zusammenhänge könnten entweder durch eine transversale Kompensation des offenen Systemzustandes durch einen erhöhten Muskeltonus im Wachzustand erklärt werden oder der breitere, bzw. größere Luftweg begünstigt einen offenen Systemzustand.

Doch konnten in dieser Studie keine signifikanten Unterschiede des Systemzustandes bezüglich der ESS-Werte, der Lautstärke oder der Häufigkeit des Schnarchens aufgezeigt werden. Als Fazit scheint der orofaziale Systemzustand während der DVT-Aufnahme nicht konstant zu sein. Offensichtlich scheinen andere Faktoren größeren Einfluss auf das OSAS und Rhonchopathienen zu haben, somit lässt dieser Parameter keine eindeutige Aussage zu. 


\subsection{Schlussfolgerung}

Durch die vermehrte Nutzung der dreidimensionalen Diagnostik in der zahnärztlichen Praxis wird die Verbreitung des DVT zunehmen. Besonders bei älteren Patienten wird zunehmend öfter die DVT angewendet und könnte so eher als ein Fernröntgenseitenbild für ein Screeningverfahren für Rhonchopathien und dem OSAS dienen.

Diese Studie hat gezeigt, dass mithilfe des DVT zusätzlich der Luftweg und angrenzende Strukturen ohne weitere Strahlenbelastung für den Patienten dargestellt und vermessen werden können und somit auch zum Bestandteil der diagnostischen Auswertung gehören sollte. Anhand eines Durchschnittskollektives konnten Zusammenhänge zwischen der Anatomie des orofazialen Systems und dem Auftreten von Rhonchopathien und dem OSAS dargestellt werden. Die DVT stellt nach den Ergebnissen dieser Studie ein nützliches Verfahren dar, um folgende Parameter als Indikatoren für Pathologien des oberen Luftweges, in diesem Fall die Länge des weichen Gaumens, die Länge des kollapsfähigen Segmentes, die Strecke Hyoid zur Nasallinie, der Außenwinkel, sowie die sagittale Dimension und Fläche des Luftweges auf Höhe vom Isthmus, hinsichtlich einer möglichen Rhonchopathie oder des OSAS erfolgreich zu bestimmen.

Mit diesen Erkenntnissen ist es auch dem Zahnarzt möglich, als Nebenbefund ein Risiko für Rhonchopathien frühzeitig zu diagnostizieren. Daher sollten die oben genannten Punkte bei jeder DVT-Auswertung routinemäßig berücksichtigt werden. Bei kritischen Werten sollte der Patient in ein Schlaflabor zur weiteren Abklärung überwiesen werden. Diese Risikobewertung hat eine vitale Relevanz für den Patienten.

In weiteren Studien sollte das Patientenkollektiv mit Hilfe der Polysomnographie in eine Kontroll- und in eine OSAS-Gruppe unterteilt werden, um die Nutzbarkeit des DVT im Rahmen der Rhonchopathie- und OSAS-Diagnostik besser beurteilen zu können. Zur Qualitätsverbesserung und Standardisierung der DVT-Aufnahme wäre es sinnvoll, ein Zungenrepositionsmanöver unter Kontrolle einer druckanzeigenden Membrantrichterplatte durchzuführen.

Eine Ausweitung des Einsatzes des DVT bei der Diagnostik des OSAS könnte in Zukunft dazu dienen, die einzelnen Obstruktionsmechanismen über eine exakte anatomische Darstellung besser zu verstehen. Zusätzlich könnte der Einfluss von zahnärztlichen Geräten, z.B. mit eingesetzter Protrusionsschiene, kontrolliert werden. 


\section{Zusammenfassung}

Hintergrund: Mit dem digitalen Volumentomographen (DVT) ist es dem Zahnarzt seit 1997 möglich, in der Praxis eine dreidimensionale Bildgebung zu betreiben. Ziel dieser Untersuchung war es, die Möglichkeit des DVT für die Diagnostik des OSAS und Rhonchopathien $\mathrm{zu}$ überprüfen und Parameter für ein Screeningverfahren zur Risikobewertung zu benennen.

Methodik: Aus den ab 2006 bis Anfang 2009 vorhandenen DVT-Datensätzen, die unabhängig von einem OSAS-Verdacht erstellt wurden, wurden konsekutiv 104 Datensätze ausgewählt. Das Patientenkollektiv umfasste 104 Patienten im Alter von 30 bis 68 Jahren (52 Männer mit einen Durchschnittsalter von 46,82 Jahre \pm 11,25 Stdabw. und 52 Frauen mit einem Durchschnittsalter von 46,48 Jahre \pm 11,32 Stdabw.).

Die Patienten wurden im wachen und liegenden Zustand, mit geschlossenem Mund und in habitueller Interkuspidation untersucht. Einschlusskriterien waren vollständig dargestellter Bereich vom harten Gaumen bis zu Vallecula bzw. Hyoid und ausgefüllter Schlaffragebogen. Der Fragebogen umfasste Angaben zum Alter, zum Gewicht, zur Körpergröße und die Epworth Sleepiness Scale, sowie Daten zur Lautstärke und Häufigkeit des Schnarchens, bewertet auf einer visuellen Analogskala.

Die DVT-Aufnahmen wurden in der Universitätsklinik Göttingen, Abteilung Mund-, Kieferund Gesichtschirurgie mit dem digitalen Volumentomographen der Firma NewTom QR-9000 (Quantitative Radiology, Verona, Italien) erstellt. Der DVT arbeitet mit einer konstanten Spannung von $110 \mathrm{kV}$, der Röhrenstrom liegt je nach Knochendichte zwischen 0,5 und 10 mA. Die DVT-Auswertung wurde mit dem Softwareprogramm NewTom 9000 Dental Version 3.37 durchgeführt. Eine Signifikanz wurde bei p $<0,05$ angenommen.

Resultate: Es konnte eine positive Korrelation zwischen der Länge des weichen Gaumens mit der Lautstärke $(p<0,001)$ und Häufigkeit $(p<0,001)$ des Scharchens und mit dem Alter $(p=0,003)$ der Patienten festgestellt werden. Bei den Patienten mit bestätigtem OSAS lag der Mittelwert für die weiche Gaumenlänge $3 \mathrm{~mm}$ über dem des Gesamtkollektives.

Die Dimension des Luftweges auf Höhe des Naso- und Hypopharynx (TPW und LPW) hatte keine signifikanten Auswirkungen auf den ESS-Wert, sowie auf die Lautstärke und Häufigkeit des Schnarchens.

Im Gegensatz dazu korrelierte die sagittale Ausdehnung des Luftweges auf Höhe Isthmus negativ mit dem ESS-Wert $(\mathrm{p}=0,030)$, mit der Lautstärke $(\mathrm{p}=0,001)$ und mit der Häufigkeit $(\mathrm{p}=0,001)$ des Schnarchens und dem Alter $(\mathrm{p}=0,029)$ der Patienten. Ebenso korrelierte die 
Luftwegsfläche auf Höhe Isthmus negativ mit der Lautstärke ( $\mathrm{p}=0,005)$ und der Häufigkeit $(\mathrm{p}<0,001)$ des Schnarchens.

Die sagittale Strecke des Luftweges auf Höhe MPW korrelierte negativ mit der Lautstärke ( $p=0,005)$ und Häufigkeit $(\mathrm{p}=0,042)$ des Schnarchens und dem Alter $(\mathrm{p}=0,002)$ der Patienten, die Luftwegsfläche korrelierte negativ mit der Lautstärke ( $\mathrm{p}=0,002)$ und Häufigkeit ( $\mathrm{p}=0,011)$ des Schnarchens. Bei der Beachtung der Mittelwerte zeigte sich bei den Patienten mit bestätigtem OSAS auf allen gemessenen Ebenen ein kleinerer transversaler Durchmesser des Luftweges als im Vergleich zum Gesamtkollektiv.

Die Strecke des kollapsfähigen Weichteilschlauches (PNS-Epiglottis) korrelierte in dieser Studie positiv mit der Lautstärke $(\mathrm{p}=0,001)$ und mit der Häufigkeit $(\mathrm{p}=0,014)$ des Schnarchens, hatte aber keine signifikanten Auswirkungen auf den ESS-Wert, wobei bei den OSAS-bestätigten Patienten die mittelwertige Strecke um fast $7 \mathrm{~mm}$ länger als bei dem Gesamtkollektiv ist.

Die Strecke Hyoid-Nasallinie korrelierte positiv mit der Lautstärke $(\mathrm{p}=0,034)$ und schwach mit Häufigkeit $(\mathrm{p}=0,049)$ des Schnarchens und dem Alter $(\mathrm{p}=0,008)$, im Durchschnitt war die Strecke bei Patienten mit bestätigtem OSAS um $1 \mathrm{~cm}$ größer.

Im Gegensatz zum Außenwinkel des aufsteigenden Unterkieferastes, relativ zur Frontalebene gemessen, welcher negativ mit der Lautstärke $(p<0,001)$ und Häufigkeit $(p<0,001)$ des Schnarchens korrelierte, wies der Innenwinkel keine signifikante Korrelation auf.

In dieser Studie konnten keine Zusammenhänge vom OSAS oder von Rhonchopathien mit der Unterkieferlänge, der intermaxillären Raumlänge, den Messergebnissen aus dem koronalen Schnitt und dem Systemzustand des orofazialen Systems aufgezeigt werden.

Schlussfolgerung: Nach der vorliegenden Studie hat sich die Erhebung folgender relevanter Parameter für die schlafmedizinische Diagnostik ergeben: Länge des weichen Gaumens, Länge des kollapsfähigen Segmentes, Strecke Hyoid zur Nasallinie, Außenwinkel des aufsteigenden Unterkieferastes, sagittale Dimension und Fläche des Luftweges auf Höhe vom Isthmus. Diese Studie hat gezeigt, dass es mit dem DVT auch in der zahnärztlichen Praxis möglich ist, eine Risikobewertung für das OSAS und die Rhonchopathien zu erheben. Daher sollten die oben genannten Punkte bei der DVT-Auswertung routinemäßig berücksichtigt werden. 


\section{Abkürzungsverzeichnis}

\begin{tabular}{|c|c|}
\hline Abb.: & Abbildung \\
\hline AHI: & Apnoe-Hypopnoe-Index \\
\hline ANS: & Spina nasalis anterior \\
\hline ASDA: & American Sleep Disorders Association \\
\hline BMI: & Body-Mass-Index \\
\hline BV: & Bildverstärker \\
\hline CCD: & Charge-Couple-Device \\
\hline cm: & Zentimeter \\
\hline CT: & Computertomograph /-graphie /-gramm \\
\hline 3D: & dreidimensional \\
\hline DVT: & Digitaler Volumentomograph /-graphie /-gramm \\
\hline EEG: & Elektroenzephalographie \\
\hline EKG: & Elektokardiogramm \\
\hline EMG: & Elektromyogaphie \\
\hline EOG: & Elektrookulographie \\
\hline ESS: & Epworth Sleepiness Scale \\
\hline FRS: & Fernröntgenseitenbild \\
\hline HE: & Hounsfield-Einheiten \\
\hline Hz: & Hertz \\
\hline ICSD: & international classification of sleep disorders \\
\hline $\mathrm{kV}$ : & Kilovolt \\
\hline LPW: & Lower Pharyngeal Wall \\
\hline M.: & Musculus \\
\hline mA: & Milliampere \\
\hline Max.: & Maximum \\
\hline mGy: & Milligray \\
\hline Min.: & Minimum \\
\hline mm: & Millimeter \\
\hline $\mathrm{mm} \mathrm{H}_{2} \mathrm{O}$ : & Millimeter Wassersäule \\
\hline MPW: & Middle Pharyngeal Wall \\
\hline MRT: & Magnetresonanztomograph /-graphie /-gramm \\
\hline $\mathrm{mSv}:$ & Millisievert \\
\hline$\mu \mathrm{Sv}:$ & Mikrosievert \\
\hline n-CPAP: & nasal continuous positive airway pressure \\
\hline NL: & Nasallinie \\
\hline Non-REM: & non-rapid-eye-movement \\
\hline OK: & Oberkiefer \\
\hline OSAS: & obstruktives Schlafapnoesyndrom \\
\hline $\mathrm{p}:$ & Wahrscheinlichkeit \\
\hline PNS: & Spina nasalis posterior \\
\hline PSG: & Polysomnographie \\
\hline REM: & rapid-eye-movement \\
\hline S.: & Seite \\
\hline Stdabw.: & Standardabweichung \\
\hline Tab.: & Tabelle \\
\hline TPW: & Tongue Pharyngeal Wall \\
\hline UK: & Unterkiefer \\
\hline UPPP: & Uvulo-Palato-Pharyngo-Plastik \\
\hline UPW: & Upper Pharyngeal Wall \\
\hline
\end{tabular}




\section{Anhang: Fragebogen}

\section{Fragebogen Schnarchen und Schlafapnoe}

1. Sind Sie bereits von einem Arzt wegen Verdachts auf Schlafapnoe (nächtliche Atemaussetzer) untersucht worden?

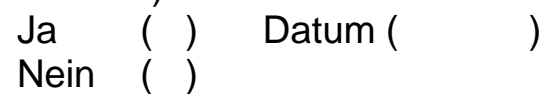

Falls ja, was hat die Untersuchung ergeben?

a) kein Schlafapnoesyndrom

b) bestätigtes Schlafapnoesyndrom

2. Wie groß sind Sie?

Wie viel wiegen Sie?

$\mathrm{cm}$

$\mathrm{kg}$

3. Bitte beantworten Sie die folgenden Fragen zur Schläfrigkeit im Alltag.

Jede Frage können Sie folgendermaßen beantworten:

$\begin{array}{ll}0 & \text { würde nie einnicken } \\ 1 & \text { geringe Wahrscheinlichkeit einzunicken } \\ 2 & \text { mittlere Wahrscheinlichkeit einzunicken } \\ 3 & \text { hohe Wahrscheinlichkeit einzunicken }\end{array}$

Die Situationen sind:

1. Sitzen und lesen

2. Fernsehen

3. als Zuschauer in einer öffentlichen Veranstaltung

(z. B. Theater, Arbeitstreffen)

4. als Mitfahrer in einer einstündigen Autofahrt

5. bei einer Ruhepause nach dem Mittagsessen

6. im Gespräch sitzend mit einer Person

7. nach dem Mittagessen ruhig sitzend (ohne Alkohol)

8. im Auto als Fahrer, wenn der Verkehr für einige Minuten zum stehen kommt

Bitte kreuzen Sie auf der Skala an:

Wie schätzt Ihr Partner die Lautstärke Ihres Schnarchens ein?

Sehr gering

1

$2 \quad 3$

\begin{tabular}{l|l}
3 & 4 \\
\hline
\end{tabular}

5

Wie häufig Schnarchen Sie?

Fast nie

1-2 mal pro Monat

1-2 mal pro Woche

3-4 mal pro Woche

fast jeden Tag

extrem laut

\begin{tabular}{|l|l|l|c|}
\multicolumn{3}{|r}{ extrem laut } \\
\hline
\end{tabular}




\section{Literaturverzeichnis}

Aldrich MS (1989): Automobile Accidents in Patients with Sleep Disorders. Sleep 12: 487494

American Slepp Disorders Association (1992): EEG arousals: scoring rules and example. A Preliminary Report from the Sleep Disorders Atlas Task Force of the American Slepp Disorders Association. Sleep 15: 173-184

American Sleep Disorders Associatiation (1995): Practice Parameters for the Treatment of Snoring and Obstructive Sleep apnea. Sleep 18: 511-513

Andersson L, Brättström V (1991): Cephalometric analysis of permanently snoring patients with and without obstructive sleep apnea syndrome. Int J Oral Maxillofac Surg 20: 159-162

Andreas S, von Breska B, Magnusson K, Kreuzer H (1993): Validation of automated sleep stage and apnoea analysis in suspected sleep apnoea. Eur Respir J $\underline{6}: 48-52$

Arai Y, Tammisalo E, Hashimoto K, Shinoda K (1999): Development of a compact computer tomographic apparatus for dental use. Dentomaxillofac Radiol 28: 245-248

Arzt M, Young T, Finn L, Skatrud JB, Bradley TD (2005): Association of sleep-disordered breathing and the occurrence of stroke. Am J Respir Crit Care Med 172: 1447-1451

Avrahami E, Englender M (1995): Relation between CT Axial Cross-sectional Area of the Oropharynx and Obstructive sleep Apnea Syndrome in Adults. Am J Neuroradiol 16: 135-140

Bacon WH, Turlot JC, Krieger J, Stierle JL (1990): Cephalometric evaluation of pharyngeal obstructive factors in patients with sleep apneas syndrome. Angle Orthod $\underline{60}$ : 115-122

Baguet JP, Barone-Rochette G, Pepin JL (2009): Hypertension and obstructive sleep apnoea syndrome: current perspectives. J Hum Hypertens 23: 431-443 
Baik UB, Suzuki M, Ikeda K, Sugawara J, Mitani H (2002): Relationship between cephalometric characteristics and obstructive sites in obstructive sleep apnea syndrome. Angle Orthod 72: 124-134

Battagel JM, L'Estrange PR (1996): The cephalometric morphology of patients with obstructive sleep apnoea (OSA). Eur J Orthod 18: 557-569

Battagel JM, Johal A, Kotecha B (2000): A cephalometric comparison of subjects with snoring and obstructive sleep apnoea. Eur J Orthod 22: 353-365

Battagel JM, Johal A, Smith AM, Kotecha B (2002): Postural variation in oropharyngeal dimensions in subject with sleep disordered breathing: a cephalometric study. Eur J Orthod 24: $263-276$

Benz C, Sonnabend E, Wilhelm M: Röntgentechnik und -verordnung. 2. Auflage, Urban \& Fischer Verlag München, Jena 2003

Berry DTR, Webb WB, Block AJ (1984): Sleep apnea syndrome: A critical review of the apnea index as diagnostic criterion. Chest $\underline{86}$ : 529-531

Bixler EO, Vgontzas AN, Ten HT, Tyson K, Kales A (1998): Effects of age on sleep apnea in men: I. Prevalence and severity. Am J Respir Crit Care Med 157: 144-148

Bixler EO, Vgontzas AN, Lin HM, Ten Have T, Rein J, Vela-Bueno A, Kales A (2001): Prevalence of sleep-disordered breathing in women: effects of gender. Am J Respir Crit Care Med 163: 608-613

Block AJ, Wynne JW, Boysen PG (1989): Sleep-disordered breathing and nocturnal oxygen desaturation in postmenopausal women.Am J Med 69: 75-79

Bohlman ME, Haponik EF, Smith PL, Allen RP, Bleecker ER, Goldman SM (1983): CT Demonstration of Pharyngeal Narrowing in Adult Obstructive Sleep Apnea. AJR Am J Roentgenol 140: 543-548 
Brown IG, Mc Clean PA, Boucher R, Zamel N, Hoffstein V (1987): Changes in pharyngeal cross-sectional area with posture and application of continuous positve airway pressure in patients with obstructive sleep apnea. Am Rev Respir Dis 136: 628-632

Caballero P, Alvarez-Sala R, Garcia-Rio F, Prados C, Hernán MA, Villamor J, Alvarez-Sala JL (1998): CT in the evaluation of the upper airway in healthy subjects and in patients with obstructive sleep apnea syndrome. Chest $\underline{113}$ : 111-116

Cacaci C, Frank E, Bumann A (2007): DVT-Volumentomograph Digitaler Durchblick. J Cont Dent Educ 3: 1-9

Cirignotta F, Lugaresi E (1980): Some cineradiographic aspects of snoring and obstructive apnea. Sleep 3: 225-226

Cohnen M, Kemper J, Möbes O, Pawelzik J, Mödder U (2002): Radiation dose in dental radiology. Eur Radiol 12: 634-637

Coughlin SR, Mawdsley L, Mugarza JA, Calverley PM, Wilding JP (2004): Obstructive sleep apnoea is independently associated with an increased prevalence of metabolic syndrome. Eur Heart J 25: 735-741

Cuccia AM, Campisi G, Cannavale R, Colella G (2007): Obesity and craniofacial variables in subjects with obstructive sleep apnea syndrome: comparisons of cephalometric values. Head Face Med 3: 41

Danforth RA (2003): Cone beam volume tomography: a new digital imaging option for dentistry. J Calif Dent Assoc 31: 814-815

Dirsch P (2008): Digitale Volumentomografie in der zahnärztlichen Implantologie. ZWP spezial 7+8: 10-14

Dücker J: Praxisleitfaden Zahnärztliche Radiologie. Elsevier GmbH, Urban und Fischer Verlag München 2006 
Duran J, Esnaola S, Rubio R, Iztueta A (2001): Obstructive sleep apnea-hypopnea and related clinical features in a population-based sample of subjects aged 30 to $70 \mathrm{yr}$. Am J Respir Crit Care Med 163: 685-689

Edström KF, Larsson H, Larsson L (1992): Neurogenic effects on the palatopharyngeal muscle in patients with obstructive sleep apnoea: a muscle biopsy study. J Neurol Neurosurg Psychiatry 55: 916-920

Enciso R, Nguyen M, Shigeta Y, Ogawa T and Clark GT (2010): Comparison of cone-beam CT parameters and sleep questionnaires in sleep apnea patients and control subjects. Oral Surg Oral Med Oral Pathol Oral Radiol Endod 109: 285-293

Engelke W, Mendoza M, Repetto G (2006): Preliminary radiographic observations of the tongue-repositioning Manoeuvre. Eur J Orthod 28: 618-623

Fairburn SC, Waite PD, Vilos G, Harding SM, Bernreuter W, Cure J, Cherala S (2007): Three-dimensional changes in upper airways of patients with obstructive sleep apnea following maxillomandibular advancement. J Oral Maxillofac Surg 65: 6-12

Fajdiga I (2005): Snoring imaginga: could Bernoulli explain it all? Chest 128: 896-901

Ferguson KA, Ono T, Lowe AA, Ryan CF, and Fleetham JA (1995): The relationship between obesity and craniofacial structure in obstructive sleep apnea. Chest 108: 375-381

Findley LJ, Unverzagt ME, Suratt PM (1988): Automobile Accidents Involving Patients with Obstructive Sleep Apnea. Am Rev Respir Dis 138: 337-340

Fischer J. (1991): Deutsche Gesellschaft für Pneumologie: Empfehlungen zur Diagnostik und Therapie nächtlicher Atmungs- und Kreislaufregulationsstörungen. Pneumologie 45: 45-48

Flinzberg S, Schmelzle R, Schulze D, Rother U, Heiland M (2003): 3-dimensional imaging possibilities of the mid-face area using digital volume tomography based on a daver study of angle stable osteosynthesis. Mund Kiefer Gesichtschir 7: 289-293 
Fogel RB, Malhotra A, White DP (2004): Sleep 2: Pathophysiology of obstructive sleep apnea/hypopnea syndrome. Thorax 59: 159-163

Fuhrmann A, Schulze D, Rother U, Vesper M (2003): Digital transversal slice imaging in dental-maxillofacial radiology: from pantomography to digital volume tomography. Int $\mathbf{J}$ Comput Dent ㅁ: 129-140

Fujita S, Conway W, Zorick F, Roth T (1981): Surgical correction of anatomic abnormalities in obstructive sleep apnea syndrome: Uvulopalatopharyngoplasty. Otolaryngol Head Neck Surg 89: 923-934

Galvin JR, Rooholamini SA, Stanford W (1989): Obstructive Sleep Apnea: Diagnosis with Ultrafast CT. Radiology 171: 775-778

Gastaut H, Tassinari CA, Duron B (1966): Polygraphic study of the episodic diurinal and nocturnal (hypnic and respiratory) manifestations of the Pickwick Syndrome. Brain Res 2: $167-186$

Gislason T, Almquist M, Erikson G, Taube A, Boman G (1988): Prevalence of sleep apnea syndrome among Swedish men - an epidemiological study. J Clin Epidemiol 41: 571-576

Gleeson K, Zwillich C, White DP (1990): The influence of increasing ventilatory effort on arousal from sleep. Am Rev Respir Dis 142: 295-300

Goch I: Vergleich metrischer Genauigkeit von Computertomographie, digitaler Volumentomographie und Orthopantomographie in der modernen Implantologie. Med. Diss. Düsseldorf 2005

Gould GA, Whyte KF, Rhind GB, Airlie MA, Catterall JR, Shapiro CM, Douglas NJ (1988): The sleep hypopnea syndrome. Am Rev Respir Dis 137: 895-898

Grassino AE, Begin P (1990): The role of respiratory muscles in the interruption of the obstructive sleep apnea. Rev Mal Respir 7: 403-410 
Guilleminault C, Tilkian A, Dement WC (1976): The sleep apnea syndromes. Annu Rev Med 27: 465-484

Guilleminault C, Hill MW, Simmons FB, Dement WC (1978): Obstructive sleep apnea: electromyographic and fiberoptic studies. Exp Neurol 62: 48-67

Guilleminault C, Stoohs R, Clerk A, Cetel M, Maistros P (1993): A cause of excessive daytime sleepiness. The upper airway resistance syndrome. Chest 104: 781-787

Guilleminault C, Kushida C, Stoohs R, Ohayon M, Wilson K, Clerk A (1996): Should everyone be monitored for upper-airway resistance and how? Sleep 19: 260-262

Hader C, Hinz M, Welz-Barth A, Rasche K (2006): Sleep disordered breathing in the elderly: a three year longitudinal cohort study. J Physiol Pharmacol 57: 119-129

Haponik EF, Smith PL, Bohlman ME, Allen RP, Goldman SM, Bleecker ER (1983): Computerized tomographie in obstructive sleep apnea. Am Rev Respir Dis 127: 221-226

Harmon JD, Morgan W, Chaudhary B (1989): Sleep apnea: morbidity and mortality of surgical treatment. South Med J $\underline{82}$ : 161-164

Harston LE, Sauerland EK (1981): Elektromyography of the human palate: Discharge patterns of the levator and tensor veli palatini. Electromyogr Clin Neurophysiol 21: 287-297 Hatcher DC, Aboudara CL (2004): Diagnosis goes digital. Am J Orthod Dentofacial Orthop 125: $512-5$

Herold G: Innere Medizin. Gerd Herold Eigenverlag Köln 2000

Heurich T, Ziegler C, Steveling H, Wörtche R, Mühling J, Hassfeld S (2002): Digital volume tomography - an extension to the diagnostic procedures available for application before surgical removal of third molars. Mund Kiefer Gesichtschir ㅁ: 427-432 
Hillman DR, Platt PR und Eastwood PR (2003): The upper airway during anaesthesia. Br J Anaesth 91: 31-39

Hochban W, Brandenburg U, Schürmann R (1994): Zur Gesichtsskelettmorphologie beim obstruktiven Schlafapnoe-Syndrom und ihrem Einfluß auf die Atemwegsobstruktion. Dtsch Zahnärztl Z $\underline{49}$ : 777-782

Hochban W, Brandenburg U, Peter JH (1995): Surgical treatment of obstructive sleep apnea by osteotomy of the facial bones (results of 1 year). Pneumologie 49: 175-179

Hoffstein V, Slutsky AS (1987): Pharyngeal structure and function as a determinant of sleeprelated breathing disorders: a unifying hypothesis. Med Hypotheses 24: 191-199

Holberg C, Steinhäuser S, Geis P, Rudzki-Janson I (2005): Cone-Beam Computed Tomography in Orthodontics: Benefits and Limitations. J Orofac Orthop 66: 434-444

Hollowell DE, Suratt PM (1991): Mandible position and activation of submental and masseter muscles during sleep. J Appl Physiol 71: 2267-2273

Hora F, Nápolis LM, Daltro C, Kodaira SK, Tufik S, Togeiro SM, Nery LE (2007): Clinical, Anthropometric and Upper Airway Anatomic Characteristics of Obese Patients with Obstructive Sleep Apnea Syndrome. Respiration 74: 517-524

Horner RL, Mohiaddin RH, Lowell DG, Shea SA, Burman ED, Longmore DB, Guz A (1989): Sites and sizes of fat deposits around he pharynx in obese patients with obstructive sleep apnea and weight matches controls. Eur Respir J 2: 613-622

Horner RL, Innes JA, Murphy K, Guz A (1991): Evidence for reflex upper airway dilator muscle activation by sudden negative airway pressure in man. J Physiol $\underline{436}$ : $15-29$

Houston WJB (1983): The analysis of errors in orthodontic measurements. Am J Orthod $\underline{83}$ : $382-390$ 
http://www.orangedental.de/index.php/3d-digitales-roentgen/pax-reve3d (1.11.10)

http://de.wikipedia.org/wiki/Polysomnographie (24.1.11)

http://www.innovations-report.de/html/berichte/studien/zungenimplantat_gegen_ naechtliche_atemaussetzer_118043.html (24.1.11)

Hübinger A: Evaluation dreidimensionaler radiologischer Verfahren zur Darstellung der Mandibula am Humanpräparat. Med. Diss. Gießen 2008

Hümmeke S, Esser E, Vosshans J (2003): Diagnostische Möglichkeiten der digitalen Volumentomographie (DVT). Quintessenz 54: 1105-1112

Hultcrantz E, Svanholm H (1991): Down syndrome and sleep apnea - a therapeutic challenge. Int J Pediatr Otorhinolaryngol 21: 263-268

Ingbar DH, Gee JBL (1985): Pathopysiology and Treatment of Sleep Apnea. Annu Rev Med 36: 369-395

Ingman T, Nieminen T, Hurmerinta K (2004): Cephalometric comparison of pharyngeal changes in subjects with upper airway resistance syndrome or obstructive sleep apnoea in upright and supine positions. Eur J Orthod 26: 321-326

Ip MS, Lam B, Ng MM, Lam WK, Tsang KW, Lam KS (2002): Obstructive sleep apnea is independently associated with insulin resistance. Am J Respir Crit Care Med 165: 670-676

Issa FG, Sullivan CE (1984): Upper airway closing pressures in obstructive sleep apnea. J Appl Physiol 57: 520-527

Johal A, Conaghan C (2004): Maxillary morphology in obstructive sleep apnea: a cephlometric an model study. Angle Orthod 74: 648-656

Johal A, Patel SI, Battagel JM (2007): The relationship between craniofacial anatomy and obstructive sleep apnoea: a case-controlled study. J Sleep Res 16: 319-326 
Johns MW (1993): Daytime Sleepiness, Snoring and Obstructive Sleep Apnea.Chest 103: 3036

Jordan AS, McEvoy RD (2003): Gender differences in sleep apnea: epidemiology, clinical presentation and pathogenic mechanisms. Sleep Med Rev 7: 377-389

Kapur V, Blough DK, Sandblom RE, Hert R, de Maine JB, Sullivan SD, Psaty BM (1999): The Medical Cost of Undiagnosed Sleep Apnea. Sleep 22: 749-755

Kingshott RN, Cowan JO, Jones DR, Flannery EM, Smith AD, Herbison GP, Taylor DR (2004): The role of sleep-disordered breathing, daytime sleepiness, and impaired performance in motor vehicle crashes-a case control study. Sleep Breath $\underline{8}$ : 61-72

Kim TH, Chun BS, Lee HW, Kim JS (2010): Differences of Upper Airway Morphologhy According to Obesity: Study with Cephalometry and Dynamic MD-CT. Clin Exp Otorhinolaryngol 3: 147-152

Kistler F (2007): Sichere Implantatplanung dank 3D-Diagnostik. Dent Implantol 11: 454-456

Koren A, Gronelj LD, Fajdiga I (2009): CT comparison of primary snoring and obstructive sleep apnea syndrome: role of pharyngeal narrowing ratio and soft palate-tongue contact in awake patient. Eur Arch Otorhinolaryngol 266: 727-734

Lamberg L (2007): Menopause not always to blame for sleep problems in midlife women. JAMA 297: 1865-1866

Lavie P (1983): Incidence of sleep apnea in a presumably healthy working population: A signifikant relationship with excessive daytime sleepiness. Sleep $\underline{6}$ : 312-318

Langwieder K, Sporner A, Hell W: Struktur der Unfälle mit Getöteten auf Autobahnen im Freistaat Bayern im Jahr 1991 HUK-Verband, Büro für Kfz-Technik, München 1994

Leger D (1994): The Cost of Sleep-Related Accidents: A Report for the National Commission on Sleep Disorders Research. Sleep 17: 84-93 
Lenza MG, Lenza de O MM, Dalstra M, Melsen B, Cattaneo PM (2010): An analysis of different approaches to the assesment of upper airway morphology: a CBCT study. Orthod Craniofac Res 13: 96-105

Li S, Shi H, Qü S, Hua Z, Dong X, Dong W (2002): The lingual region upper airway CT scan of obstructive sleep apnea patients. Zhonghua Kou Qiang Yi Xue Za Zhi 37: 415-417

Lindberg E, Gislason T (2000): Epidemiology of sleep-related obstructive breathing. Sleep Med Rev 4: 411-433

Lojander J, Mustijoki P, Ronka S, Mecklin P, Maaslita P (1998): A nurse-managed weight reduction programme for obstructive sleep apnea syndrome. J Intern Med 244: 251-255

Lomoschitz F, Schima W, Schober E, Pokieser P, Youssefzadeh S, Kainberger F, Czerny C, Imhof H (2000): The pharynx. The imaging of its normal anatomy. Radiologe 40: 601-609

Lowe AA, Takada K, Yamagata Y, Sakuda M (1985): Dentoskeletal and tongue soft-tissue correlates: A cephalometric analysis of rest postion. Am J Orthod 로: 333-341

Lowe AA, Santamaria JD, Fleetham JA, Price C (1986): Facial morphology and obstructive sleep apnea. Am J Orthod Dentofacial Orthop 90: 484-491

Lowe AA, Fleetham J, Ryan F, Mathews B (1990): Effects of a mandibular repositioning appliance used in the treatment of obstructive sleep apnea on tongue muscle activity. Prog Clin Biol Res 345: 395-404

Lugaresi E, Cirignotta F, Coccagna G, Piana C (1980): Some epidemiological data on snoring and cardiocirculatory disturbances. Sleep $\underline{3}$ : 221-224

Mayer-Brix J, Glanz H, Schulze W, Meier-Ewert K (1988): HNO-ärztliche Befunde bei obstruktivem Schlaf-Apnoe-Syndrom. HNO 36: 133-139

Miki H, Hida W, Kikuchi Y, Takishima T (1988): Effect of sleep position on obstructive sleep apnea. Tohoku J Exp Med 156: 143-149 
Möbes O, Becker J, Pawelzik J, Jacobs K (1999): Anwendungsmöglichkeiten der digitalen Volumentomographie in der implantologischen Diagnostik. Z Zahnärztl Implantol 15: 229233

Möbes O, Becker J, Schnelle C, Ewen K, Kemper J, Cohnen M (2000): Strahlenexposition bei der digitalen Volumentomographie, Panoramaschichtaufnahme und Computertomographie. DZZ ㅍ: 336-339

Mortimore IL, Kochhar P, Douglas NJ (1996): Effect of chronic continuous positive airway pressure (CPAP) therapy on upper airway size in patients with sleep apnoea/hypopnoea syndrome. Thorax 51: 190-192

Mozzo P, Procacci C, Tacconi A, Martini P, Amdreis IA (1998): A new volumetric CT machine for dental imaging based on the cone beam technique: preliminary results. Eur Radiol 8: 1558-1564

Noda A, Yagi T, Yokota M, Kayukawa Y, Ohta T, Okada T (1998 a): Daytime sleepness and automobile accidents in patients with obstructive sleep apnea syndrome. Psychiatry Clin Neurosci 52: 221-222

Noda A, Okada T, Yasuma F, Sobue T, Nakashima N, Yokota M (1998 b): Prognosis of the middle-aged and aged patients with obstructive sleep apnea syndrome. Psychiatry Clin Neurosci 52: 79-85

Onat A, Hergenc G, Uyarel H, Yazici M, Tuncer M, Dogan Y, Can G, Rasche K (2007): Obstructive sleep apnea syndrome is associated with metabolic syndrome rather than insulin resistance. Sleep Breath 11: 23-30

Oosterkamp BC, Remmelink HJ, Pruim GJ, Hoekema A, Dijkstra PU (2007): Craniofacial, craniocervical, and pharyngeal morphology in bilateral cleft lip and palate and obstructive sleep apnea patients. Cleft Palate Craniofac J $\underline{44}$ : 1-7

Pack AI, Pien GW (2004): How much do crashes related to obstructive sleep apnea cost? Sleep 27: 369-370 
Partinen M, Guilleminault C, Quera-Salva MA, Jamieson A (1988): Obstructive Sleep Apnea and Cephalometric Roentgenograms. Chest 93: 1199-1205

Partinen M, Telakivi T (1992): Epidemiology of obstructive sleep apnea syndrome. Sleep $\underline{15}$ : $1-4$

Pasler F, Visser H: Farbatlanten der Zahnmedizin; Band 5: Zahnmedizinische RadiologieBildgebende Verfahren; 2. überarbeitete Auflage; Thieme Verlag Stuttgart. 2000

Pepin JL, Ferretti G, Veale D, Romand P, Coulomb M, Brambilla C, Lévy PA (1992):

Somnofluoroscopy, computed tomography, and cephalometry in the assessment of the airway in obstructive sleep apnoea. Thorax 47: 150-156

Pepin JLD, Veale D, Ferretti GR, Mayer P, Levy PA (1999): Obstructive Sleep Apnea Syndrome: Hooked Appearance of the Soft Palate in Awake Patients-Cephalometric and CT Findings. Radiology 210: 163-170

Peppard PE, Young T, Palta M, Skatrud J (2000): Prospective study of the association between sleepdisordered breathing and hypertension. N Engl J Med 342: 1378-1384

Peter JH, Faust M, Fett I, Podszus T, Schneider H, Weber K, von Wichert P (1990): Die Schlafapnoe. Dtsch Med Wochenschr 115: 182-186

Peter JH, Becker H, Blanke J, Clarenbach P, Mayer G, Raschke F, Rühle KH, Rüther E, Schläfke M, Schönbrunn E (1991): Empfehlungen zur Diagnostik, Therapie und Langzeitbetreuung von Patienten mit Schlafapnoe. Med Klin $\underline{86}$ : 46-50

Peter JH, Blanke J, Cassel W, Clarenbach P, Elek H, Faust M, Fietze I, Lund R, Mahlo HW, Mayer G (1992): Empfehlungen zur ambulanten Diagnostik der Schlafapnoe. Med Klin 87: $310-317$

Peter JH, Koehler U, Grote L, Podszus T (1995): Manifestations and consequences of obstructive sleep apnea. Eur Respir J $\underline{8}$ : 1572-1583 
Plywaczewski R, Bednarek M, Jonczak L, Zielinski J (2007): Sleep-disordered breathing in a middle-aged and older Polish urban population. J Sleep Res 17: 73-81

Podszus T (1993): Empfehlungen zur nächtlichen nasalen Beatmungstherapie bei Atmungsstörungen. Pneumologie 47: 333-335

Polo OJ, Tafti M, Fraga J, Porkka KV, Dejean Y, Billiard M (1991): Why don't all heavy snorers have obstructive sleep apnea? Am Rev Respir Dis 143: 1288-1293

Pracharktam N, Nelson S, Hans MG, Broadbent BH, Redline S, Rosenberg C, Strohl KP (1996): Cephalometric assessment in obstructive sleep apnea. Am J Orthod Dentofacial Orthop 109: 410-419

Pradel W, Schmidt F, Paditz E, Eckelt U (2000): Stellenwert der Radiokephalometrie in der Diagnostik bei OSAS im Erwachsenalter. Somnologie 4: 96-100

Punjabi NM, Polotsky VY (2005): Disorders of glucose metabolism in sleep apnea. J Appl Physiol 99: 1998-2007

Punjabi NM, Shahar E, Redline S, Gottlieb DJ, Givelber R, Resnick HE (2004):

Sleepdisordered breathing, glucose intolerance, and insulin resistance: the Sleep Heart Health Study. Am J Epidemiol 160: 521-530

Rasche K, Sanner B, Schäfer T: Schlafbezogene Atmungsstörungen in Klinik und Praxis. Blackwell Wissenschafts-Verlag Berlin, Wien 1999

Remmers JE, deGroot WJ, Sauerland EK, Anch AM (1978): Pathogenesis of upper airway occlusion during sleep. J Appl Physiol 44: 931-938

Riha RL, Brander P, Vennelle M, Douglas NJ (2005): A cephalometric comparison of patients with the sleep apnea/hypopnea syndrome and their siblings. Sleep 28: 315-320

Riley R, Guilleminault C, Herran J, Powell N (1983): Cephalometric Analyses and FlowVolume Loops in Obstructive Sleep Apnea Patients. Sleep ㅁ: 303-311 
Riley R, Powell N, Guilleminault C (1986): Cephalometric roentgenograms and computerized tomographic scans in obstructive sleep apnea. Sleep 9: 514-515

Rodenstein DO (1992): Assessment of uvulopalatopharyngoplasty for the treatment of sleep apnea syndrome. Sleep $\underline{15}$ : 56-62

Rother UJ: Moderne bildgebende Diagnostik in der Zahn-, Mund- und Kieferheilkunde. 2. Auflage. Elsevier GmbH Müchen. 2006

Ryan CM, Bradley TD (2005): Pathogenesis of obstructive sleep apnea. J Appl Physiol 99: $2440-2450$

Sackner MA, Landa J, Forrest T, Greeneltch D (1975): Periodic sleep apnea: chronic sleep deprivation related to intermittent upper airway obstruction and central nervous system disturbance. Chest 67: 164-171

Sakakibara H, Tong M, Matsushita K, Hirata M, Konishi Y, Suetsugu S (1999):

Cephalometric abnormalities in non-obese and obese patients with obstructive sleep apnoea. Eur Respir J 13: 403-410

Scarfe WC, Farman AG, Sukovic P (2006): Clinical applications of conebeam computed tomography in dental practice. J Can Dent Assoc 72: 75-80

Schäfer J: Schnarchen, Schlafapnoe und obere Luftwege. Georg Thieme Verlag 1996

Schäfer J, Lenders H (1990): Anamnese und Polysomnographie bei Patienten mit Rhonchopathien und obstruktiven Apnoe-Syndrom: Ein Datenvergleich bei 140 Patienten. Laryngorhinootologie 69: 595- 599

Scherer P, Neugebauer J, Ritter L, Mischkowski R, Scheer M, Zö.ller JE (2007): Indikationen für die 3-dimensionale Bildgebung in der Zahnheilkunde. ZWR - Das deutsche Zahnärzteblatt 116: $219-230$ 
Schmidt RF, Lang F, Thews G: Physiologie des Menschen 29. Auflage. Springer Medizin Verlag Heidelberg. 2005

Schmidt-Nowara WW, Meade TE, Hays MB (1991): Treatment of snoring and obstructive sleep apnea with a dental orthosis. Chest 99: 1378-1385

Schnelle C : Vergleich der Strahlenexposition bei der Digitalen-Volumen-Tomographie, der Panoramaschichtaufnahme und der Computertomographie. Med. Diss. Düsseldorf 2001

Schwab RJ, Gupta KB, Gefter WB, Metzger LJ, Hoffman EA, Pack AI (1995): Upper airway and soft tissue anatomy in normal subjects and paitens with sleep-disordered breathing. Significance of the lateral pharyngeal walls. Am J Respir Crit Care Med 152: 1673-1689

Schwenzer N, Ehrenfeld M: Zahn-Mund-Kiefer-Heilkunde, Band 1 Allgemeine Chirurgie 3. Auflage Georg Thieme Verlag Stuttgart, New York 2000

Shahar E, Whitney CW, Redline S, Lee ET, Newman AB, Javier Nieto F, O'Connor GT, Boland LL, Schwartz JE, Samet JM (2001): Sleep-disordered breathing and cardiovascular disease: cross-sectional results of the Sleep Heart Health Study. Am J Respir Crit Care Med 163: $19-25$

Shen H (1993): Pharyngeal cross-sectional area and pharyngeal compliance in normal Japanese males and females. Nihon Kyobu Shikkan Gakkai Zasshi 31: 1432-1440

Shepard JW Jr. (1992): Hypertension, cardiac arrythmias, myocardial infarction and stroke in relation to obstructive sleep apnea. Clin Chest Med 13: 437-458

Shepard JW Jr., Thawley SE (1990): Localization of upper airway collaps during sleep in patients with obstructive sleep apnea. Am Rev Respir Dis 141: 1350-1355

Siegrist J, Peter J, Himmelmann J, Geyer S (1987): Fragebogen zum Schlafverhalten. Prax Klin Pneumol 41: 357-363 
Silbernagel S, Pape HC, Klinke R: Physiologie 5. Auflage. Georg Thieme Verlag Stuttgart 2005

Soler M, Tamm M (1995): Das obstruktive Schalfapnoesyndrom. Schweiz Med Wochenschr 125: $1007-1014$

Solow B, Skov S, Ovesen J, Norup PW, Wildschiodtz G (1996): Airway dimensions and head posture in obstructive sleep apnoea. Eur J Orthod 18: 571-579

Stratemann SA, Huang JC, Maki K, Miller AJ, Hatcher DC (2008): Comparison of cone beam computed tomography imaging with physical measures. Dentomaxillofac Radiol 37: 80-93

Strollo PJ Jr., Rogers RM (1996): Obstructive sleep apnea. N Engl J Med 334: 99-104

Sukerman S, Healy GB (1979): Sleep apnea syndrome associated with upper airway obstruction. Laryngoscope 89: 878-885

Sullivan CE, Issa FG, Berthon- Jones M, Eves L (1981): Reversal of obstructive sleep apnea by continuous positive airway pressure applied through the nares. Lancet 1981, 1: 862- 865

Sullivan CE, Issa FG, Berthon-Jones M, McCauley VB, Costas LJ (1984): Home treatment of obstructive sleep apnea with continuous positive airway pressure applied through a nosemask. Bull Eur Physiopathol Respir 20: 49-54

Suomalainen A, Vehmas T, Kortesniemi M, Robinson S, Peltola J (2008): Accuracy of linear measurements using dental cone beam and conventional multislice computed tomography. Dentomaxillofac Radiol 37: 10-17

Tangel DJ, Mezzanotte WS, White DP (1991): Influence of sleep on tensor palatini EMG and upper airway resistance in normal men. J Appl Physiol 70: 2574-2581

Tangugsorn V, Skatvedt O, Krogstad O, Lyberg T (1995): Obstructive sleep apnoea: a cephalometric study. Part II. Uvulo-glossopharyngeal morphology. Eur J Orthod 17: 57-67 
Teitelbaum J, Diminutto M, Comiti S, Pépin JL, Deschaux C, Raphaël B, Bettega G (2007): Lateral cephalometric radiography of the upper airways for evaluation of surgical treatment of obstructive sleep apnea syndrome. Rev Stomatol Chir Maxillofac 108: 13-20

Thiel HJ, Hassfeld S: Cone-Beam CT: Digitale Volumentomographie für den Zahn- und Kieferbereich, in Schnittbilddiagnostik in der MKG Medizin und Zahnmedizin. Georg Thieme Verlag Stuttgart 2001

Verse T, Bodlaj R, de la Chaux R, Dreher A, Heiser C, Herzog M, Hohenhorst W, Hörmann K, Kaschke O, Kühnel T, Mahl N, Maurer JT, Pirsig W, Rohde K, Sauter A, Schedler M, Siegert R, Steffen A, Stuck BA (2009): Guideline: Treatment of obstructive sleep apnea in adults. HNO $\underline{57}$ : $1136-1156$

Vos W, De Backer J, Devolder A, Vanderveken O, Verhulst S, Salgado R, Germonpre P, Partoens B, Wuyts F, Parizel P, De Backer W (2007): Correlation between severity of sleep apnea and upper airway morphology based on advanced anatomical and functional imaging. $\mathbf{J}$ Biomech 40: 2207-2213

Voßhans J (2005): The anatomical landmarks of bone and teeth with the digital volume tomographie. ZM 95: 32-36

Walker EB, Frith RW, Harding DA, Cant BR (1989): Uvulopalatopharyngoplasty in severe idiopathic obstructive sleep apnea syndrome. Thorax 44: 205-208

Wessendorf TE, Teschler H, Wang YM, Konietzko N, Thilmann AF (2000): Sleep-disordered breathing among patients with first-ever stroke. J Neurol 247: 41-47

Wilcox I, Grunstein RR, Hedner JA, Doyle J, Collins FL, Fletcher PJ, Kelly DT, Sullivan CE (1993): Effect of Nasal Continuous Positive Airway Pressure During Sleep on 24-hour Blood Pressure in Obstructive Sleep Apnea. Sleep 16: 539-544

Wiltfang J, Merten HA, Luhr HG (1996): The functional palatoraphy in the treatment of obstructive sleep apnoea. Br J Oral Maxillofac Surg 34: 82-86 
Wolk R, Kara T, Somers VK (2003): Sleep-disordered breathing and cardiovascular disease. Circulation 108: 9-12

Yaggi HK, Concato J, Kernan WN, Lichtman JH, Brass LM, Mohsenin V (2005): Obstructive sleep apnea as a risk factor for stroke and death. N Engl J Med 353: 2034-2041

Young T, Palta M, Dempsey J, Skatrud J, Weber S, Badr S (1993): The occurence of sleepdisordered breathing among middle-aged adults. N Engl J Med 328: 1230-1235

Young T, Peppard PE, Gottlieb DJ (2002): Epidemiology of obstructive sleep apnea: a population health perspective. Am J Respir Crit Care Med 165: 1217-1239

Young T, Finn L, Austin D, Peterson A (2003): Menopausal status and SDB in the Wisconsin Sleep Cohort Study. Am J Respir Crit Care Med 167: 1181-1185

Yucel A, Unlu M, Haktanir A, Acar M, Fidan F (2005): Evaluation of the Upper Airway Cross-sectional Area Changes in Different Degrees of Severity of Obstructive Sleep Apnea Syndrome: Cephalometric and Dynamic CT Study. AJNR Am J Neuroradiol 26: 2624-2629 


\section{Danksagung}

Mein Dank gilt Herrn Prof. Dr. med. Dr. med. dent. W. Engelke für die gute Betreuung und Zusammenarbeit und für seine zahlreichen Anregungen während der Entstehung dieser Arbeit. Bei Herrn Dr. B. Kirchner möchte ich mich für seine Hilfsbereitschaft bei der technischen Umsetzung am DVT bedanken. Vielen Dank auch an Christoph Schlick für seine Unterstützung in Form von Diskussionen und Vorschlägen. 
